The impact of chest compressions on defibrillation success during out-of-hospital cardiac arrest and haemodynamics in an experimental animal model

Thesis for the degree PhD

Cand. med. Mikkel Torp Steinberg

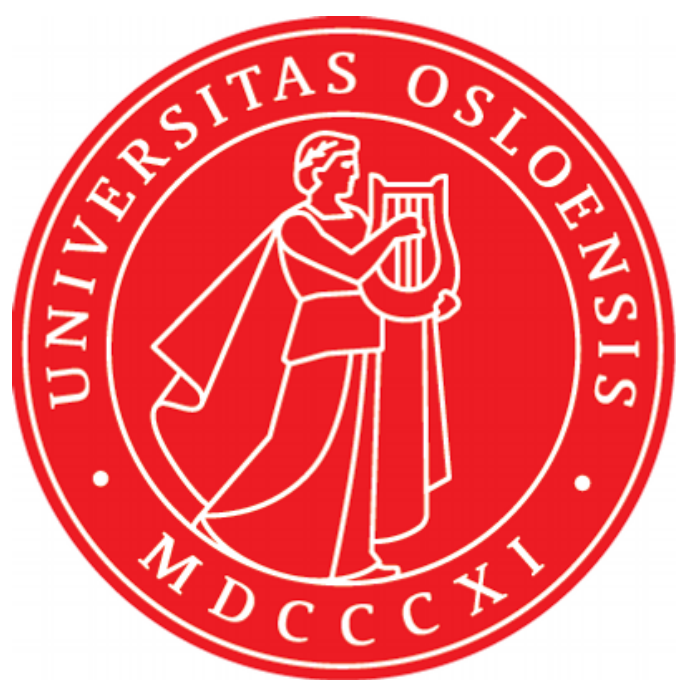

Norwegian National Advisory Unit on Prehospital Emergency Medicine,

Department for Research and Development,

Division of Emergencies and Critical Care,

Oslo University Hospital

$\&$

Institute of Clinical Medicine,

Faculty of Medicine,

University of Oslo 
(C) Mikkel Torp Steinberg, 2018

Series of dissertations submitted to the Faculty of Medicine, University of Oslo

\section{ISBN 978-82-8377-345-3}

All rights reserved. No part of this publication may be reproduced or transmitted, in any form or by any means, without permission.

Cover: Hanne Baadsgaard Utigard.

Print production: Reprosentralen, University of Oslo. 


\section{Table of Contents}

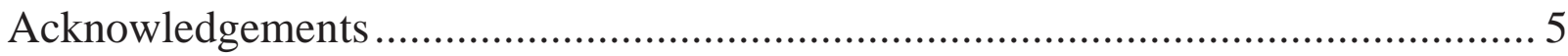

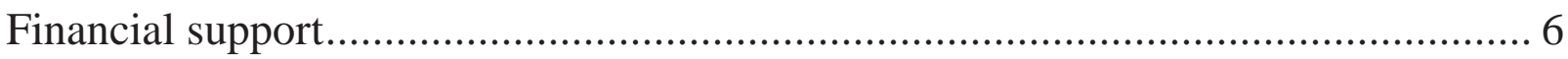

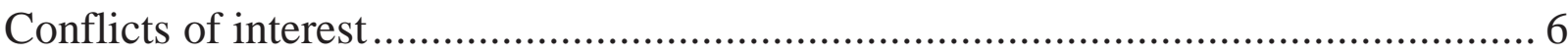

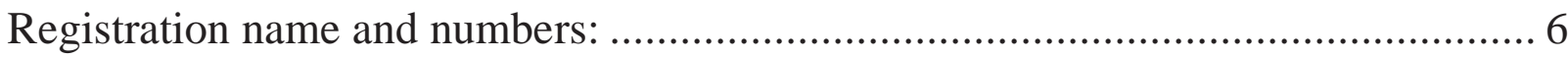

Abbreviations and Glossary ………................................................................... 7

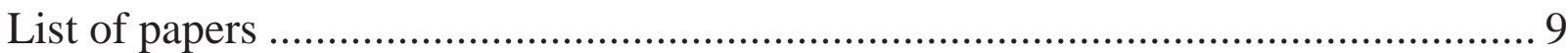

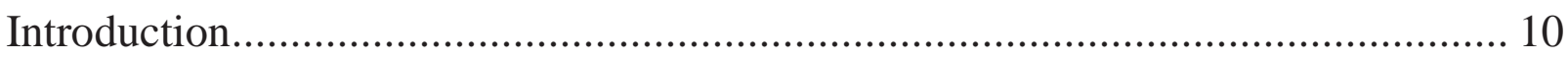

The physiology and haemodynamics behind chest compressions .................................. 11

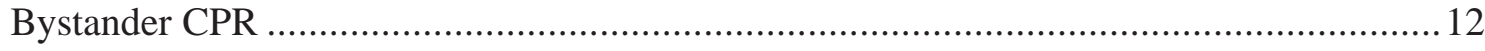

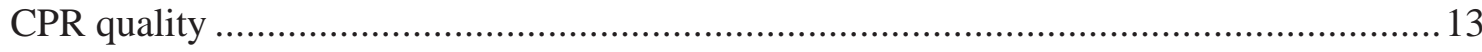

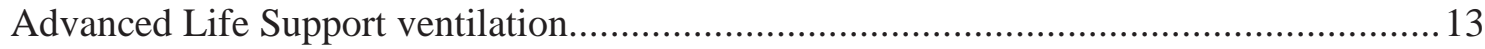

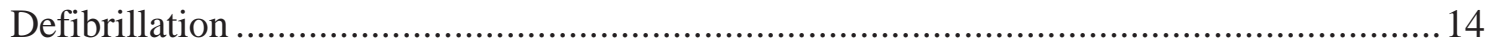

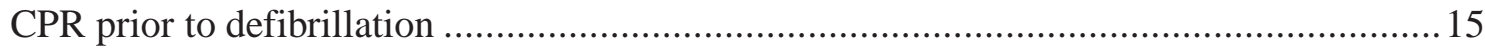

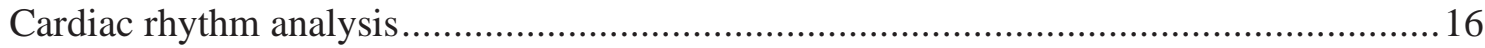

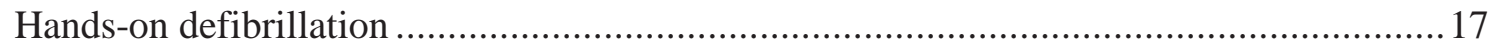

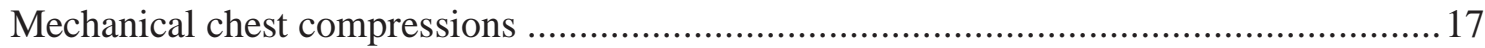

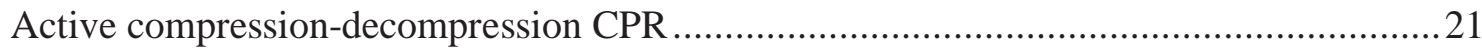

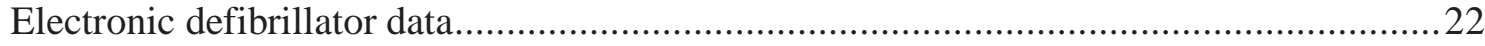

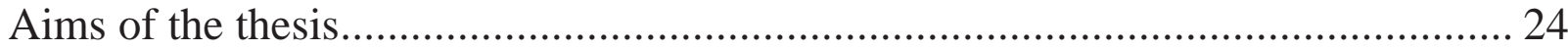

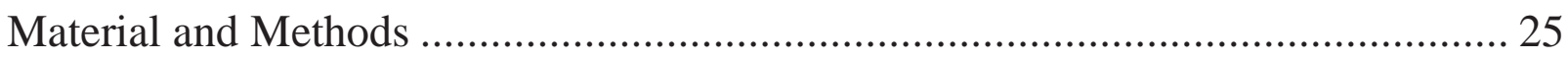

The Circulation Improving Resuscitation Care trial - CIRC ..........................................25

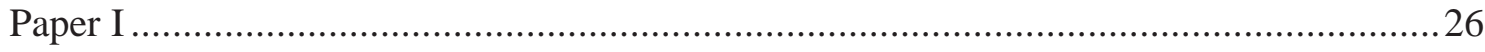

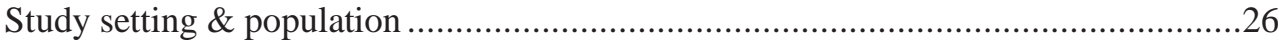

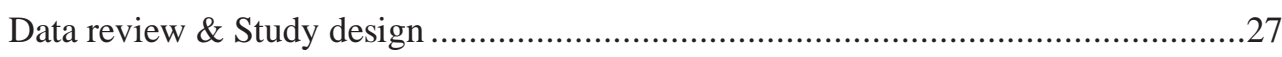

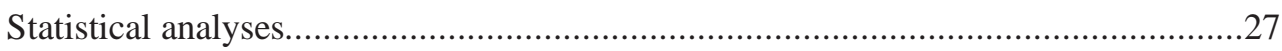

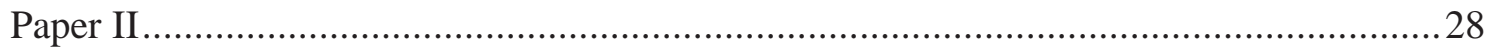

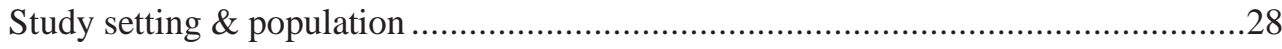

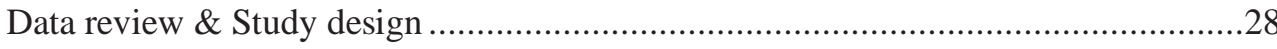

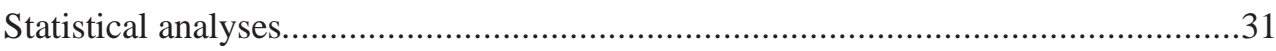

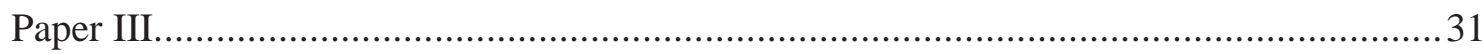




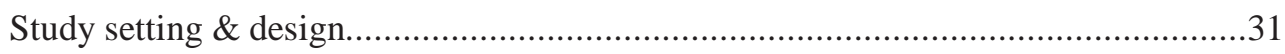

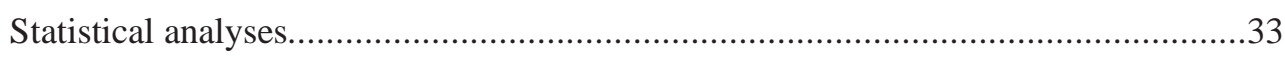

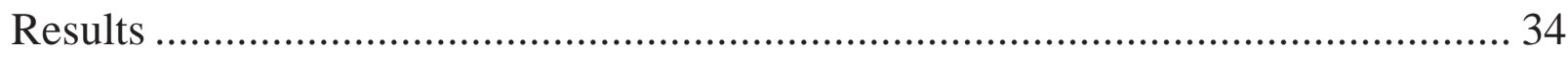

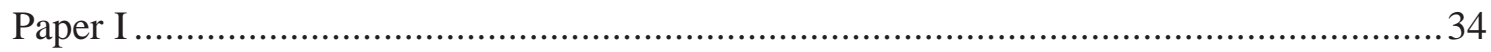

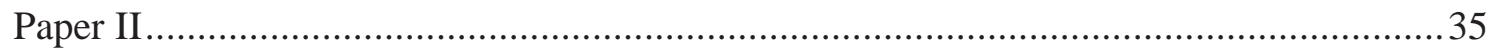

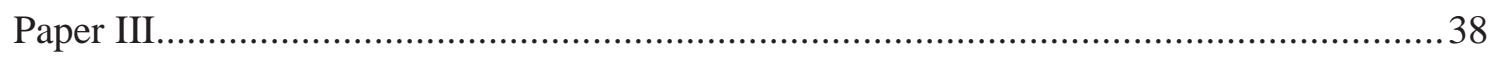

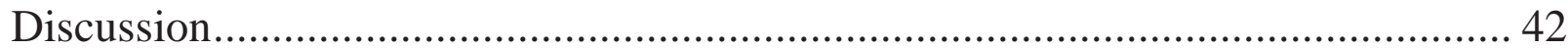

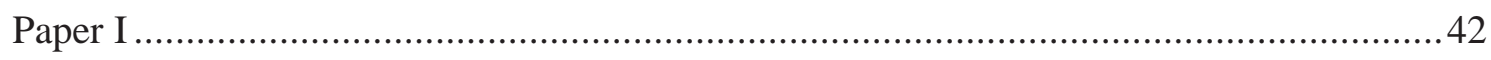

Chest compression pauses and inappropriate shocks ..............................................42

Rhythm conversion without defibrillation ...........................................................44

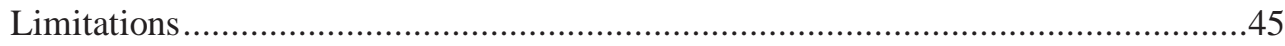

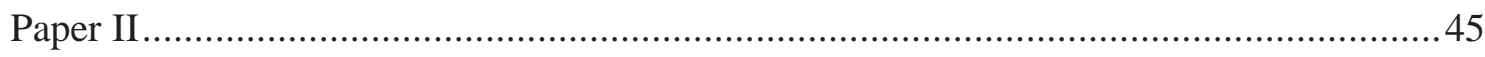

Defibrillation success during chest compressions...................................................45

Why defibrillation during chest compression yields different results? .......................46

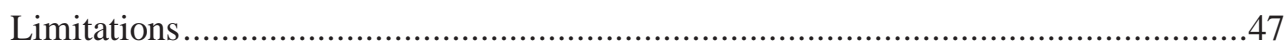

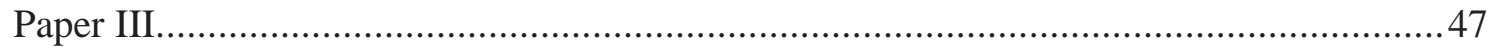

Haemodynamic benefits of ACD-CPR.................................................................

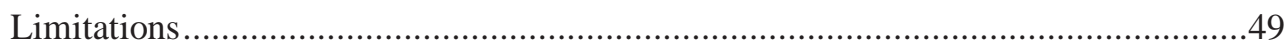

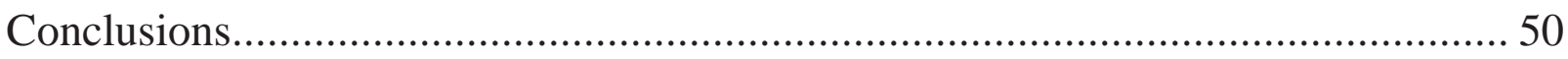

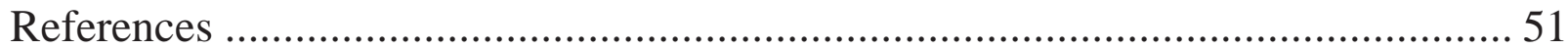

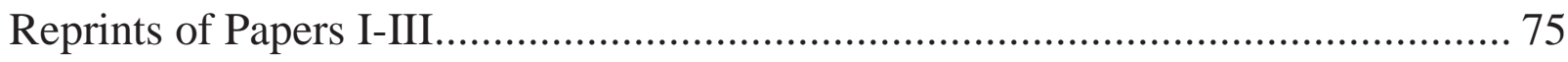




\section{Acknowledgements}

I met Lars Wik on a ride along with the Oslo ambulance service in my second year of medical school. I was early fascinated by emergency medicine, but it was Lars who opened my eyes for research in the same field. What started out as work on a mandatory written assignment in medical school, ended up with me joining the medical student research program for full time emergency medicine research, with Lars as my supervisor. He has since then been a great inspiration and of invaluable help to me in my research in his field of expertise, I can't thank him enough for that. I have also been lucky to have such an esteemed and knowledgeable cosupervisor as professor emeritus Petter Andreas Steen. I thank him especially for his thorough and insightful help in the writing process of all three of my papers and this thesis. I thank my second co-supervisor professor Kjetil Sunde for his thorough help to revise and finish this thesis. I thank all members of the emergency medicine research group at Oslo University Hospital for their guidance and support. Thanks to Jan-Erik Nilsen and everyone at the Norwegian advisory unit on prehospital emergency medicine (NAKOS) where I have had my desk and for all administrational support, insightful lunch discussions, and for supporting my attendance at international conferences. Especially thanks to former PhD candidate Jan-Åge Olsen whom I have collaborated with from day one and who has been of great help teaching me how to work with and analyse electronic defibrillator data. I thank the medical student research program at the University of Oslo for the opportunity to conduct research during my medical studies and for the courses, conferences and presentations I have been lucky to attend. I would also like to thank Oslo University Hospital and the University of Oslo for making it possible for me to continue with my research while undertaking my clinical internship. Thanks to the research support group at Oslo University Hospital, especially Cathrine Brunborg for all the help with statistical analyses. A big thank you to all my co-authors who have given me insightful feedback on all manuscripts. I also want to acknowledge all EMS providers, data collectors, coordinators and monitors who made the CIRC trial possible. I thank my family and friends and especially May-Linn for your constant support of my work.

Oslo, June 2018

Mikkel Torp Steinberg 


\section{Financial support}

ZOLL Medical funded the CIRC trial. Steinberg received an unrestricted research scholarship provided by the Norwegian Research Council, 2013-2015. Olsen was partly funded by an unrestricted grant from Norwegian Health Region South-East and partly by a research grant from ZOLL Medical to NAKOS. All authors' institutions received funding from ZOLL Medical for their participation in the CIRC trial. The open access publication fee for paper III was granted by the University of Oslo publication fund.

\section{Conflicts of interest}

Steinberg, Kramer-Johansen, Neset, Eriksen and Norseng have no conflicts of interest to report. Wik is NAKOS representative in Physio-Control medical advisory board, was principal investigator for the CIRC and LUCAS ACD trials, patent holder of patents licensed to ZOLL Medical and Physio-Control. Olsen has received a research grant from ZOLL Medical to NAKOS. Hardig is employed by Physio-Control Inc/Jolife, the manufacturer and provider of the LUCAS device.

\section{Registration name and numbers:}

Paper I \& II: Clinical trial registration: NCT 00597207

URL: http://clinicaltrials.gov/show/NCT00597207

Paper III: Norwegian Animal Research Authority application ID (FOTS): 4931 


\section{Abbreviations and Glossary}

ACD-CPR - active compression-decompression CPR

AED - automated external defibrillator

AHA - American Heart Association

ALS - advanced life support

Asystole - lack of cardiac electrical activity

AutoPulse ${ }^{\circledR}$ - LDB based mechanical chest compression device

BLS - basic life support

$\mathrm{CCF}$ - chest compression fraction

CIRC - circulation improving resuscitation care

CODE-STAT ${ }^{\mathrm{TM}}$ - defibrillator data analysis software

$\mathrm{CPP}$ - coronary perfusion pressure

$\mathrm{CPR}$ - cardiopulmonary resuscitation

ECG - electrocardiogram

EMS - emergency medical services

ERC - European Resuscitation Council

$\mathrm{EtCO}_{2}$ - end tidal carbon dioxide

FDA - US Food and Drug Administration

ICD - implantable cardioverter defibrillator

ILCOR - International Liaison Committee on Resuscitation

$\mathrm{IQR}$ - inter quartile range

iA-CPR - integrated load distributing band (AutoPulse $($ ) CPR

LIFEPAK ${ }^{\circledR} 12 / 15$ - defibrillators 
LIFEPAK ${ }^{\circledR} 500$ - an AED

LDB - load distributing band

LDB-CPR - load-distributing band CPR

LUCAS ${ }^{\circledR}$ - piston based mechanical chest compression device

Manual CPR - CPR performed by human rescuer

NRC - Norwegian Resuscitation Council

OHCA - out-of-hospital cardiac arrest

PCI - percutaneous coronary intervention

PEA - pulseless electrical activity

$\mathrm{RCT}$ - randomized controlled trial

ROSC - return of spontaneous circulation

Shockable rhythms - VF \& VT

$\mathrm{SpO}_{2}$ - arterial oxygen saturation

SPSS - statistics analysis software by IBM inc.

TOF - termination of VF/VT

TTI - transthoracic impedance

VF - ventricular fibrillation

VT - ventricular tachycardia (pulseless) 


\section{List of papers}

\section{Paper I}

Steinberg MT, Olsen JA, Brunborg C, Persse D, Sterz F, Lozano M, Brouwer MA, Westfall M, Souders CM, van Grunsven PM, Travis DT, Lerner EB, Wik L. Minimizing pre-shock chest compression pauses in a cardiopulmonary resuscitation cycle by performing an earlier rhythm analysis. Resuscitation. 2015;87:33-7.

\section{Paper II}

Steinberg MT, Olsen JA, Brunborg C, Persse D, Sterz F, Lozano M, Westfall M, Travis DT, Lerner EB, Wik L. Defibrillation success during different phases of the mechanical chest compression cycle. Resuscitation. 2016;103:99-105.

\section{Paper III}

Steinberg MT, Olsen JA, Eriksen M, Neset A, Norseng PA, Kramer-Johansen J, Hardig BM, Wik L. Haemodynamic outcomes during piston-based mechanical CPR with or without active decompression in a porcine model of cardiac arrest. Scandinavian Journal of Trauma, Resuscitation and Emergency Medicine. 2018;26:31. 



\section{Introduction}

Ischaemic heart disease is the leading cause of death worldwide.(1, 2) Approximately $50 \%$ of all heart disease related deaths are due to sudden cardiac arrest.(3) It is estimated that emergency medical services (EMS) in Europe yearly attend to 86 out-of-hospital cardiac arrest (OHCA) victims per 100.000 person-years with great variation in reported incidence from 24 to 174 victims per 100.000 person-years.(4) In addition to possible variations in patient populations, this is partly due to factors such as different definitions of OHCA, which patients EMS starts to treat, and how data are collected and managed. Some of these definition problems were recognized almost three decades ago. In an attempt to better evaluate and compare studies of patients with OHCA, Utstein guidelines for uniform reporting of data were developed in 1991 as an international consensus process on definitions and concept that should be included in reports from and recommendations for emergency medical resuscitation systems.(5) After the publication of numerous studies it became apparent that the initial Utstein guidelines could benefit from revisions and expansions. Utstein guidelines were thereafter developed for in-hospital cardiac arrest,(6) paediatric cardiac arrest,(7) laboratory studies(8) and for post-resuscitation treatment factors,(9) with updates in 2004(10) and 2014.(11) The Utstein principles have also been used for trauma patients(12) and recently also for newborn and maternal survival in low-resourced countries.(13)

Overall survival to hospital discharge after OHCA has increased after decades of research and treatment improvements. $(14,15)$ But there are great variation between systems, and worldwide survival is reported between $0.6 \%$ and $31 \%$.(4) The Norwegian national cardiac arrest registry reported in 2016 that EMS treated 61 OHCA victims per 100.000 person-years, with a 30 day survival rate of $14 \%$, with $77 \%$ having a good neurologic outcome.(16) This gives a survival of 7.1 per 100000 inhabitants. In the same year, the Swedish Cardiopulmonary Resuscitation (CPR) registry reported a $11 \% 30$ day survival and the Danish registry $10.4 \%$, or 6.4 per 100000 inhabitants. $(17,18)$

There are several explanations for the variations in outcome, of which time factors and quality of the local chain of survival are probably the most important. Without early recognition, good quality early CPR, early defibrillation and post resuscitation care (figure 1.), survival is less likely.(19, 20) 


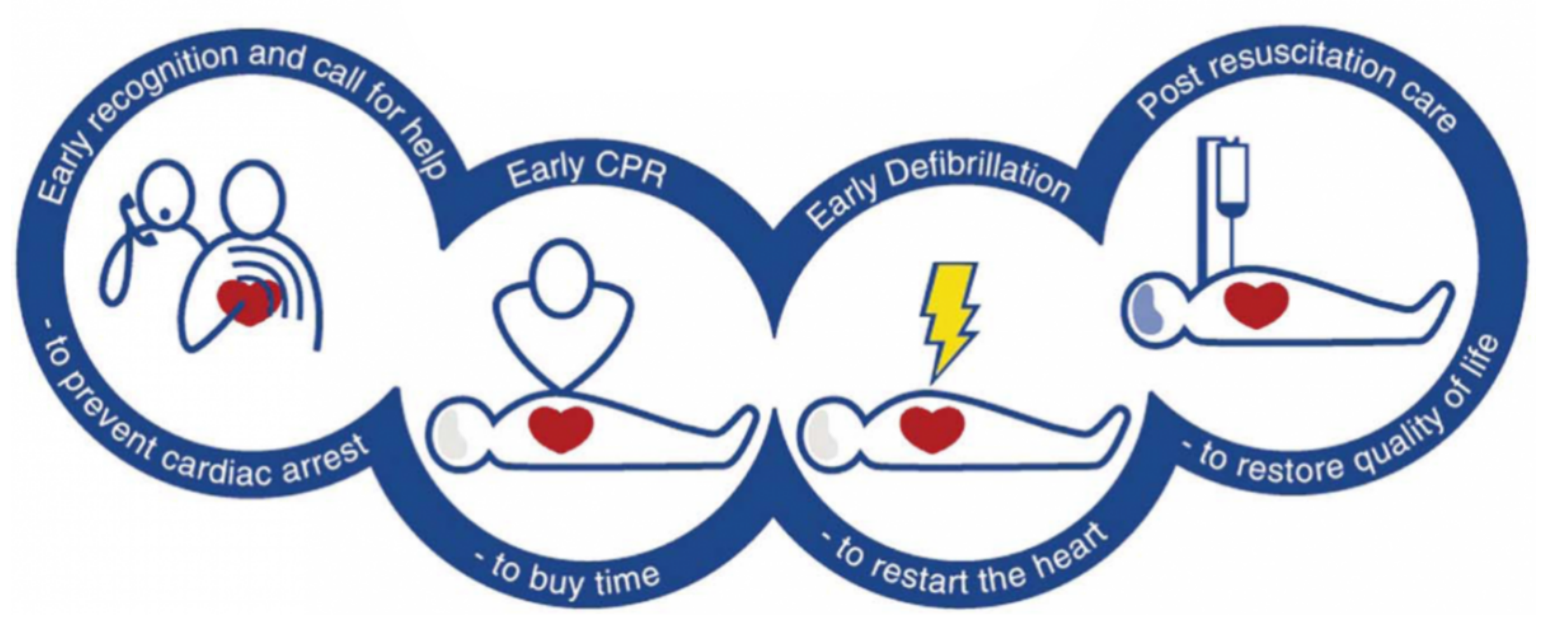

Figure 1. The chain of survival.(21)

By improving weak links in the local chain of survival, there was an improvement in survival in both Oslo(14) and Denmark(15). The fraction of patients with successful outcome can also partially be attributed to which patients EMS systems decide to treat, as well as differences in the fraction of patients with initial shockable cardiac rhythms (ventricular fibrillation (VF) and pulseless ventricular tachycardia (VT)), who have the highest probability of survival.(17) Non-cardiac cause of arrest and low rate and quality of bystander CPR reduce the proportion of initial VF/VT, thereby reducing the chance of a good outcome.(22-24) Untreated VF will deteriorate and turn into asystole, and cohorts have shown that survival would equalize if the percentage of patients with initial VF were the same.(4) There has been a worldwide reduction in patients with initial VF over time, which might partly explain why survival from cardiac arrest has not increased further in many countries.(25-28) It has been suggested that increased use of drugs such as $\beta$-blockers and treatment with percutaneous coronary intervention (PCI) in patients with coronary artery disease may have attributed to this decrease in patients presenting with VF. $(29,30)$ Others suggest that the increased use of implantable cardioverter defibrillators (ICD) in high-risk patients contribute to immediate cardioversion in patients having OHCA, thereby reducing the number of potential OHCA patients presenting with VF when personnel with an external defibrillator arrive.(31)

\section{The physiology and haemodynamics behind chest compressions}

Two main theories describe the physiology behind the blood flow generated by chest compressions. The heart pump theory, first described by Kouwenhoven et al in 1960, suggests that pressure applied to the sternum compresses the heart between sternum and spine, thereby forcing blood out of the heart, which due to functioning cardiac valves refills from the venous side when the pressure to sternum is removed.(32) The thoracic pump theory, first described 
by Rudikoff et al in 1980, suggests that chest compressions raise the intrathoracic pressure in general, thus closing off the low pressure great veins and squeezing blood antegrade out of the heart into the great arteries. Release of the chest compression thereafter generates negative intrathoracic pressure, and blood flows into the heart from the large veins.(33) Today, we assume that a combination of these two mechanisms is responsible for the circulation and vital perfusion created by CPR.(34)

The circulation generated by chest compressions delay ischaemic vital organ damage. Patient survival requires that the heart is restarted (return of spontaneous circulation, ROSC). In patients with initial asystole or pulseless electrical activity (PEA), the myocardial circulation generated by chest compressions in addition to ventilation, can be enough to restart the heart, depending on the cause of arrest, time factors and several other circumstances. $(35,36)$

\section{Bystander CPR}

Recent registry data from Sweden(15) and Denmark(23) have shown that bystander CPR not only increase survival 2-3 fold compared to no bystander CPR,(15) but also the number of neurologically intact survivors being able to work. $(23,37)$ Whether bystanders should do chest compression-only CPR or conventional CPR (including rescue breaths) has been debated for several years. Most studies have not been able to document differences in survival to hospital discharge(38-40) or survival with favourable neurological outcome between the two modalities.(41-44) The majority of these studies included adult patients with cardiac arrest of presumed cardiac origin. We might assume that patients who arrest due to hypoxic non-cardiac causes will benefit from rescue breaths in addition to chest compressions. Thus, in children where hypoxic non-cardiac causes of cardiac arrest are more common, one study found higher survival rates among patients who received conventional rather than chest compressions-only CPR.(45) Other studies indicate that there is a time threshold during CPR around 15 minutes, whereafter the absence of rescue breaths becomes harmful for the patient. $(46,47)$ European resuscitation council (ERC) and American heart association (AHA) conclude in their 2015 guidelines that untrained lay rescuers should perform chest compression-only $\mathrm{CPR}$ and that trained lay rescuers if able should give rescue breaths at a ratio of 30 compression to two ventilations $(30: 2)$. $(48,49)$ In a 2017 publication, the international liaison committee on resuscitation (ILCOR) states that continuous chest compressions with passive oxygenation is a reasonable alternative to 30:2 CPR for EMS providers in patients with witnessed arrest and a shockable rhythm.(50) It should be noted that the ERC, an ILCOR member organization, in a 2018 publication with some of the same authors as the ILCOR publication, recommends against this "minimally interrupted cardiac resuscitation" protocol as there is no evidence that this approach is superior to conventional 30:2 CPR.(51) 


\section{CPR quality}

The last decades of research and documentation have resulted in CPR guidelines emphasizing the importance of high quality CPR described as chest compressions with optimal rate, depth, chest wall recoil and as few interruptions as possible in addition to adequate ventilations.(52, 53)

Both ERC and AHA guidelines recommended in 2015 a chest compression rate of 100$120 / \min .(48,49)$ Both lower and higher rates reduce total coronary blood flow and are associated with decreased rates of $\operatorname{ROSC}(54-56)$ and survival.(54, 55) Increased chest compression depth is associated with increased ROSC(57-60) and survival(57, 60-62). ERC and AHA recommends today a chest compression depth of 4,5-6 $\mathrm{cm}$ (depending on the size of the patient).(48, 49) Too deep chest compressions might be associated with increased injuries and possible severe consequences.(63) In pigs with cardiac arrest, incomplete chest wall recoil reduced coronary perfusion pressure $(\mathrm{CPP})(58,64,65)$ and cardiac output.(58) This might be due to its impact on intrathoracic pressures reducing the filling of the heart. In anaesthetised children with spontaneous circulation, one study also found that leaning forces on the sternum reduced CPP, but without affecting cardiac output.(65) Even though we are lacking clinical data from cardiac arrest patients, both ERC and AHA recommend full chest recoil during chest compressions. $(48,49)$

\section{Advanced Life Support ventilation}

Advanced airway placement during resuscitation prevents pulmonary aspiration and makes it possible to ventilate during continuous chest compressions. In a recent randomised controlled trial (RCT) there was no difference in outcome between bag-mask ventilation and advanced airway management with endotracheal intubation.(66) It should be noted that the rate of initial asystole was high (approximately $72 \%$ ) and survival very low in both groups (approximately 4 $\%)$. In a large observational study survival rate to hospital discharge was higher with bag-mask ventilation than with an advanced airway (endotracheal tube or supraglottic device)(18\% vs $5.4 / 5.2 \%$, respectively).(67) In another large observational study survival to hospital discharge rates was similar for endotracheal intubation and bag-mask ventilation ( $8 \%$ vs $7 \%$ ), but lower for laryngeal masks (5.6 \%).(68) A recent large RCT did not show any differences in survival with favourable neurological outcome when comparing 30:2 CPR to continuous chest compressions (7.7 \% vs $7 \%$, respectively).(69) ERC and AHA make no recommendation regarding the use of bag-mask ventilation or advanced airway management during OHCA resuscitation. $(70,71)$. The use of ventilation techniques depends on local circumstances, skills 
and logistics, but if advanced airways are to be used, frequent training and quality control must be provided.

\section{Defibrillation}

Even though CPR alone can be enough to restart the heart under certain circumstances, ROSC is predominantly achieved after defibrillation of VF/VT. $(35,36)$ The purpose of defibrillation is to depolarize a critical mass of the heart muscle thereby terminating the myocardial fibrillation and create asystole. If the myocardium is in good enough metabolic condition, sinus rhythm may then be re-established by the sinoatrial node. Defibrillation is appropriate only when patients are presenting with VF or VT. Timing of defibrillation has been studied in order to identify a favourable approach to maximise termination of fibrillation (TOF), ROSC and survival. TOF is defined as termination of VF/VT $5 \mathrm{sec}$ after the shock has been delivered, and TOF rates are higher earlier in resuscitation attempts.(72) This is thought to be due to the dynamics of the metabolic state of the heart; a fibrillating heart consumes more oxygen than a beating heart.(73) Myocardial hypoxia occurs only a few seconds after occlusion of an coronary artery, and the energy production changes from aerobic to anaerobic pathways.(7476) Myocardial energy status, measured as adenosine triphosphate (ATP) levels in the myocardium and the ratio between ATP and adenosine diphosphate (ADP) decreases gradually during cardiac arrest.(77) Less time with VF/VT should therefore result in less myocardial oxygen and energy depletion with consequently higher likelihood of ROSC after defibrillation attempts.(78) Early and late resuscitation thus represent two different scenarios for defibrillation success. It has been demonstrated that TOF decreases significantly from the first to the fifth shock in patients with frequent refibrillations.(72) It has been suggested to divide resuscitation strategy into different phases based on time intervals from cardiac arrest and postulated that these phases demands different forms of therapy.(79) Consequently, defibrillation should be emphasized in both the electrical phase (up to approximately four minutes of cardiac arrest) and the circulatory phase (up to approximately 10 minutes of cardiac arrest), but might not be sufficient alone in the metabolic phase (after approximately 10 minutes of cardiac arrest).(79)

It was early discovered that the VF amplitude and frequencies depended on the duration of untreated VF with higher amplitude and frequency in patients treated shortly after cardiac arrest vs. low amplitudes and frequencies after longer arrest times.(80, 81) These studies also showed that outcome was better with significantly higher survival rates in patients with coarse VF. $(80,81)$ This led to further studies of computer models analysing VF waveforms mathematically during resuscitation in order to predict the chance of defibrillation success.(82) 
Strategies based on defibrillation success prediction algorithms have so far not increased the rates of ROSC or survival. $(83,84)$

Defibrillation using a biphasic waveform appears to increase the rate of TOF vs. a monophasic waveform,(85-91) and in one study survival rate increased.(92) This, in addition to the fact that it was accomplished with lower energy levels (120/150J for biphasic vs 200/300/360J for a monophasic waveform) and less damage to the myocardium,(93-97) is the reason why biphasic defibrillation technology has become standard the last decades.

Early recommendations for defibrillation advised up to three shocks in a row if the first one or two shocks failed. Studies have showed conflicting results regarding the outcome of a oneshock protocol versus three stacked shocks.(98-100) Focus on minimising pauses in chest compressions, and the idea that a failed shock might indicate the need for more myocardial circulation in order to enable TOF and ROSC, led to the recommendation of a single shock defibrillation strategy.(101-103) It has been reported that the majority of patients with VF refibrillate after successful TOF during resuscitation, $50 \%$ within two minutes after defibrillation. $(72,104)$ TOF success is highest for the first shock and declines significantly for the fifth shock, and this decline was less pronounced when a higher energy level (360J) was chosen for the subsequent shocks.(72) This is the rationale behind the ERC and AHA recommendation of an escalating energy protocol when technically possible. $(70,71)$

\section{CPR prior to defibrillation}

It has been hypothesised that coronary perfusion generated by CPR delivery before a defibrillation attempt can enhance the metabolic state of the heart, and thereby increase the likelihood of successful defibrillation.(105-110) In Seattle, they observed improvements in survival when 90 seconds of CPR before defibrillation was added to their local protocol.(111) In a RCT from Oslo, although showing no difference in overall outcome with $180 \mathrm{sec}$ of CPR prior to defibrillation vs standard care, subgroup analysis of patients with EMS response times above five minutes showed both increased rates of ROSC and survival with CPR before defibrillation.(112) Several RCTs have failed to find increased rates of ROSC(112-116) or survival(112-116) with delayed defibrillation in order to deliver a short period of CPR first, and three meta-analyses found no difference in outcome data.(117-119) An interesting posthoc analysis of an aforementioned RCT showed that when they looked at patients with VF and divided study-sites in those with good outcome vs bad (higher or lower than $20 \%$ survival), sites with poor outcome did worse with CPR first, but those with good outcome did better with CPR first.(120) It would appear that quality of CPR and advanced life support (ALS) have an 
impact. ERC and AHA do not recommend routinely delay of defibrillation in order to deliver CPR in their recent 2015 guidelines. $(48,49)$ The Norwegian Resuscitation Council (NRC) recommended 180 seconds of CPR before defibrillation in patients with cardiac arrest without bystander CPR untreated for more than five minutes in 2005 and 2010,(121) but changed into agreement with ERC and AHA in 2015, and is no longer recommending delaying defibrillation in any patients with initial VF. However, it is strongly recommended and emphasized that CPR should be performed until the defibrillator is attached to the patient and rhythm analysis being performed.(122)

\section{Cardiac rhythm analysis}

Before delivering a shock, chest compressions are normally discontinued. Several studies indicated that longer duration of this pre-shock period was negatively associated with shock success, $(123-125)$ ROSC $(126,127)$ and survival. $(128,129)$ One trial did not show any association between reduced pre- and peri-shock pauses and ROSC or survival.(99) Negative effects of chest compression pauses are also indicated by reports demonstrating that a lower fraction of time during resuscitation when chest compressions are given, called the chest compression fraction (CCF), is negatively associated with survival. $(130,131)$ A recent retrospective study reported that prolonged pauses in chest compressions in general were negatively associated with survival, not explained by CCF or decreased VF termination rate.(132) Based on these studies ERC and AHA suggest CCF should at least be above $60 \%$ with pauses in chest compressions no longer than $10 \mathrm{sec}$ when delivering rescue breaths or in association with defibrillation. $(48,49)$

During cardiac rhythm analysis, chest compressions need to be interrupted. This is because chest compressions result in artefacts on the ECG making cardiac rhythm analysis during chest compressions unreliable. There have been many attempts to filter out chest compression artefacts from the ECG to enable reliable analysis during chest compressions, but so far the sensitivity and specificity have not been sufficiently high.(133-137) One large retrospective study with a new algorithm discriminated recently between shockable and non-shockable cardiac rhythms during chest compressions with very high sensitivity and specificity.(138) No studies have looked at the effect of such filtering technologies on outcomes such as ROSC or survival in humans and ERC and AHA therefore advise against the use of such filtering technologies outside the scope of a research program. $(49,70)$ 


\section{Hands-on defibrillation}

Many measures have been taken to decrease pre-shock chest compression pauses. In the 2015 guidelines, $\mathrm{ERC}(52)$ recommends delivering chest compressions during defibrillator charging in order to minimize this chest compression pause.(130) Theoretically this pause can be reduced to almost zero, and the idea of defibrillating during continuous chest compressions has resulted in studies of so called "hands-on defibrillation". It has been shown that biphasic external defibrillation during mock-chest compressions from personnel with rubber gloves resulted in low levels of exposure to voltage leakage.(139) Another study concluded that an isolating defibrillation blanket was a safe and feasible way of delivering shocks during continuous chest compressions.(140) Concerns regarding the safety of these methods have been raised,(141) and one study concluded that standard nitrile gloves do not provide sufficient protection for hands-on defibrillation.(142) It has been emphasize in an editorial that despite the lack of documentation of serious injury to rescuers, a cautious approach should be used if one were to try to reduce or remove pre-shock pauses by hands-on defibrillation.(143)

\section{Mechanical chest compressions}

The first studies on mechanical CPR in the late 1970s and early 1980s utilised a pneumatically driven chest compressor(144, 145) mimicking chest compressions delivered by a human rescuer, and a pneumatic vest $(146,147)$ designed to emulate the circulation created by high intrathoracic pressures, inspired by the theory behind "cough CPR" and its ability to cause circulation during VF.(148) Both approaches demonstrated improved haemodynamic outcomes during CPR compared with manual chest compressions.(149, 150)

Many years have passed since these early devices were studied, and today several mechanical chest compression devices are commercially available, some of which are widely used in resuscitation both in- and out-of-hospital. The scientific evidence on the effect of these devices compared with manual chest compressions varies from none to large multi-centre RCTs. Neither Corpuls CPR ${ }^{\circledR}$ (Corpuls, GS Elektromedizinische Geräte GmbH, Germany) nor Lifeline ${ }^{\mathrm{TM}}$ ARM (Defibtech LLC, Guilford, CT, USA) devices have any published clinical evidence regarding their efficacy. Thumper ${ }^{\circledR}$ (Michigan Instruments, Michigan, USA) CPR has been compared to manual chest compressions in one retrospective study $(n=150)(151)$ and two RCTs ( $\mathrm{n}=150$ and $\mathrm{n}=20) .(152,153)$ The studies included both in-(151, 152) and OHCA patients,(153) and demonstrated improved $\mathrm{EtCO}_{2},(153) \operatorname{ROSC}(151,152)$ and survival rates.(152) 
Most clinical evidence is available for two mechanical chest compression devices; The Lund University Cardiac Arrest System; LUCAS ${ }^{\circledR}$ (Physio-Control/Jolife AB, Lund Sweden) and the AutoPulse ${ }^{\circledR}$ (ZOLL Medical, Chelmsford, MA, USA). LUCAS ${ }^{\circledR}$ is piston based (with a suction cup attached) and compresses the chest from above, similar to the early pneumatic chest compressor and chest compressions delivered by a human rescuer. It is thought to utilise both the heart- thoracic pump theories to generate blood flow. The AutoPulse ${ }^{\circledR}$ utilises a load distributing band (LDB) squeezing the chest, somewhat similar to the early CPR vest. It is also thought to generate some blood flow based on the heart pump theory, but probably mostly via the thoracic pump theory.(150)

These two devices have been in the centre of attention for a lot of research during the last decade. Both have demonstrated improved haemodynamic outcomes such as systolic blood pressure, $\mathrm{CPP}$, cardiac output, $\mathrm{EtCO}_{2}$, and cardiac and cerebral blood flow compared with manual chest compressions.(154-159) They have also been used to provide circulation during PCI and long episodes of resuscitation, i.e. cases of hypothermia and drowning.(160-163) Mechanical chest compression devices have an advantage over manual chest compressions when resuscitation is necessary during transportation and rescuer safety comes in conflict with high quality CPR.

While retrospective studies showed increased rates of ROSC and survival to hospital admission and discharge with AutoPulse ${ }^{\circledR},(164-166)$ the first clinical RCT with AutoPulse ${ }^{\circledR}$ was terminated after the first interim analysis because of inferior survival to hospital discharge rates compared with manual CPR.(167) A second RCT with AutoPulse ${ }^{\circledR}$, the circulation improving resuscitation care trial (CIRC), compared high quality manual CPR with mechanical CPR with integrated use of the LDB device with special attention to the implementation process. CIRC showed a small negative effect on ROSC for LDB-CPR, but equivalence regarding survival to hospital discharge.(168) No differences in injuries were found.(168) Another recent small study on the safety of mechanical CPR concluded that it could not be excluded that AutoPulse ${ }^{\circledR}$ CPR resulted in more serious injuries than manual CPR.(169) The same study concluded that LUCAS ${ }^{\circledR}$ CPR did not result in more serious injuries. 


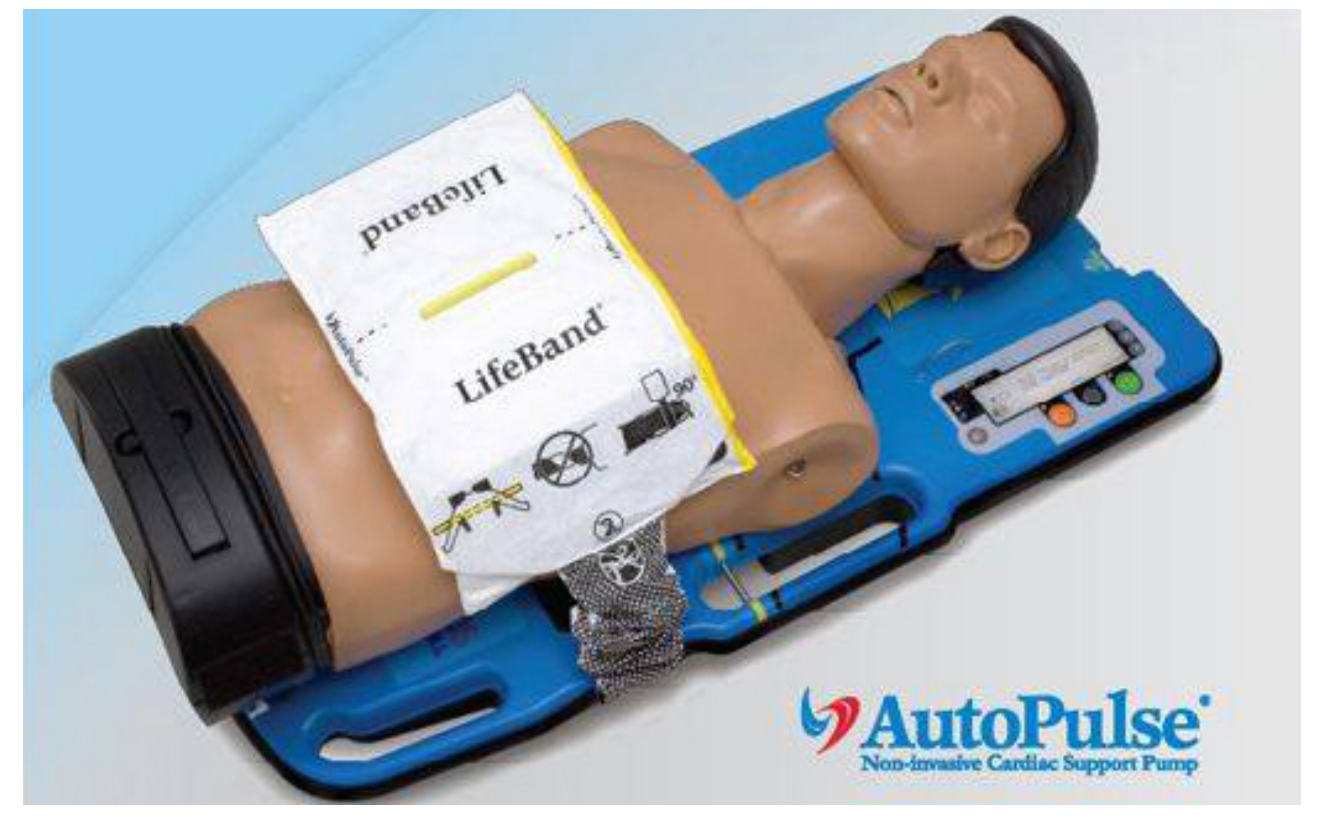

Figure 2. AutoPulse ${ }^{\circledR}$ Load distributing band chest compression device.

LUCAS $^{\circledR}$ has been studied in two small pilot $\operatorname{RCTs}(170,171)$ and in two large multi-centre RCTs, $(172,173)$ none of which showed any differences between manual and mechanical CPR regarding ROSC or survival. In their 2015 guidelines, ERC advise against routinely use of mechanical chest compression devices instead of manual chest compressions, but they emphasise that they can be used in special circumstances.(70) AHA 2015 guidelines suggests that mechanical CPR with a piston-based or LDB device may be a reasonable alternative for poorly trained personnel and in special situations when it is difficult to deliver consistent high quality manual CPR.(53) Despite the lack of RCT documented improved survival with mechanical chest compression, $45 \%$ of EMS services participating in a US cardiac arrest registry reported that they used mechanical CPR devices in 2012.(174) 


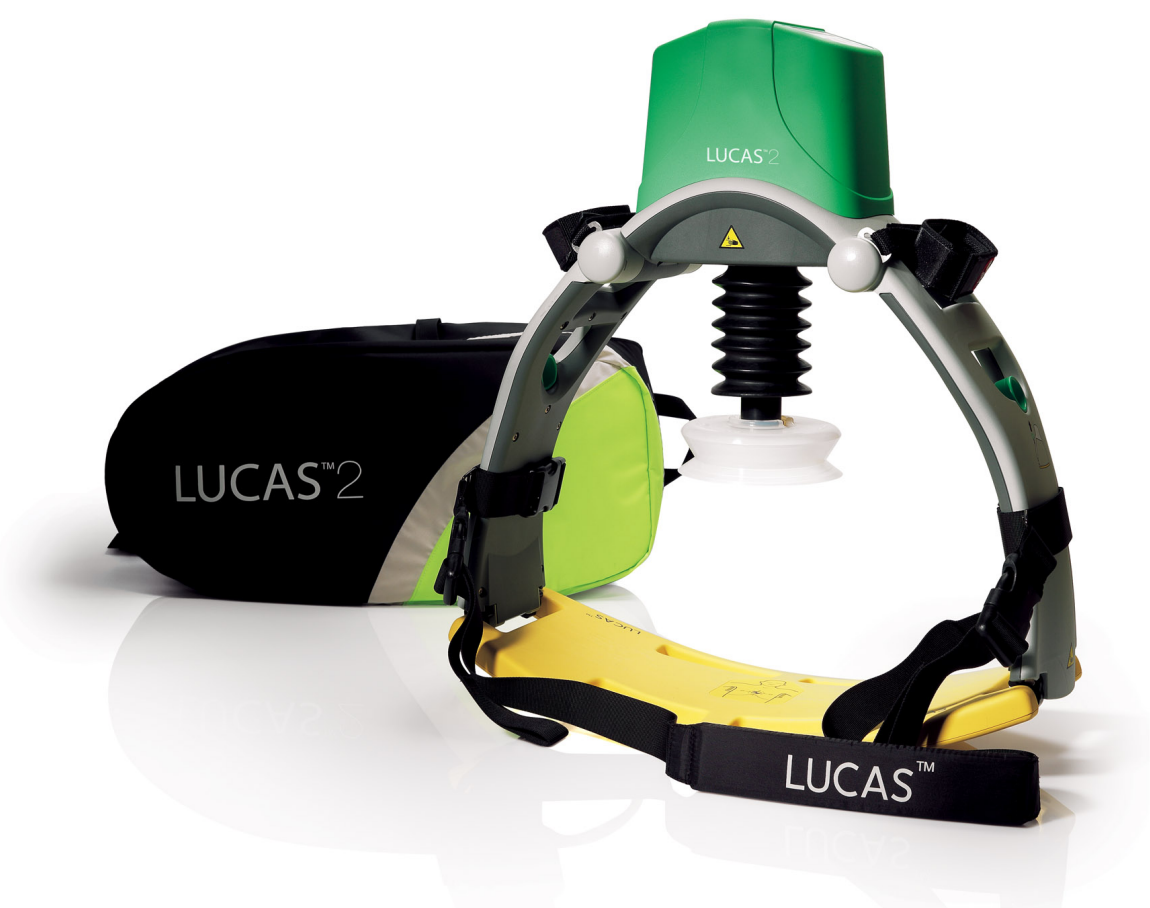

Figure 3. LUCAS $2^{\circledR}$ (Physio-Control/Jolife) piston-based chest compression device.

Mechanical chest compression devices represent both a way to deliver consistent CPR according to guidelines and enable delivery of shocks during CPR, without posing a risk to the rescuers. The protocols of the recent three large RCTs comparing mechanical to manual chest compressions all called for patients who received mechanical chest compressions to be defibrillated during continuous chest compressions.(168, 172, 173) Even though the protocol warranted shocks to be delivered during chest compressions, many patients in the CIRC trial $(52 \%)$ were defibrillated after a halt in chest compression. This was studied in a secondary analysis where TOF success rate was compared between shocks delivered in a chest compression pause and shocks delivered during chest compressions (zero chest compression pause).(175) This study identified that shocks with zero chest compression pause achieved lower TOF compared with those delivered after a chest compression pause of various lengths (1-9 sec: $26 \%$, 10-19 sec: $15 \%, 20-29$ sec: $7 \%$ and above 30 sec: $5 \%$, Chi squared, first shock: $77 \%$ TOF, $\mathrm{p}=0.05)$.(175) These results go against most studies regarding pre-shock pauses $(123,125,126,128,129)$ and might indicate that defibrillation during chest compressions may be suboptimal. Two studies have defibrillated pigs in different phases of the chest compressions cycle and found that shocks delivered in the upstroke (decompression) phase of both mechanical and manual chest compressions resulted in increased TOF rate 
compared to shocks delivered after a two-seconds chest compression pause. $(176,177)$ No clinical trial has to my knowledge studied the timing of defibrillation during mechanical chest compressions.

\section{Active compression-decompression CPR}

The concept of manual active compression-decompression CPR (ACD-CPR) with the help of a toilet plunger was first described in a successful resuscitation case by Lurie et al in 1990.(178) They suggested that ACD-CPR generated a negative intrathoracic pressure during active decompression, which also generate ventilations.(178) The idea was studied in both dogs and humans with a handheld plunger device, which increased systolic blood pressure, CPP, cardiac output and $\mathrm{EtCO}_{2}$ compared to both mechanical and manual CPR.(179, 180) Further studies indicated that active decompression indeed generated negative intrathoracic pressure increasing venous return and ventricular filling, thereby improving both blood pressures and cardiac output.(179, 180) ACD-CPR was also reported to improve myocardial and cerebral blood flow(181) and minute ventilation.(182) Further experimental studies on ACD-CPR continued to demonstrate improved haemodynamic outcomes in animals.(183-185)

Clinical studies on ACD-CPR emerged at the same time as the haemodynamic benefits were discovered in the early 1990s. A series of haemodynamic studies on the ACD-CPR effects on humans found results similar to the animal data with increased arterial pressure, $\mathrm{CPP}, \mathrm{EtCO}_{2}$ and minute ventilations, $(179,186-189)$ but two studies failed to demonstrate haemodynamic benefits of ACD-CPR in humans.(190,191) These results were followed by a series of clinical trials, all but one(192) failed to demonstrate any short or long term survival differences between ACD-CPR and standard manual CPR.(193-200)

The combination of ACD-CPR and an impedance threshold valve (ITV) has also been studied. An ITV is thought to augment negative intrathoracic pressure when combined with ACD-CPR by impeding inspired air during the decompression phase of chest compressions.(201) One study reported improved rates of ROSC and short term survival with this combination(202) and a second RCT and a secondary analysis of this trial indicated increased survival to hospital discharge with favourable neurological outcome. $(203,204)$ These two publications from the same study have been criticised for high sponsor involvement $(203,204)$ and were categorized by ILCOR as very low quality with serious risk of bias, inconsistency and imprecision.(101) ILCOR have failed to reach a consensus regarding the combined use of ACD-CPR and an ITV,(101) while ERC recommend against routine use of this combination because of the high number needed to treat.(70) 
All studies on ACD-CPR, up until now, have utilised a handheld device (CardioPump ${ }^{\circledR}$ ) or a large power driven customised mechanical chest compression device. These mechanical devices were impossible to bring into the field and use in the clinical setting of cardiac arrest. The handheld ACD-CPR device has been reported to require more energy than regular CPR, and CPR quality has been documented to suffer.(205-207) A study on exertion during ACDCPR and standard CPR conclude that ACD-CPR requires $25 \%$ more work than regular CPR, which could affect CPR performance especially during prolonged resuscitation attempts.(207) A manikin study on CPR quality comparing manual ACD-CPR and regular CPR demonstrated that the ACD-CPR group delivered chest compressions with significantly lower depth, rate and chest compression fraction compared to conventional CPR. No participant was able to follow ERC CPR guidelines while using the handheld ACD-CPR device, and only $18 \%$ managed to deliver the manufacturer's recommended decompressions force.(206) Similar results were found in a second manikin study comparing both mechanical CPR and conventional CPR to ACD-CPR. ACD-CPR was associated with both lower chest compression depth, rate and fraction compared with manual CPR, and decompression force was inadequate $46 \%$ of the time.(205) Whereas the handheld ACD-CPR results seem to be far away from fulfilling todays ERC recommendations on CPR quality, mechanical ACD-CPR would probably be able to deliver high quality CPR according to today's ERC guidelines. A portable mechanical chest compression device able to deliver consistent high quality ACD-CPR, and thereby used in clinical trials of OHCA would bring additional scientific information to the efficacy of ACDCPR.

\section{Electronic defibrillator data}

Today's defibrillators can monitor and store a great number of different data from the resuscitation episode. This has enabled evaluation of CPR performance both during and after resuscitation and has provided OHCA researchers with large amounts of accurate data. In addition to recording patient data, blood pressure, timing of shocks, and shock energy, many of today's defibrillators can monitor and store continuous data curves from temperature, $\mathrm{SpO}_{2}$, $\mathrm{EtCO}_{2}$, 3-lead ECG, 12-lead ECG, pads-ECG, transthoracic impedance (TTI) and accelerometer measurements. The defibrillator pads record ECG and TTI when no other monitoring equipment is connected to the patient. Electrical impedance is defined as the total opposition that a circuit presents to an electrical current.(208) TTI in a patient's chest is proportional to the voltage generated by a high frequency, low amplitude alternating current sent between the defibrillator pads. Defibrillators can this way present a continuous TTI signal throughout the resuscitation attempt as long as the defibrillator pads are placed. TTI data have 
been used for decades in cardiopulmonary research,(209-212) and can be used to monitor both ventilations(213) and chest compressions(214) during resuscitation. Blood and bodily fluids are good electrical conductors, and therefore TTI is reduced when aorta fills. Air is a poor electrical conductor, and TTI will rise when the lungs fill with air.(214) These data can then be transferred to a computer and analysed using appropriate software. If there are no artefacts or impedance changes, the TTI curve will be a flat line.

a.

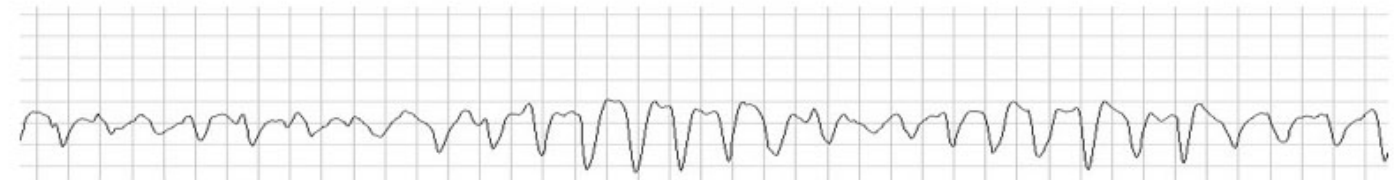

b.

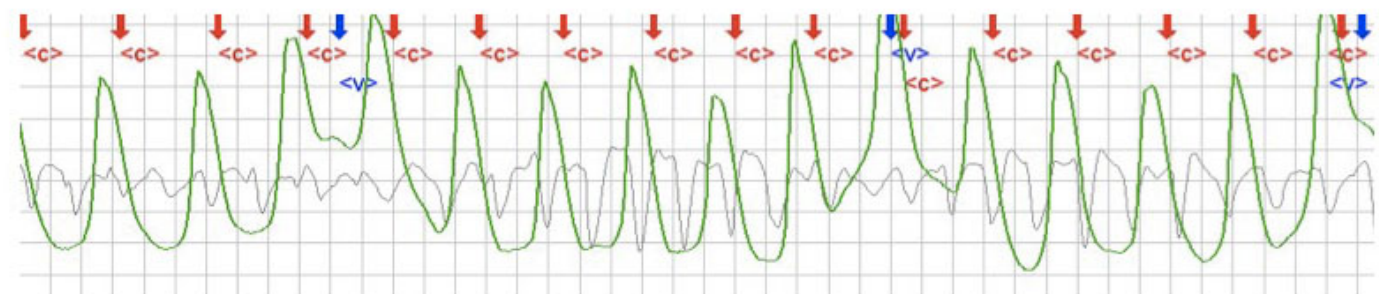

C.

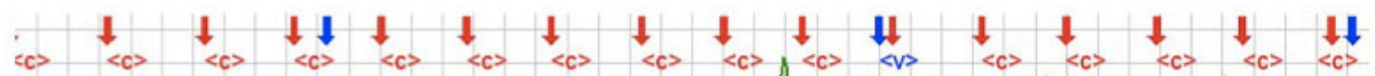
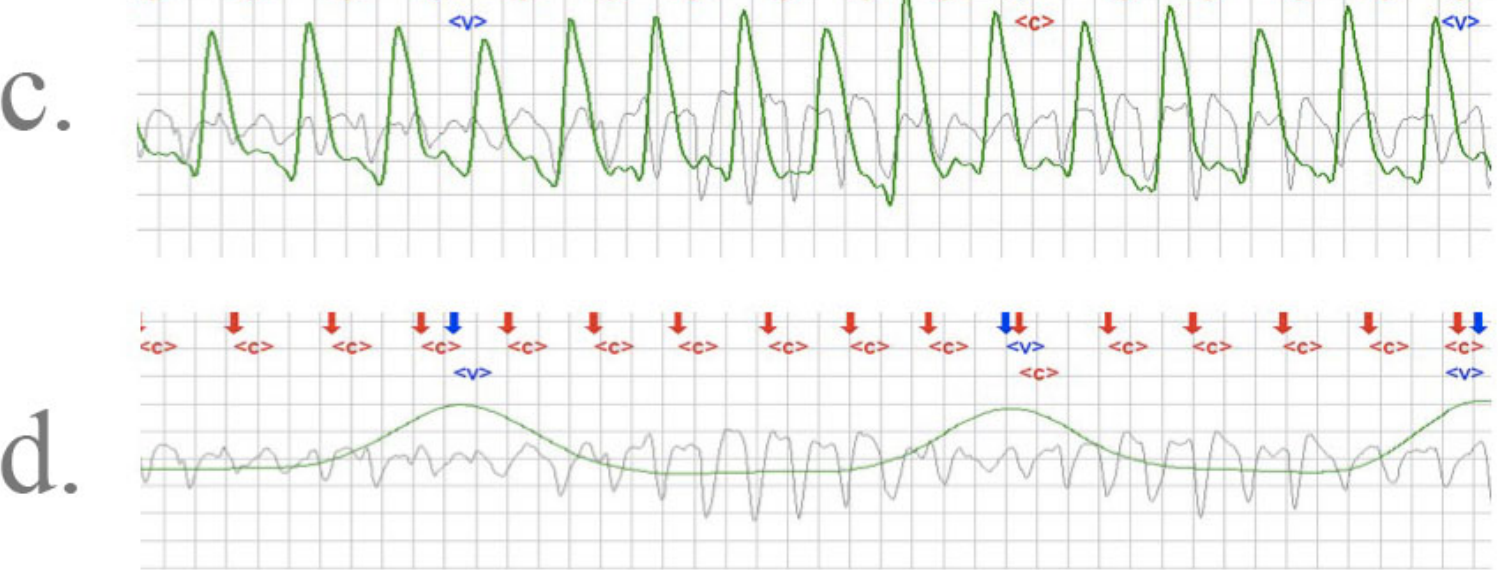

Figure 4. Screenshot from CODE-STAT ${ }^{\mathrm{TM}}$ (Physio-Control) displaying:

a. ECG rhythm (grey).

b. Unfiltered TTI signal showing both chest compressions and ventilations (green).

c. Filtered TTI signal showing only chest compressions.

d. Filtered TTI signal showing only ventilation. 


\section{Aims of the thesis}

The overall aim of the thesis is to study details of the relationship between CPR and defibrillation during resuscitation of cardiac arrest. Due to the design of the present studies, with two retrospective secondary analyses of the randomized multicentre CIRC trial, and one animal experimental model, they are meant to be hypotheses generating only.

Aims of the three papers:

Paper I - To retrospectively study whether or not a shockable rhythm detected one minute after a shock is still shockable three minutes after the shock, with two minutes of either mechanical or manual CPR in-between. Further, to evaluate the possibility of removing the pre-shock pause for rhythm evaluation based on the predictive value of an earlier rhythm analysis.

Paper II - To retrospectively study defibrillation success measured as TOF during continuous mechanical chest compressions in relation to where in the chest compression cycle the shock is delivered.

Paper III - To study if a mechanical chest compression device modified to deliver ACD-CPR improves haemodynamic outcomes compared to regular mechanical chest compressions in an experimental pig model. 


\section{Material and Methods}

\section{The Circulation Improving Resuscitation Care trial - CIRC}

CIRC was a multi-centre RCT consisting of emergency medical services (EMS) and hospitals in Europe and the US. It was carried out in Houston, TX, Fox Valley, WI and Hillsborough County, FL in the US, Vienna in Austria and Nijmegen in the Netherlands. Eligible patients were above 18 years old and suffered from OHCA of presumed cardiac aetiology. Participating sites represented different emergency response systems with both ALS and basic life support (BLS) delivered from fire and rescue services, and rescue services with physicians or nurses.(215)

CIRC was carried out under exception from informed consent for emergency research as outlined by FDA regulations in the US and similar laws of the Netherlands and Austria. The trial was approved by institutional review boards at US sites and ethics committees at European sites, and at participating EMS and hospitals likely to receive patients.(168, 215)

The aim of CIRC was to compare integrated mechanical LDB-CPR (iA-CPR) to high quality manual CPR (M-CPR) with survival to hospital discharge as main outcome. Secondary outcomes were ROSC, 24h survival and neurological status at hospital discharge.(215) When resuscitation was indicated, all patients received M-CPR initially and were randomized on site (1:1), by opening an envelope, to either continue with M-CPR or change to the LDB device (integrated AutoPulse ${ }^{\circledR}$ (iA)-CPR). American and European study sites followed AHA and ERC guidelines, respectively, with the exception that CPR cycles were three minutes and rhythm analysis including palpating a pulse was carried out one minute after defibrillation as in the Norwegian 2005 ALS Guidelines.(121, 215) The trial included several features in order to optimize protocol compliance, EMS deployment training, pre-trial studies of provider compliance, and three distinct trial phases, in-field training, run-in and statistical inclusion.(215) 


\section{ADULT ADVANCED CARDIAC LIFE SUPPORT}

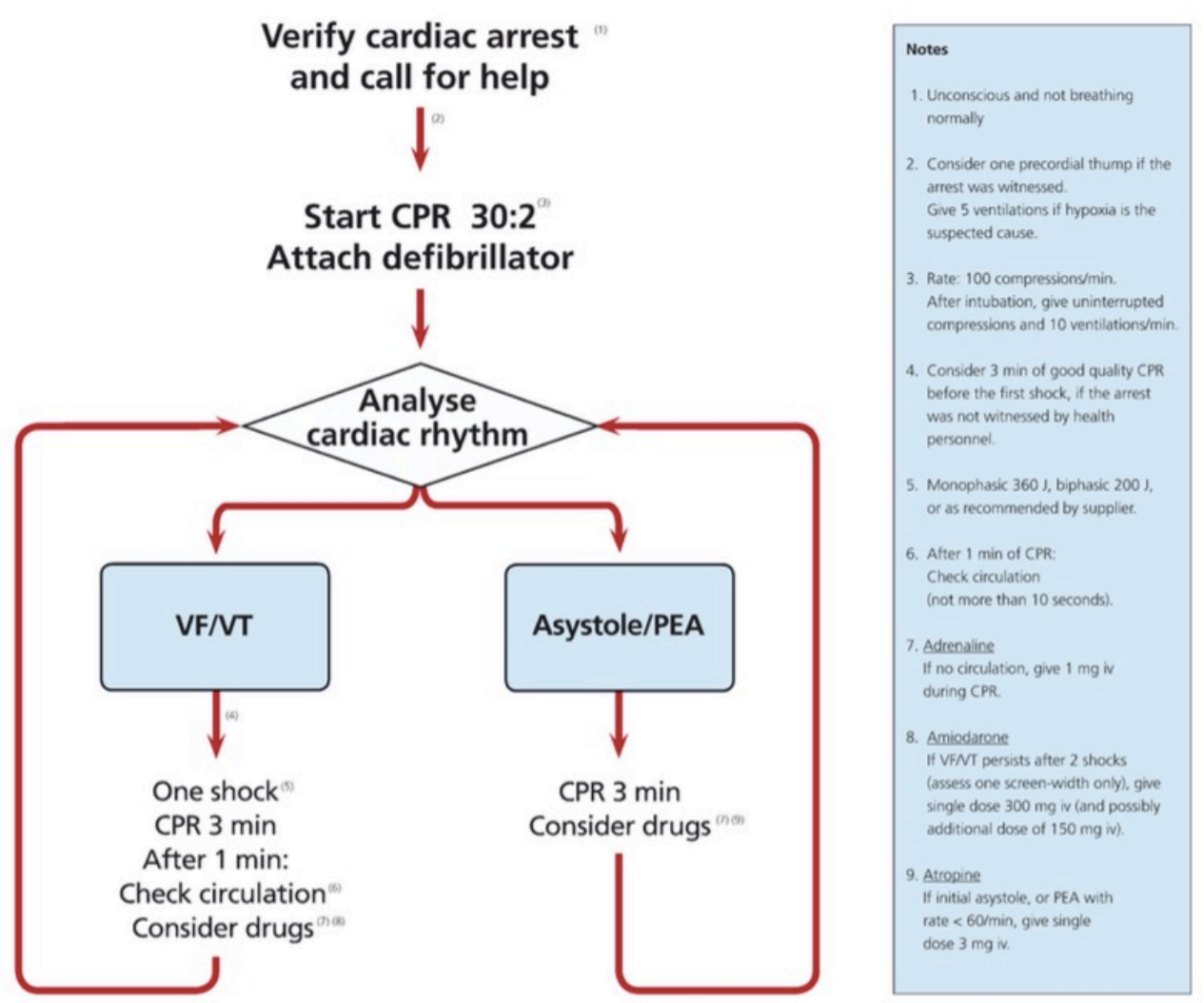

Figure 5. Norwegian resuscitation council 2005 advanced life support algorithm.(121)

A total of 4753 patients were randomized between $5^{\text {th }}$ of March 2009 and $11^{\text {th }}$ of January 2011 , and 4231 patients were included for analysis. The M-CPR group and the iA-CPR group were similar regarding all factors but initial shockable cardiac rhythm, which was higher in M-CPR (M-CPR $24 \%$ iA-CPR $21 \%$, OR 1.18, $95 \%$ CI 1.02-1.36, p=0.02).(168) Intention to treat analysis regarding main outcome survival to hospital discharge, adjusted for covariates, demonstrated equivalence (predefined to OR 0.69-1.44) between M-CPR and iA-CPR (M-CPR $11 \%$, iA-CPR $9.4 \%$, OR 0.89, $95 \%$ CI 0.72-1.10).(168)

\section{Paper I}

\section{Study setting \& population}

Paper I is a retrospective study on whether or not a shockable rhythm detected one minute after shock continues to be shockable after two minutes of either mechanical or manual CPR. It is based on continuous electronic defibrillator data collected and stored from the CIRC trial. Data from $96 \%$ of all patients were available for analysis.(168) Data included ECG from defibrillator pads, TTI, number and timing of shocks in addition to $\mathrm{EtCO}_{2}$ and 12-lead ECG when available. Defibrillators used were $\operatorname{LIFEPAK}^{\circledR} 12,15$ and 500 (Physio-Control, 
Redmond, WA, USA) and Zoll E series ${ }^{\circledR}$ and AED Pro ${ }^{\circledR}$ (Zoll Medical, Chelmsford, MA, USA).(168) All participating sites used anterior-lateral pads positioning. The energy protocol used was either fixed at 360 Joules or escalating from 200 - 360 Joules.

\section{Data review \& Study design}

Electronic data files were reviewed using CODE-STAT ${ }^{\text {TM }} 8.0$ or 9.0 (Physio-Control, Redmond, WA, USA) or RescueNet ${ }^{\circledR}$ Code Review 5.5.3 (Zoll Medical, Chelmsford, MA, USA). Chest compressions and ventilations were annotated using TTI.(213, 214) Manual and mechanical chest compressions were distinguished based on the constant rate and distinct morphology of the TTI graph produced by the LDB device. Cardiac rhythm was evaluated by ECG and the following rhythms were annotated by both MS, JAO and LW independently: Initial rhythm, pre-shock, five sec post-shock, one minute post-shock and three minutes postshock.(175) Rhythm annotations were mainly carried out during periods without chest compressions, except in episodes when the patient received continuous chest compressions without pauses for ventilation. In these episodes, the rhythm was annotated through chest compressions, and the first pause in chest compressions was used to confirm the annotation. If these rhythms were different from each other, the rhythm was marked as unknown if no consensus was reached.(175) ROSC was annotated based on ECG, TTI, ETCO 2 and notes in the ambulance records. Shocks delivered in periods with asystole, PEA or ROSC were categorized as inappropriate. To evaluate the predictive value of the one minute rhythm analysis, all shocks were included regardless of initial rhythm. Shocks with missing TTI or ECG, or with ICD shocks between the one and three-minute rhythm analyses were excluded. Due to the importance of an artefact free ECG in this evaluation, shocks without a minimum two sec pause in chest compressions for rhythm analysis were also excluded.

\section{Statistical analyses}

Descriptive statistical analyses were performed using SPSS version 21.0 (IBM SPSS Inc., Chicago, IL, USA) when studying the predictive value of cardiac rhythm evaluation one minute after the shock. Time intervals are presented as medians with inter quartile ranges (IQR) and $25 \%$ and $75 \%$ percentiles. Only descriptive analyses were performed, and multiple imputations were not used since defibrillation is the dependent variable with repeated measurements, thereby not contributing with information to the analyses.(216) 


\section{Paper II}

\section{Study setting \& population}

Paper II is a retrospective study on continuous electronic defibrillator data collected and stored from the CIRC trial. The study compares defibrillation success, when shocks are delivered during continuous chest compressions, with regards to when in the compressiondecompression cycle shocks are delivered. The data available for analysis were the same as in paper I and data characteristics are mentioned in the similar chapter for paper I.

\section{Data review \& Study design}

Electronic data files were analysed using the same software as in paper I. The methods regarding cardiac rhythm annotations and analyses were also identical to paper I. When evaluating the effect of defibrillation during different phases of the chest compression cycle, the up-to-three first three shocks from patients with initial shockable rhythm and LDB-CPR prior to defibrillation were included for analysis. Shocks were then categorized as shocks during chest compressions or shocks during a chest compression pause, based on TTI.(214) The latter group were defined as the control group, consisting of shocks delivered after LDBCPR, but during a chest compression pause. The former group, consisting of shocks delivered during chest compressions, was further categorized into the following phases of the LDB chest compression cycle: Compression phase (LDB tightens and stays tight), decompression phase (the LDB loosens) and the relaxation phase (the LDB stay loose between compressions), see figure 6 . 


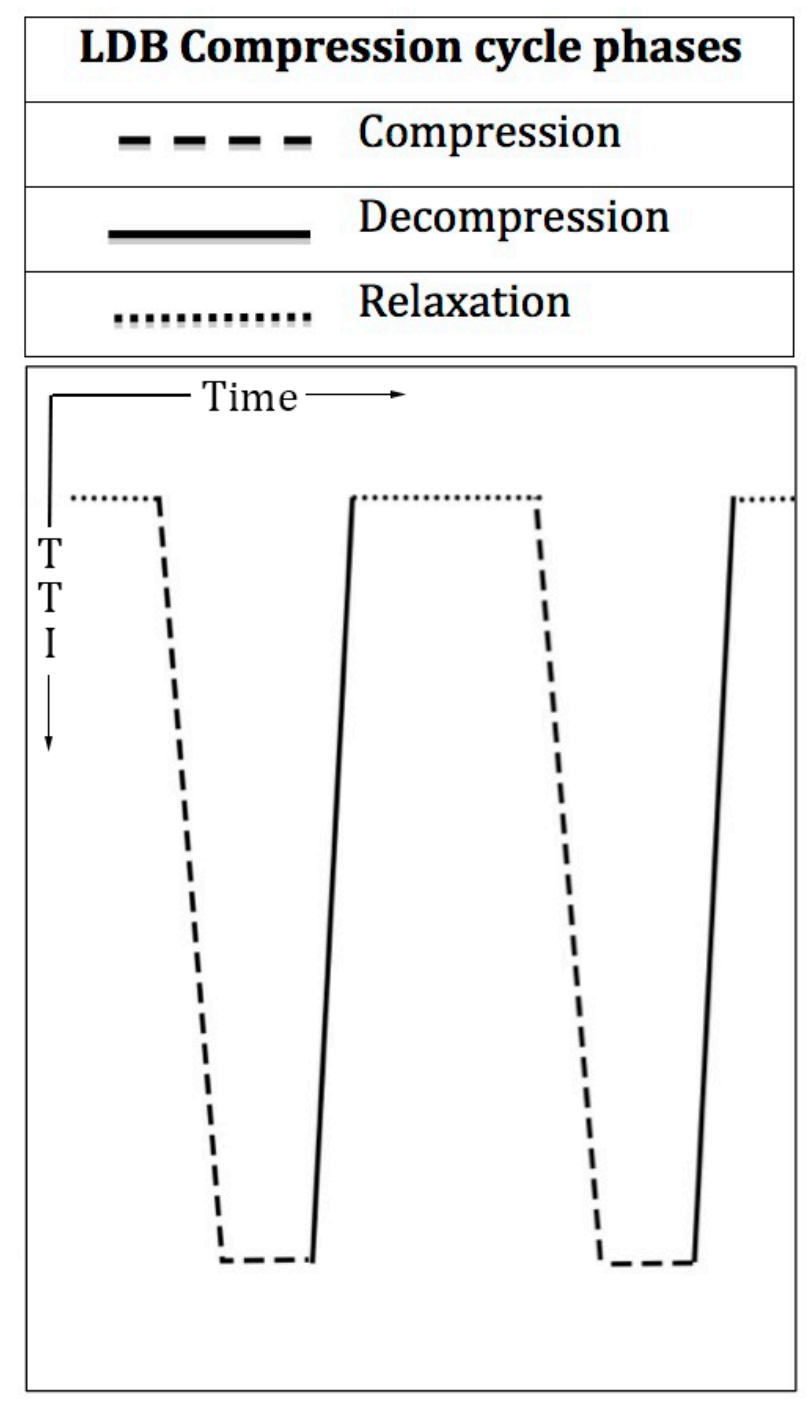

Figure 6. LDB Compression cycle phases

This categorization was based on the morphology and constant rate of chest compressions delivered by the LDB device, and knowledge of the LDB duty cycle provided by Zoll Medical. The determination of each shock to the specific chest compression cycle phase was carried out by using a transparent with a printed grid of the LDB duty cycle scaled to $50 \mathrm{~mm} / \mathrm{sec}$, the same calibration and scaling were applied to the electronic ECG and TTI data in the software CODE-STATTM . Aligning the LDB duty cycle on the transparent with the TTI graph in CODE-STAT $^{\mathrm{TM}}$ (see figure 7.) allowed for determination of in what phase of the chest compression cycle the shock was delivered with a margin of error of $25 \mathrm{msec}$ for shocks from LIFEPAK $^{\circledR} 12 / 15$ defibrillators and 35 msec for LIFEPAK ${ }^{\circledR} 500$ defibrillators. 

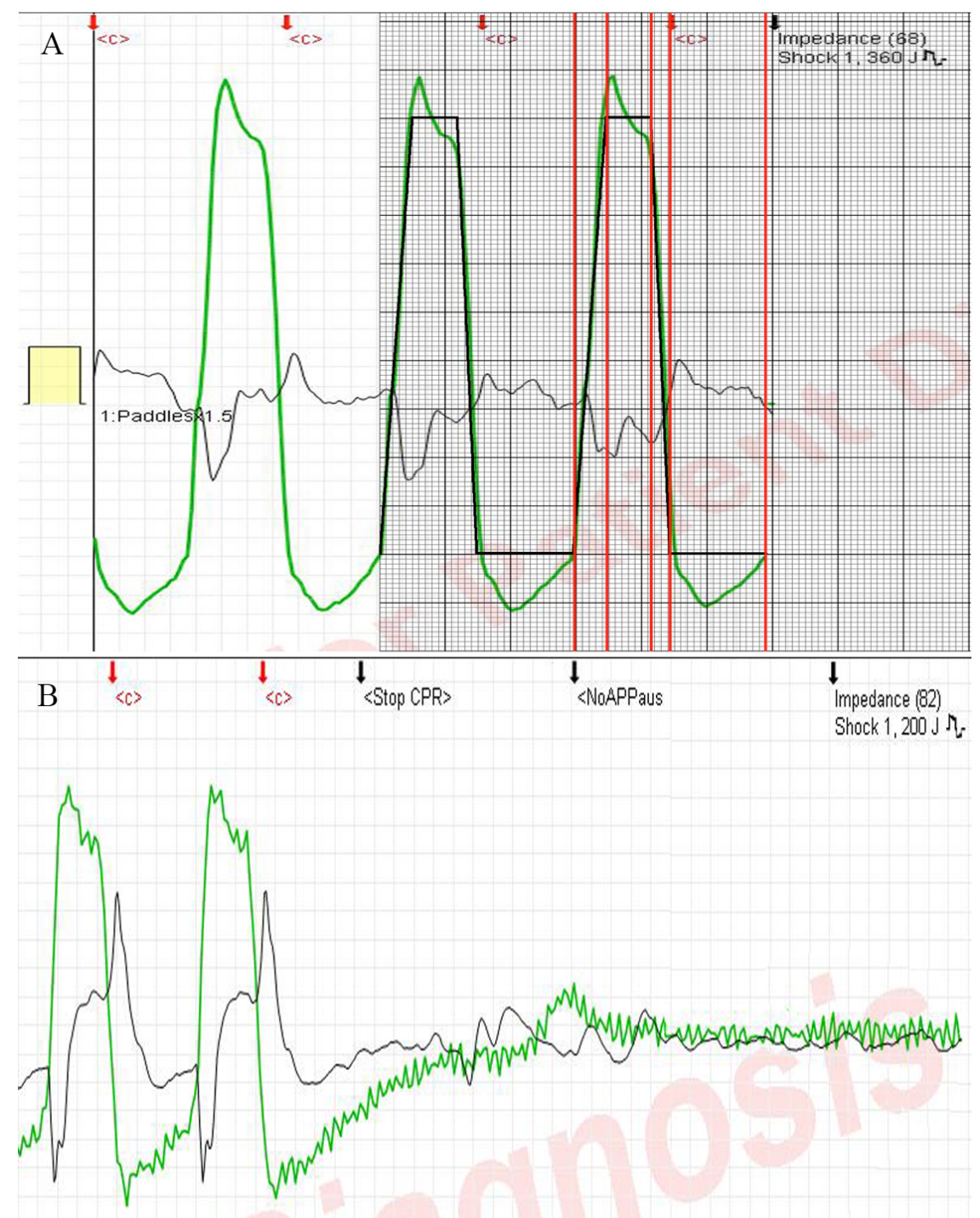

Figure 7. Screenshots from CODE-STAT TM displaying a TTI graph (green) and ECG strip (grey). A: Shows a shock delivered during chest compressions with the overlaying transparent grid on the right side displaying the LDB duty cycle. The black arrow indicates when the shock is delivered. B: Displays a control shock delivered after a pause in chest compressions. 


\section{Statistical analyses}

When analysing defibrillation success during different phases of the chest compression cycle, two analyses were performed; one included only the first shock from each patient and the second included the up-to-three first shocks per patient. Statistical analyses were performed using SPSS (version 22.0) and Stata ${ }^{\circledR} 14$ (StataCorp LP, College Station, TX, USA).

Defibrillation success was the main outcome defined as termination of fibrillation (TOF) five seconds post-shock (removal of VF or pulseless VT). Patients were not randomized to receive shocks in specific chest compression cycle phases, and one patient could therefore contribute with shocks in several different phases. The first shock group contained independent patient observations and logistic regression was used to compare TOF rates. Results were presented unadjusted and adjusted for witnessed arrest, bystander CPR, defibrillator shock energy and impedance. The up-to-three shocks group did not contain independent patient observations and to account for repeated measures and possible correlations the general estimating equations (GEE) model with exchangeable matrix structure was used for TOF rate comparisons in the up-to-three shocks analysis. Adjustments were made for defibrillator shock energy and impedance, since these factors were identified as possible patient independent confounding variables for defibrillation success. P-values $<0.05$ were considered significant. Inter-rater agreement was analysed for both post-shock rhythm annotations and for determination of shocks to different chest compressions cycle phases and calculated by unweighted Kappa statistics on random samples selected by SPSS (version 22.0). Kappa values $>0.81$ were considered excellent agreement.

\section{Paper III}

\section{Study setting \& design}

In this prospective experimental animal porcine cardiac arrest model, regular mechanical chest compressions were compared to ACD-CPR delivered by a modified LUCAS 2 device. The study was designed as a randomized controlled trial were each pig served as its own control in cross-over design. Healthy Norwegian domestic pigs of both genders were studied in the laboratory at the Institute of Experimental Medical Research, Oslo University Hospital. The study was carried out in accordance with "Regulations on animal experimentation" under the Norwegian Animal Welfare Act and approved by Norwegian Animal Research Authority. The ARRIVE Guidelines Checklist for animal research were enclosed in the publication process. 
The pigs were anaesthetised initially with ketamine (intramuscular), followed by a continuous infusion of propofol and fentanyl intravenously and put on a ventilator while preparation and instrumentation of all catheters and monitors were placed. This included bladder temperature, doppler flow meter on the carotid artery, pressure transducer catheter in the aortic arch and right atrium, catheter in the pulmonary artery for thermodilution cardiac output and wedge pressure measurements, catheters in aorta and right atrium for blood gases, pressure transducer in oesophagus for intrathoracic pressure and craniotomy with placement of laser doppler flowmeter on the surface of cerebral cortex. A metal plate was screwed on to the pig's sternum in order to connect the piston from the LUCAS 2 device used in the experiment to the pig's chest.

A LUCAS 2 device was modified (no suction cup, software re-programming) to deliver both standard mechanical CPR and active decompression two $\mathrm{cm}$ above resting chest level. The combination of five $\mathrm{cm}$ of compression and two $\mathrm{cm}$ of decompression was chosen based on an earlier porcine study comparing different degrees of both compressions and active decompressions during ACD-CPR.(185) VF was induced by a transcardial DC current and left untreated for two minutes. Three 180-second sequences with CPR were performed on each pig, which were randomized to either standard CPR, ACD-CPR, standard CPR or ACD-CPR, standard CPR, ACD-CPR. Changes between different techniques were carried out by placing or removing a metal pin, which fastened the LUCAS piston to the sternal plate. Pressure and flow variables were recorded continuously, and cardiac output and blood gases measured twice and once respectively per CPR sequence.

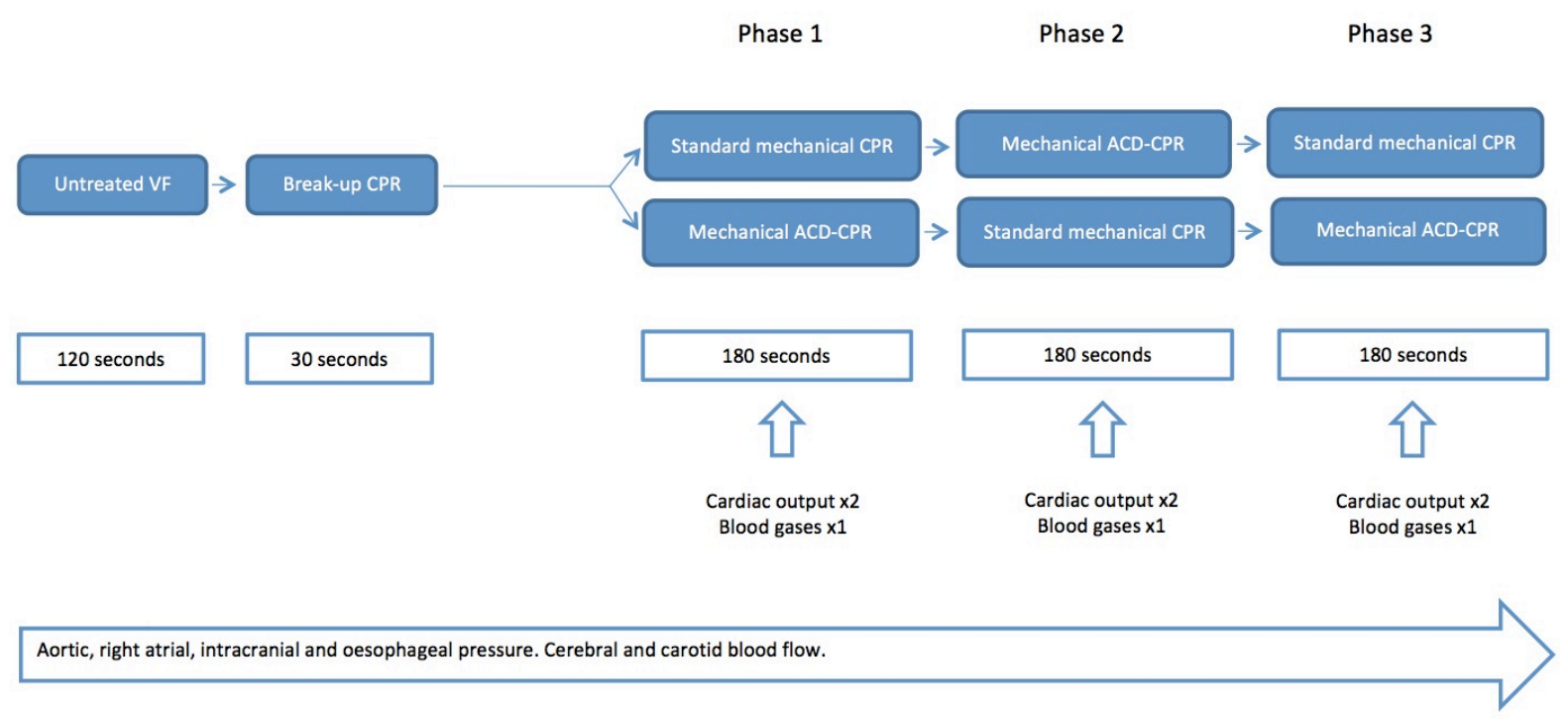

Figure 8. Illustration of the experimental protocol. 
Continuously measured pressure and flow signals were sampled with a PC data acquisition system (NI SCXI-1000, NI PCI-6036E, National Instruments Company, Austin, TX, USA) with VI Logger software (National Instruments Company, Austin, TX, USA) and broken down to a sampling frequency of 100 per chest compression cycle.

\section{Statistical analyses}

For the primary outcome of CPP, in order to demonstrate a $10 \mathrm{mmHg}$ difference with the power of 0.99 and alpha 0.05, power analysis (Sample Power, HyLown Consulting LLC, Atlanta, GA, USA) showed that 10 pigs were required using paired analysis in a crossover design. The same number of pigs should also be sufficient to demonstrate a 0.20 absolute change in the secondary outcome carotid flow, with a power of 0.80 and SD 0.20.

Mid-sequence intervals from standard mechanical chest compressions were compared with the same intervals from the mechanical ACD-CPR sequences. Two-sided paired samples t-test was used for continuous parametric data and Wilcoxon test for non-parametric data. $\mathrm{P}<0.05$ was considered statistical significant. Analyses were performed using SPSS (version 23 and 24). 


\section{Results}

\section{Paper I}

A total of 4231 patients were included in the CIRC trial and 1657 of them (39.4\%) received one or more shocks during resuscitation for a total of 5336 shocks. In the first paper investigating the predictive value of the one minute post-shock rhythm analysis in relation to the three-minute post-shock rhythm assessment, 43 patients were excluded due to data import failure, missing TTI and/or ECG data, or undeterminable initial cardiac rhythm. This left 1614 patients who received a total of 4867 defibrillator shocks. Of these shocks, 1458 (30\%) were excluded due to data loss one minute and/or three-minute post-shock (missing necessary parts of TTI and/or ECG signal), ICD shocks in-between rhythm assessments, undeterminable cardiac rhythm, inappropriate shocks (shocks delivered to non-shockable rhythms) or too short chest compression pause in relation to rhythm analysis. This left 3409 shocks for analysis, of which 1880 had non-shockable cardiac rhythms one minute post-shock.

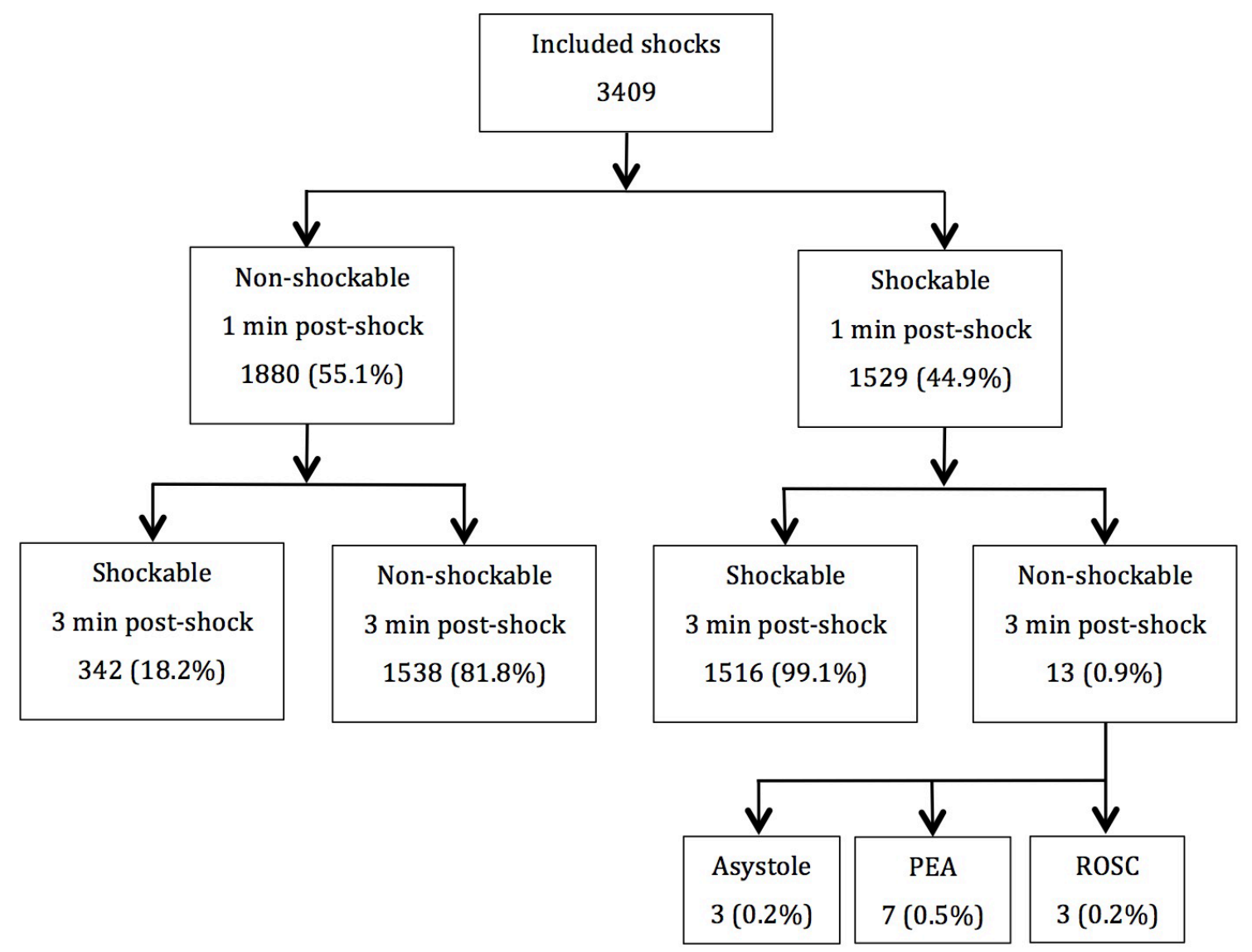

Figure 9. Cardiac rhythm 1 min and 3 min post-shock for the 1614 included patients. Only chest compressions (manual or $L D B-C P R$ ) were delivered between these two rhythm analyses, no defibrillation attempts. 
The rhythm converted to a shockable rhythm three minutes post-shock in 342 cases $(18.2 \%)$ without defibrillation. For the 1529 shocks with a preceding shockable rhythm one minute after the shock, $1516(99.1 \%)$ continued to have a shockable cardiac rhythm two minutes later. In the remaining 13 cases $(0.9 \%)$, the rhythm had converted to a non-shockable rhythm two minutes later without defibrillation; three of these cases resulted in ROSC. Details regarding cardiac rhythm changes one and three minutes after shock are displayed in Figure 9.

CIRC patients received manual or LDB-CPR during resuscitation. When studying the manual CPR and LDB-CPR groups separately, a shockable rhythm one minute post-shock stayed shockable three minutes post-shock in $99.4 \%$ of the time when manual CPR were delivered between rhythm analyses and $98.8 \%$ of the time for LDB-CPR. When the cardiac rhythm converted without defibrillation between one and three minutes post-shock the rhythm converted to asystole in three instances (two after manual CPR and one after LDB-CPR), PEA in seven instances (three after manual CPR and four after LDB-CPR) and ROSC in three instances (one after manual CPR and two after LDB-CPR).

Among the 5336 shocks delivered to CIRC patients 370 (6.9\%) were inappropriately delivered to non-shockable rhythms, among these shocks 148 (40\%) were delivered to organized rhythms (PEA or ROSC) and $222(60 \%)$ to asystole. We had analysable ECG data immediately post-shock for 66 of the 148 shocks delivered to organized rhythms. These 66 shocks were, based on TTI signals and ambulance record, all delivered to PEA and no inappropriate shocks were delivered to perfusing rhythms (ROSC). Among these 66 analysable shocks delivered to PEA the post-shock rhythms remained PEA in 61/66 instances $(92.4 \%)$, converted to asystole in four instances $(6.1 \%)$ and to VF in one instance $(1.5 \%)$.

\section{Paper II}

In the second paper we assessed the relationship between defibrillation success related to which phase of the chest compression cycle shocks were delivered. Among 1657 CIRC patients receiving one or more shocks, 916 had an initial shockable rhythm. Among these, 370 received LDB-CPR prior to shock with a total of 1249 shocks. Figure 10. displays the exclusion criteria's and how the 685 (in the up to three shocks group) and the 224 shocks (in the first shock groups) are distributed as controls or to the different chest compression cycle phases. 


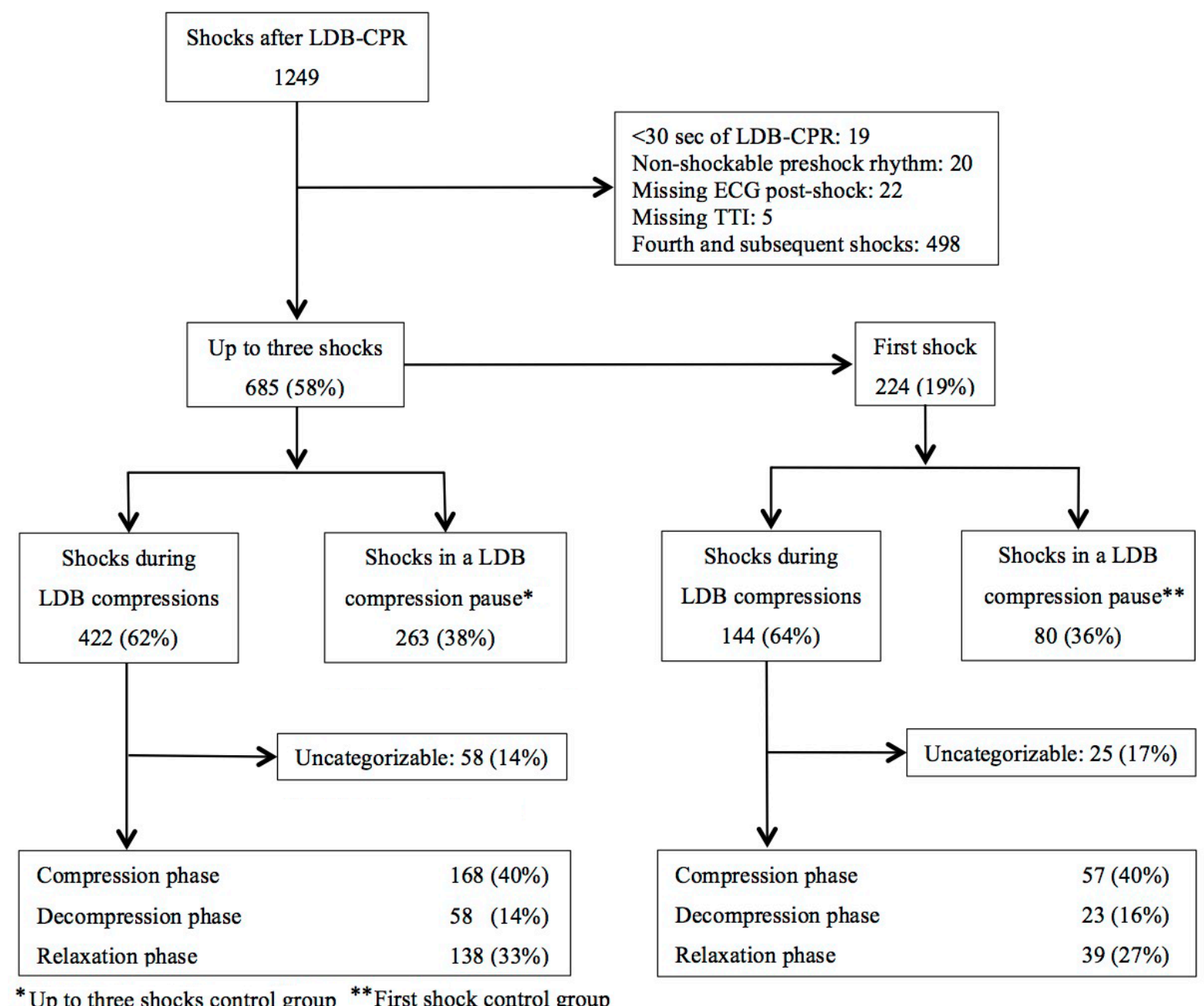

Figure 10. The distribution of shocks included from the 370 patients with initial VF/VT and $L D B-C P R$ prior to shocks.

Shock characteristics for both chest compression cycle phases and controls are presented in table 1. Comparisons of TOF rates between the different phases of the LDB chest compression cycle and controls are presented in table 2 and show significant lower TOF rates when the shock was delivered in the compression phase for both the first shock (14\%) and the up-tothree shocks (11\%). TOF rates were not significantly different between the decompression and relaxation phase and controls for both first shock and up-to-three shocks. Inter-rater agreement assessments of post-shock rhythm annotations resulted in a Kappa value of 0.87 (95 \% CI, $0.84-0.90, p<0.001)$. The assessment of annotation of shocks to LDB chest compression phases resulted in a Kappa value of 0.93 (95\% CI, 0.86-1.00, p<0.001). 
Table 1. Shock characteristics presented as medians and quartiles for controls and compression cycle phases for both first shock and up-to-three shocks analyses.

\begin{tabular}{|l|c|c|c|c|}
\hline First shock group & $\begin{array}{c}\text { Control } \\
\mathrm{n}=80\end{array}$ & $\begin{array}{c}\text { Compression } \\
\mathrm{n}=57\end{array}$ & $\begin{array}{c}\text { Decompression } \\
\mathrm{n}=23\end{array}$ & $\begin{array}{c}\text { Relaxation } \\
\mathrm{n}=39\end{array}$ \\
\hline Joules & $360,200-360$ & $360,200-360$ & $200,200-360$ & $360,200-360$ \\
\hline Transthoracic impedance & $86,67-104$ & $77,65-106$ & $91,71-103$ & $78,62-98$ \\
\hline $\begin{array}{l}\text { Witessed arrest } \\
(\text { missing } \mathrm{n}=9)\end{array}$ & $58(73 \%)$ & $36(63 \%)$ & $20(87 \%)$ & $27(69 \%)$ \\
\hline $\begin{array}{l}\text { Bystander CPR } \\
\text { (missing } \mathrm{n}=8)\end{array}$ & $47(59 \%)$ & $28(49 \%)$ & $16(70 \%)$ & $23(59 \%)$ \\
\hline Up-to-three shocks group & $\begin{array}{c}\text { Control } \\
\mathrm{n}=263\end{array}$ & $\begin{array}{c}\text { Compression } \\
\mathrm{n}=168\end{array}$ & $\begin{array}{c}\text { Decompression } \\
\mathrm{n}=58\end{array}$ & $\begin{array}{c}\text { Relaxation } \\
\mathrm{n}=138\end{array}$ \\
\hline Joules & $360,200-360$ & $360,200-360$ & $360,200-360$ & $360,360-360$ \\
\hline Transthoracic impedance & $85,69-105$ & $80,69-106$ & $90,69-106$ & $84,67-100$ \\
\hline
\end{tabular}

Transthoracic impedance was measured by the defibrillator before shock and not necesarry in the spesific chest compression cycle phase as indicated in this table. The impedance value is however the value used for defibrillator shock energy calculations for the shocks delivered in these specific chest compression cycle phases.

Ambulance records and TTI graph data showed that unintentionally disruptions in the LDB device led to abruptions in chest compressions post-shock in $31 \%$ of shocks delivered during continuous chest compression in the up-to-three shocks group $(n=422)$. These interruptions were associated with shocks delivered in the relaxation phase of the LDB chest compression cycle (compression $17 \%$, decompression $16 \%$, relaxation $49 \%$, p<0.001).

Table 2. TOF rate comparisons presented as unadjusted and adjusted results. First shock analysis utilises logistic regression, up-to-three shock analysis use the GEE model to account for multiple observations per patient.

\begin{tabular}{|c|c|c|c|c|c|}
\hline First shock & TOF (\%) & $\begin{array}{c}\text { Unadjusted } \\
\text { OR (95\% CI) }\end{array}$ & P-value & $\begin{array}{c}\text { Adjusted } \\
\text { OR }(95 \% \text { CI) }\end{array}$ & P-value \\
\hline Control group & $69 / 80(86)$ & & & & \\
\hline Compression & $41 / 57(72)$ & $0.41(0.17-0.97)$ & 0.04 & $0.42\left(0.17-1.00^{*}\right)$ & $0.05^{* *}$ \\
\hline Decompression & $18 / 23(78)$ & $0.57(0.18-1.87)$ & 0.36 & $0.56(0.17-1.83)$ & 0.34 \\
\hline Relaxation & $29 / 39(74)$ & $0.46(0.18 .1 .20)$ & 0.12 & $0.50(0.19-1.32)$ & 0.16 \\
\hline Up to three shocks & TOF (\%) & $\begin{array}{c}\text { Unadjusted } \\
\text { OR (95\% CI) }\end{array}$ & P-value & $\begin{array}{c}\text { Adjusted } \\
\text { OR }(95 \% \mathrm{CI})\end{array}$ & P-value \\
\hline Control group & $216 / 263(82)$ & & & & \\
\hline Compression & $119 / 168(71)$ & $0.55(0.34-0.89)$ & 0.02 & $0.56(0.34-0.91)$ & 0.02 \\
\hline Decompression & $45 / 58(78)$ & $0.70(0.35-1.39)$ & 0.31 & $0.71(0.36-1.39)$ & 0.32 \\
\hline Relaxation & $103 / 138(75)$ & $0.67(0.40-1.14)$ & 0.14 & $0.66(0.38-1.13)$ & 0.13 \\
\hline
\end{tabular}

*Exact OR 95\% CI upper limit $=0.995$

**Exact $\mathrm{p}=0.049$ 


\section{Paper III}

Cardiac output and carotid and cerebral blood flows were significantly higher during ACDCPR. No significant differences were seen in mean aortic, right atrial, CPP, oesophageal or intracranial pressures, nor in $\mathrm{EtCO}_{2}$ or arterial and venous blood gases.

When analysing haemodynamic effects during the different phases of the chest compression cycle (see figure 11.), aortic pressure was significantly higher in the peak compression phase (equivalent to systole), CPP trended towards higher values in the late decompression phase (equivalent to late diastole) and ICP was significantly lower in the end decompression phase for ACD-CPR vs. regular mechanical CPR. Cerebral flow was significantly higher during all chest compression phases of ACD-CPR, while carotid flow, right atrial and oesophageal pressures were not different during the different phases of the chest compression cycle.

A combination of device failure and injuries during the experiment warranted an addition of 10 pigs to conclude the study. The following reasons led to exclusions of pigs: a sternum fixation screw punctured the heart $(n=1)$, sternal fracture and puncture of the right atrium $(n=1)$, breaking of sternal plate $(n=1)$, loosening of sternal screws and plate $(n=2)$, compression device failure $(n=2)$, loss of cardiac output-values and/or large thoracic bleeding $(n=3)$. 
Table 3. Comparison between standard mechanical CPR and ACD-CPR. Continuous parametric data are presented with means and mean difference, non-parametric data are presented as medians and quartiles.

\begin{tabular}{|c|c|c|c|c|c|c|}
\hline & $\begin{array}{c}\text { Standard CPR } \\
\text { mean } \pm \text { SD }\end{array}$ & $\begin{array}{l}\text { ACD-CPR } \\
\text { mean } \pm \text { SD }\end{array}$ & $\begin{array}{l}\text { Mean difference } \\
(95 \% \mathrm{CI})\end{array}$ & $\begin{array}{c}\text { Standard CPR } \\
\text { median } \\
\text { (quartiles) }\end{array}$ & $\begin{array}{l}\text { ACD-CPR } \\
\text { median } \\
\text { (quartiles) }\end{array}$ & p-value \\
\hline \multicolumn{7}{|l|}{ Blood pressures (mmHg) } \\
\hline Aortic pressure & & & & $55(51,70)$ & $60(51,70)$ & 0.86 \\
\hline Right atrial pressure & $54 \pm 16$ & $55 \pm 16$ & $1.0(-4.0,6.0)$ & & & 0.66 \\
\hline Coronary perfusion pressure & $2.2 \pm 22$ & $0.6 \pm 20$ & $-2.7(-10,4.7)$ & & & 0.42 \\
\hline Oesophageal pressure & $57 \pm 38$ & $60 \pm 39$ & $2.6(-2.6,7.8)$ & & & 0.28 \\
\hline Intracranial pressure & $24 \pm 6.4$ & $23 \pm 6.7$ & $-0.7(-1.6,0.1)$ & & & 0.09 \\
\hline \multicolumn{7}{|l|}{ Blood flow } \\
\hline Cerebral flow (AU) & $253 \pm 268$ & $297 \pm 264$ & $44(14,74)$ & & & 0.01 \\
\hline Carotid artery flow (ml/min) & & & & $83(57,94)$ & $97(70,106)$ & $<0.01$ \\
\hline \multicolumn{7}{|l|}{ Manual measurements } \\
\hline Cardiac output (1/min) & & & & $1.1(0.8,1.5)$ & $1.5(1.1,1.7)$ & $<0.01$ \\
\hline End tidal $\mathrm{CO}_{2}(\mathrm{kPa})$ & $2.2 \pm 0.8$ & $2.5 \pm 1.0$ & $0.3(-0.1,0.7)$ & & & 0.15 \\
\hline \multicolumn{7}{|l|}{ Blood gases (kPa) } \\
\hline $\mathrm{pH}$ - Arterial & & & & $7.3(7.2,7.4)$ & $7.3(7.2,7.4)$ & 0.33 \\
\hline $\mathrm{pCO}_{2}$ - Arterial & & & & $5.6(4.9,8.0)$ & $5.4(4.4,8.1)$ & 0.89 \\
\hline $\mathrm{pO}_{2}$ - Arterial & $22 \pm 20$ & $25 \pm 21$ & $2.5(-6.2,11)$ & & & 0.52 \\
\hline $\mathrm{pH}$ - Venous & & & & $7.1(7.1,7.2)$ & $7.2(7,1,7.2)$ & 0.09 \\
\hline $\mathrm{pCO}_{2}$ - Venous & $11 \pm 1.9$ & $11 \pm 1.9$ & $-0.3(-0.5,0.0)$ & & & 0.06 \\
\hline $\mathrm{pO}_{2}$ - Venous & & & & $3.1(3.0,3.6)$ & $3.3(3.1,3.6)$ & 0.11 \\
\hline
\end{tabular}




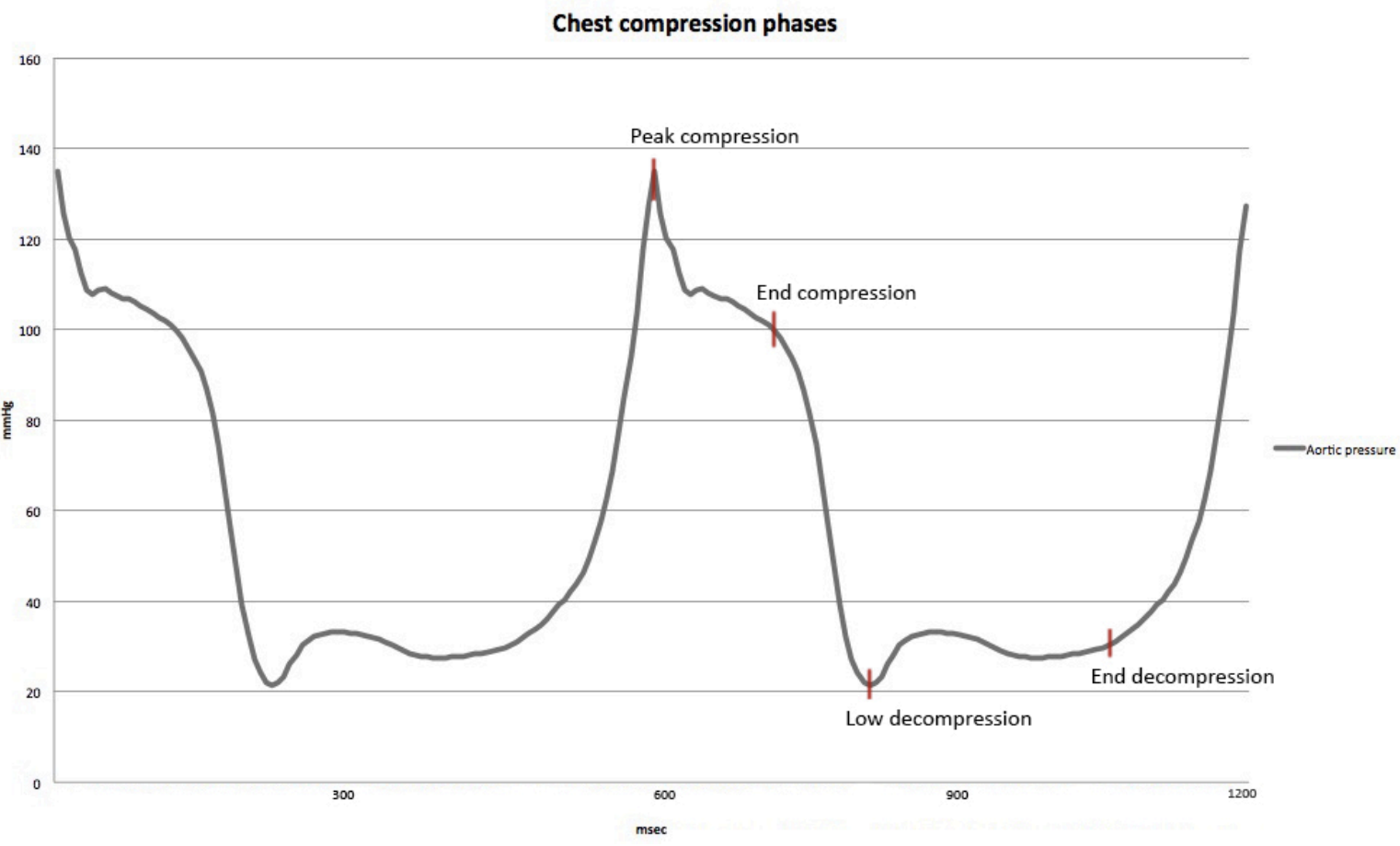

Figure 11. Demonstrates a pressure curve (aortic pressure in this example) and our definitions of the different phases of the chest compression cycle. Chest compression cycle phases was determined based on aortic pressure curves.

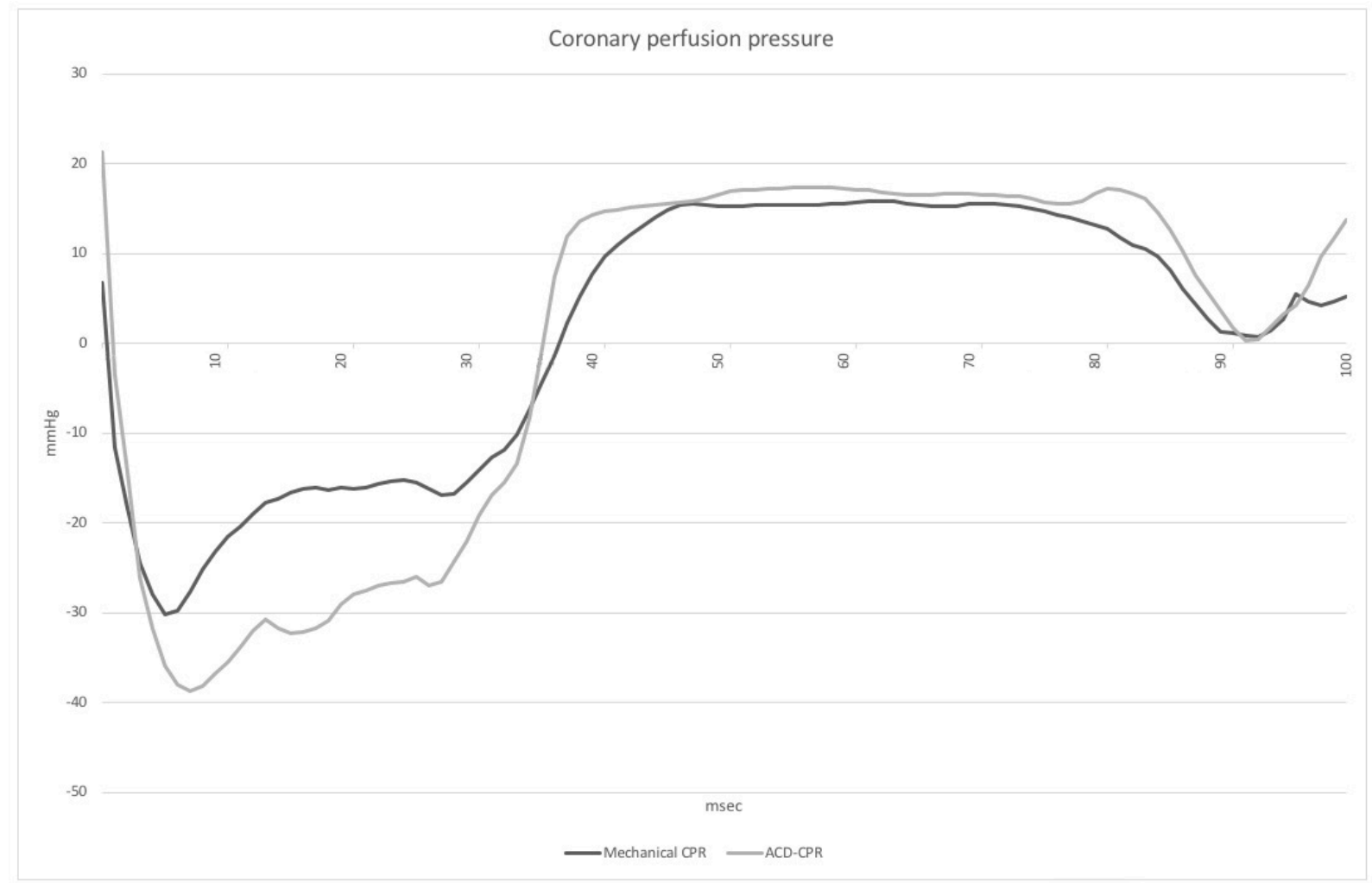

Figure 12. Average pressure curves for both standard mechanical CPR and ACD-CPR demonstrating coronary perfusion pressure. 
Table 4. Standard mechanical CPR compared with ACD-CPR during different chest compression cycle phases.

\begin{tabular}{|c|c|c|c|c|c|c|}
\hline & $\begin{array}{c}\text { Standard CPR } \\
\text { mean } \pm \text { SD }\end{array}$ & $\begin{array}{l}\text { ACD-CPR } \\
\text { mean } \pm \text { SD }\end{array}$ & $\begin{array}{c}\text { Mean difference } \\
(95 \% \mathrm{CI})\end{array}$ & $\begin{array}{c}\text { Standard CPR } \\
\text { median } \\
\text { (quartiles) }\end{array}$ & $\begin{array}{c}\text { ACD-CPR } \\
\text { median } \\
\text { (quartiles) }\end{array}$ & p-value \\
\hline \multicolumn{7}{|l|}{ Blood pressures (mmHg) } \\
\hline \multicolumn{7}{|l|}{ Aortic pressure } \\
\hline Peak compression & $180 \pm 78$ & $196 \pm 79$ & $16(1.1,30)$ & & & 0.04 \\
\hline End compression & $73 \pm 65$ & $68 \pm 85$ & $-4.9(-29,19)$ & & & 0.65 \\
\hline Low decompression & $10 \pm 11$ & $8.7 \pm 13$ & $-1.4(-8.6,5.8)$ & & & 0.66 \\
\hline End decompression & $28 \pm 10$ & $30 \pm 9.1$ & $2.0(-1.1,5.2)$ & & & 0.18 \\
\hline \multicolumn{7}{|l|}{ Right atrial pressure } \\
\hline Peak compression & $172 \pm 71$ & $180 \pm 75$ & $7.8(-17,33)$ & & & 0.49 \\
\hline End compression & $91 \pm 31$ & $97 \pm 32$ & $6.3(-3.2,16)$ & & & 0.16 \\
\hline Low decompression & & & & $7.7(5.5,22)$ & $13(5.6,21)$ & 0.59 \\
\hline End decompression & & & & $11(11,18)$ & $11(9.4,17)$ & 0.11 \\
\hline \multicolumn{7}{|l|}{ Coronary perfusion pressure } \\
\hline Peak compression & $8.3 \pm 39$ & $16 \pm 44$ & $7.8(-9.8,25)$ & & & 0.34 \\
\hline End compression & & & & $-21(-39,6.0)$ & $-19(-39,6.5)$ & 0.59 \\
\hline Low decompression & $-1.2 \pm 14$ & $-6.2 \pm 17$ & $-4.9(-11,1.4)$ & & & 0.11 \\
\hline End decompression & $12 \pm 11$ & $16 \pm 9.4$ & $4.3(-0.2,8.7)$ & & & 0.06 \\
\hline \multicolumn{7}{|l|}{ Intracranial pressure } \\
\hline Peak compression & & & & $38(28,44)$ & $41(24,45)$ & 0.37 \\
\hline End compression & $35 \pm 10$ & $35 \pm 12$ & $-0.2(-4.2,3.8)$ & & & 0.91 \\
\hline Low decompression & $23 \pm 8.3$ & $24 \pm 6.7$ & $1.4(-2.1,4.8)$ & & & 0.39 \\
\hline End decompression & $15 \pm 4.9$ & $14 \pm 5.2$ & $-0.7(-1.2,-0.1)$ & & & 0.03 \\
\hline \multicolumn{7}{|l|}{ Oesophageal pressure } \\
\hline Peak compression & $106 \pm 83$ & $118 \pm 94$ & $13(-17,42)$ & & & 0.35 \\
\hline End compression & $76 \pm 48$ & $84 \pm 51$ & $7.4(-3.3,18)$ & & & 0.15 \\
\hline Low decompression & $40 \pm 37$ & $40 \pm 34$ & $-0.4(-12,11)$ & & & 0.94 \\
\hline End decompression & $39 \pm 29$ & $39 \pm 32$ & $0.0(-7.8,7.7)$ & & & 0.99 \\
\hline \multicolumn{7}{|l|}{ Blood flow } \\
\hline \multicolumn{7}{|l|}{ Cerebral flow (AU) } \\
\hline Peak compression & $248 \pm 268$ & $285 \pm 261$ & $38(9.8,66)$ & & & 0.01 \\
\hline End compression & $244 \pm 255$ & $278 \pm 251$ & $34(8.7,60)$ & & & 0.02 \\
\hline Low decompression & $250 \pm 257$ & $288 \pm 254$ & $38(5.3,70)$ & & & 0.03 \\
\hline End decompression & $266 \pm 283$ & $316 \pm 283$ & $51(18,82)$ & & & $<0.01$ \\
\hline \multicolumn{7}{|l|}{ Carotid artery flow $(\mathrm{ml} / \mathrm{min})$} \\
\hline Peak compression & $288 \pm 93$ & $311 \pm 106$ & $23(-16,62)$ & & & 0.21 \\
\hline End compression & & & & $159(59,321)$ & $176(100,223)$ & 0.17 \\
\hline Low decompression & & & & $7.7(-99,51)$ & $17(-47,97)$ & 0.37 \\
\hline End decompression & & & & $22(-19,98)$ & $38(-7.7,117)$ & 0.09 \\
\hline
\end{tabular}




\section{Discussion}

The three studies focus on different chest compressions methods and their relationship with defibrillation and haemodynamics, with special attention to the timing of cardiac rhythm analysis and defibrillation. Investigations were made to explore and try to understand how we can organize the ALS cycle as effective as possible in order to keep chest compression pauses to a minimum. This might affect the direct outcome of defibrillation attempts, with the overall goal to improve short- and long-term outcome for the patient. Our results indicate that avoiding late rhythm analysis if a shockable rhythm previously has been detected might be problematic. Further, that defibrillation during continuous mechanical chest compressions seems unfortunate for short-term defibrillation outcome. And finally, that a clinical usable mechanical ACD-CPR device generated better haemodynamic outcomes than standard mechanical chest compressions in an experimental pig model. In the following part I will discuss these results up against current available literature and current guidelines.

\section{Paper I}

\section{Chest compression pauses and inappropriate shocks}

A shockable rhythm one minute after a shock was highly predictive of still being shockable two minutes later. However, in three instances the rhythm converted to a perfusing rhythm sometime during the two minutes of chest compressions following the treatment protocol. Consequently, if the immediate pre-shock rhythm analysis at three minutes is removed from the local ALS protocol in the presence of a shockable rhythm already confirmed one minute after the shock, we might pose the risk of shocking a patient with a perfusing rhythm. Even though the risk was low, consequences of shock delivery to a patient with a perfusing rhythm could be detrimental. A shock delivered around the ECG T-wave could in worst case result in re-arrest with $\operatorname{VF}(217,218)$ or VT.(219) Fortunately, due to the ALS protocol-based required reassessment of the rhythm analysis prior to shock, none of these three patients from the CIRC trial were shocked. We did, however, see that $6.9 \%$ of all defibrillation attempts in the CIRC material were delivered to non-shockable rhythms. Among these inappropriate shocks, the majority $(96.3 \%)$ did not alter the cardiac rhythm. Kramer-Johansen et al showed the same trend for 193 inappropriate shocks whereof 150 were given during organized rhythms.(220) In 12 cases, organized rhythms changed as a result of defibrillation, these were all episodes of PEA and no circulation was detected by TTI. (220) Based on this low risk of harm, it could be tempting to advice against pausing chest compressions to re-analyse the cardiac rhythm if 
VF/VT have already been verified earlier in the CPR cycle. It is however difficult to quantify the positive effect of such an approach and weigh this against the risk of inadvertently shocking a perfusing rhythm into a new cardiac arrest. Further, among patients with a nonshockable rhythm one minute after shock, $18.2 \%$ converted to VF/VT after chest compressions. The predictable value for a non-shockable rhythm one minute after a shock is therefore too poor to enable the removal of a second rhythm check for non-shockable rhythms. This would result in a significant number of shockable rhythms being overseen with missed opportunities to defibrillate.

In one previous study, shorter pre-shock chest compression pauses were associated with increased first shock TOF rate.(123) This study had major impact on the discussion on preshock pauses and defibrillation, but it has been criticized for its low number of patients $(n=60)$. Olsen et al found rather surprisingly that the first shock TOF rate was lower for shocks delivered during chest compressions (ie. zero pre-shock pause).(175) Both increased CCF(127) and reduced pre-shock chest compression pauses(126) have in other studies been associated with increased ROSC. Cheskes et al found that shorter pre-shock chest compression pauses were associated with higher survival rate, but no patient had zero pre-shock pause in their studies. $(128,129)$ When pre-shock chest compression pauses decrease, CCF will increase. The survival benefit of reduced chest compression pauses immediately pre-shock could therefore be a proxy for the survival benefit of increased CCF.(130) One study has however shown that the chest compressions pause immediately pre-shock is an independent predictor for survival after adjusting for CCF.(129)

Eliminating the rhythm analysis immediately pre-shock if VF/VT is already confirmed one minute post-shock would reduce chest compressions pauses immediately pre-shock. The rhythm analysis would however have to take place at some point, though earlier. This approach would therefore not increase CCF during the standard two-minute CPR cycle recommended by both ERC and AHA, where only one routine rhythm check is advised.(70, 71) Such an approach could only improve survival if the chest compressions pause immediately pre-shock were an independent predictor for survival after adjusting for CCF, and this has, as previously mentioned, only been found in one study so far.(129) The Norwegian CPR guidelines do however recommend a three-minute CPR cycle with a short rhythm check both one and three minutes post-shock.(121) Eliminating the analysis at three minutes would reduce pre-shock chest compression pauses, increase CCF and theoretically impact on patient outcome. However, this has to be investigated in a prospectively designed study. 
The risk of shocking a perfusing rhythm could be minimized if there was a way to determine ROSC during chest compressions. EtCO ${ }_{2}$ values are usually much lower during CPR (0-2.7 $\mathrm{kPa})(221)$ than during normal circulation and ventilation $(4.6-5.9 \mathrm{kPa}) \cdot(222,223)$, and it has been reported that $\mathrm{EtCO}_{2}$ measured during CPR increases suddenly when ROSC is achieved.(224) Before this method can be used to ensure avoidance of shocking a perfusing rhythm, a thorough analysis of predictive value in a much larger patient cohorts is required, including good data analysis on how fast $\mathrm{EtCO}_{2}$ increases after ROSC. EtCO2 values are also influenced by a number of cofounding factors, including cause of cardiac arrest, initial rhythm, time from cardiac arrest and bystander CPR, complicating the interpretation of capnography during resuscitation.(225)

Pulse generating blood flow is known to generate changes in TTI in pigs(226) and has been used to verify ROSC in clinical trials(227) and on human datasets, with a sensitivity of 94 \%.(228) These TTI evaluations have however been conducted during chest compression pauses with an initially flat TTI curve, and TTI has to my knowledge not been used to verify ROSC during chest compressions. This would require filtering out TTI signal changes from chest compressions without affecting the TTI signals from the spontaneous circulation.

\section{Rhythm conversion without defibrillation}

Spontaneous rhythm conversion has been described in both healthy adults $(229,230)$ and in patients with myocardial infarction. $(231,232)$ Sunde et al have demonstrated that rhythm conversion frequently can happen even more than 30 seconds after defibrillation in OHCA patients,(233) and this was thought to be a result of myocardial stunning after defibrillation.(234) Both of these factors might contribute to our findings of shockable rhythms converting to both PEA and ROSC without defibrillation/or very late after a previous defibrillation during chest compressions. It is also possible that VF/VT was converted by the chest compressions, which can stimulate the heart electrically. $(235,236)$ Drug management might also have contributed to these rhythm conversions. CIRC patients could receive amiodarone, lidocaine, atropine, bicarbonate, adrenaline and/or vasopressin based on EMS sites and their routines.(168) Although drug use was noted in the case report forms, the timing of drug delivery was not noted, and we do therefore not know if drugs were administrated in association with these episodes with rhythm conversion. In conclusion, there are too many uncertainties in the current material to conclude either way regarding the reason for these rhythm conversions. 


\section{Limitations}

Paper I is based on retrospective analysis of CIRC data, and not data prospectively collected for the purpose of the study. A RCT comparing two CPR cycle strategies would be the preferred design to further study this.

The CIRC trial was carried out following the Norwegian CPR cycle recommendation of three minutes.(168) This makes the results from the present study not directly transferable to EMS services following ERC/AHA CPR guidelines with a two minute CPR cycle and one rhythm analysis per cycle.

The large number of excluded shocks is also a limitation. This was necessary in order to analyse an artefact-free ECG at two times during the CPR cycle. Protocol violation with lack of a chest compression pause for the one minute rhythm analysis was the most common reason for exclusion.

\section{Paper II}

\section{Defibrillation success during chest compressions}

TOF rate was lower when a shock was delivered in the compression phase of the compressiondecompression cycle compared to shocks not delivered during chest compressions. The only previous comparable studies were carried out by Li et al in pigs.(176, 177) They studied shocks delivered during both manual and mechanical chest compressions in five different phases of the compression-decompression cycle and compared these with shocks delivered after a two second chest compressions pause (controls). The methods were somewhat similar in the two studies, but ours were retrospective in nature and the pause before our control shocks represented the actual pre-shock pauses for included shocks from the CIRC trial (median $12 \mathrm{sec}$ for first shocks and $13 \mathrm{sec}$ for the up-to-three first shocks). Li et al found that TOF was higher in the upstroke phase (equal to our decompression phase) for both manual and mechanical chest compressions. $(176,177)$ Shocks delivered immediately before the compression phase (equal to our late decompression phase) of manual chest compressions had lower TOF rates than controls(176) With mechanical chest compressions there was no such difference.(177) We failed to demonstrate any favourable chest compression phase for shock delivery compared with controls, but found significantly lower TOF rates for shocks delivered in the compression phase compared with controls. Our results do not necessary oppose the results by Li et al, but also indicate that the timing of shock delivery during continuous chest compressions might be important for TOF. 


\section{Why defibrillation during chest compression yields different results?}

A possible explanation for the low TOF rates for shocks delivered in the compression phase of LDB-CPR might be due to chest compression generated geometrical changes to the heart and nearby vessels. Trans-oesophageal echocardiography has demonstrated both distortion of the great vessels and reduction in size of all cardiac chambers during chest compressions.(237) During defibrillation, a large electrical current is sent between the defibrillator pads. The two hypotheses behind how defibrillation works are "the critical mass theory"(238) and "the upper limit of vulnerability theory".(239) The critical mass theory hypothesise that a critical mass of excitable cells in the myocardium has to be depolarized simultaneously in order to achieve defibrillation.(238) The upper limit of vulnerability theory describes that not only excitable cells in the myocardium have to be depolarized in order to achieve successful defibrillation, but also myocardial cells in their relative refractory period. To achieve this, the electronic stimulus must exceed the upper limit of vulnerability, which is the same stimulus strength that can induce fibrillation in a heart during the vulnerable period of the repolarization phase around the T-wave.(239) It has been demonstrated that only approximately $4 \%$ of the current sent between two defibrillator pads actually pass through the myocardium with anterior lateral pads placement.(240) The geometrical changes to the heart and its changed position inside the thoracic cavity during chest compressions could potentially influence the amount of current reaching the myocardium, and thus also defibrillation success. A change in angle of the defibrillator pads during chest compressions could also influence the amount of current available for myocardial depolarization, and thereby defibrillation success. This is indeed only free speculations, and electrophysiological studies of shock delivery during chest compressions are necessary in order to gain further knowledge on this matter.

A second possible explanation for lower TOF rates in the compression phase is based on the geometric changes in thorax during chest compressions and how this influence TTI. Li et al found that TTI increased slightly during both manual and mechanical chest compressions.(176, 177) The LIFEPAK ${ }^{\circledR} 12 / 15$ defibrillators used in the CIRC trial have been reported by the manufacturer to measure TTI at a time interval before shock is delivered. If the TTI changes significantly from the time of TTI measurement to when the shock is actually delivered, this could affect the current and voltage levels of the shock, and thereby potentially defibrillation success. Li et al did however not find this association. $(176,177)$ They discovered that the shock-induced tetanic body contraction of the animal combined with the force from manual chest compressions almost doubled in the compression phase of the chest compression cycle.(176) This physical strain might possibly affect myocytes in a way that makes them less susceptible to defibrillation, but no force measurements were made in the CIRC trial and to my 
knowledge a possible association between physical strain on the heart and defibrillation success has not been studied.

\section{Limitations}

Paper II is based on retrospective analysis of CIRC data, and not data prospectively collected for the purpose of the study. A RCT comparing different defibrillation strategies would be the preferred design for a prospective study.

The large number of excluded shocks is also a limitation. We were unable to assign a LDB compression cycle phase to a high number of shocks (17\% first shock, $14 \%$ up-to-three shocks group), but we have no reason to believe this was anything but random. There is still, however, the possibility of bias.

The method used to retrospectively determine the timing of shocks delivered during chest compressions is newly developed and have to my knowledge not been used before. The kappa score for inter-rater agreement analysis was however high, indicating excellent agreement in shock assignment.

Statistical analyses in the present study were not adjusted for multiple comparisons. Even though the possibility of a type I error is present, adjustments for multiple comparisons would increase the risk for type II errors. $(241,242)$

Further, first shock observations were based on independent patient observations, but in the up-to-three shocks group one patient could contribute shocks to several different LDB phases. Adjustments using the GEE model were made in the statistical analyses in order to account for multiple observations per patient.

Finally, the present results are based on the LDB device and are therefore not directly transferable to other mechanical chest compression devices. Since the LDB device compresses the chest with a circumferential band, this might change both anatomical structures in the chest and the angle of the defibrillator pads differently from a piston-based device.

\section{Paper III}

\section{Haemodynamic benefits of ACD-CPR}

Active decompression should in theory improve vital perfusion by lowering the intrathoracic pressure in the decompression phase of the chest compression cycle. This is thought to 
increase the filling of the ventricles, and thus increase both stroke volume and cardiac output. The present results, with increased cardiac output as well as carotid and cerebral blood flow, confirm findings from previous studies on dogs. $(180,183)$ Haemodynamic benefits of ACDCPR have also been reported in humans. $(187,188)$ All these earlier studies utilised a handheld ACD-CPR device, but similar results have been found with a power driven mechanical piston based chest compression device.(181) Although previous studies demonstrated increases in blood pressures, $(180,183-185)$ we were unable to demonstrate significant increases in mean pressures with ACD-CPR. However, there was a significant increase in aortic blood pressure during peak compression (equivalent to systole), and CPP trended towards higher values during end decompression (equivalent to late diastole). CPP can be reported in a number of different ways, and this poses a relevant problem when comparing different studies. Otlewski et al concluded that true mean CPP values (including the whole chest compression cycle) are the most reliable comparator between studies.(243) In the present study, true mean CPP was measured and we subdivided the chest compression cycle into different phases. Negative CPP during the compression phase is similar to previous findings using a very similar methodology, but with a stationary piston based mechanical chest compression device.(184) This agrees with findings in earlier studies indicating that myocardial perfusion only takes place during the decompression phase (diastole) of the chest compression cycle. $(183,185,244,245)$ This is probably due to the relationship between CPP and myocardial perfusion in a low pressure scenario such as CPR, where myocardial perfusion is directly related to CPP differing from a normal circulatory state where other factors are more important.(246) Thus, increased aortic pressure and/or decreased right atrial pressure with increased CPP during decompression should therefore potentially improve myocardial perfusion during CPR.(247) We were unable to demonstrate decreased oesophageal pressure as a proxy for intrathoracic pressure or a decrease in right atrial pressure during decompression. In one study, ACD-CPR resulted in a decrease in oesophageal pressure, but without a reduction in right atrial pressure.(185)

A few studies on ACD-CPR and haemodynamic effects on animals utilised mechanical chest compression devices capable of delivering active decompression. These devices were however large and stationary, not suited for cardiac arrest research on humans.(181, 184, 185, 248) All studies on the effects of ACD-CPR in humans have therefore to my knowledge been carried out using a manual, handheld device. Animal studies utilising this manual device demonstrated similar effects on blood pressures, cardiac output and organ blood flow as the mechanical devices.(180, 182, 183) It would be interesting to see if the haemodynamic benefits of mechanical ACD-CPR indicated in paper III, also occured in humans with the potential for improved final outcome. 


\section{Limitations}

Although earlier studies reported similar haemodynamic effects of ACD-CPR in pigs and humans, the results in Paper III are not directly transferable to humans. The cross-over nature of the study design results in a possible carry-over effect from control to intervention or vice versa. However, the three-phase design of the study does take this into account and should at least partly make this kind of bias less likely by repeating the first modality.

A high number of pigs were excluded due to a combination of procedural failures during instrumentation, material or device failure during the experimental phase and injuries. In the clinical version of the LUCAS device, the piston is fastened to the chest by a suction cup. We have in earlier experimental models experienced difficulties fastening suctions cups to pig chests, which have a different configuration than in humans, and therefore chose to fasten the piston device directly to a metal plate on the pigs sternum. Four of ten excluded pigs were due to problems with the sternal screws and/or plate and are therefore not applicable for the suction cup used in humans. Moreover, two exclusions were due to chest compression device failure, which can be attributed to the fact that we used a first time ACD-modification of a chest compression device originally developed without this capability. The remaining four exclusions were due to injuries with rupture of large vessels, cardiac tamponade, punctured right atrium and one episode of sternal fracture. We hypothesise that these serious injuries resulted from the very direct transfer of forces occurring when the chest compression piston is fastened directly to the sternum. These forces are especially high during the transition between compression and decompression and a suction cup will probably absorb a high amount of these forces.

Further, there is also a significant anatomical difference between pigs and humans that might be relevant. A pig's pericardium adheres directly to the inside of the sternum, drag forces applied to the sternum thus might make a pig heart more susceptible to injury.(249) Potential different injury profiles between standard mechanical CPR and ACD-CPR in the present study could not be studied because of the crossover study design where each pig served as its own control. Two post-mortem studies of clinical ACD-CPR reported higher incidence of rib and sternal fractures after ACD-CPR, $(250,251)$ but a later review found no compelling evidence that ACD-CPR increases complication rates.(252) Again, it should be noted that the mentioned studies used the handheld ACD-CPR device, different from our mechanical approach in paper III. 


\section{Conclusions}

The predictive value of a shockable rhythm one minute after a shock was very high for a continued shockable rhythm three minutes post-shock, but three patients regained a perfusing rhythm between the two rhythm analyses. More research is needed to study ROSC detection during chest compressions and whether the immediate pre-shock chest compression pause is a predictor of survival independent of the chest compression fraction

The short-term electrical outcome defibrillation success was significantly lower when defibrillation occurred during the compression phase of the compression-decompression cycle utilising a load-distributing band chest compression device in humans, compared with shocks delivered during a pause in chest compressions. The mechanism behind these results is not clear and need further investigation.

Mechanical ACD-CPR delivered by a software modified clinically available chest compression device with five $\mathrm{cm}$ of compression combined with two $\mathrm{cm}$ of active decompression above the resting chest level resulted in higher cardiac output, cerebral and carotid blood flow in an experimental pig model, compared to standard mechanical chest compressions. Clinical studies on mechanical ACD-CPR would be of interest to determine effects on clinical outcomes in patients. 


\section{References}

1. Global, regional, and national age-sex specific all-cause and cause-specific mortality for 240 causes of death, 1990-2013: a systematic analysis for the Global Burden of Disease Study 2013. Lancet. 2015;385(9963):117-71.

2. WHO. World Health Organization, World Health Statistics 2016.

3. Go AS, Mozaffarian D, Roger VL, Benjamin EJ, Berry JD, Borden WB, et al. Heart disease and stroke statistics--2013 update: a report from the American Heart Association. Circulation. 2013;127(1):e6-e245.

4. Berdowski J, Berg RA, Tijssen JGP, Koster RW. Global incidences of out-of-hospital cardiac arrest and survival rates: Systematic review of 67 prospective studies. Resuscitation. 2010;81(11):1479-87.

5. Cummins RO, Chamberlain DA, Abramson NS, Allen M, Baskett PJ, Becker L, et al. Recommended guidelines for uniform reporting of data from out-of-hospital cardiac arrest: the Utstein Style. A statement for health professionals from a task force of the American Heart Association, the European Resuscitation Council, the Heart and Stroke Foundation of Canada, and the Australian Resuscitation Council. Circulation. 1991;84(2):960-75.

6. Cummins RO, Chamberlain D, Hazinski MF, Nadkarni V, Kloeck W, Kramer E, et al. Recommended guidelines for reviewing, reporting, and conducting research on in-hospital resuscitation: the in-hospital "Utstein style". American Heart Association. Ann Emerg Med. 1997;29(5):650-79.

7. Zaritsky A, Nadkarni V, Hazinski MF, Foltin G, Quan L, Wright J, et al. Recommended guidelines for uniform reporting of pediatric advanced life support: the Pediatric Utstein Style. A statement for healthcare professionals from a task force of the American Academy of Pediatrics, the American Heart Association, and the European Resuscitation Council. Resuscitation. 1995;30(2):95-115.

8. Idris AH, Becker LB, Ornato JP, Hedges JR, Bircher NG, Chandra NC, et al. Utstein-style guidelines for uniform reporting of laboratory CPR research. A statement for healthcare professionals from a Task Force of the American Heart Association, the American College of Emergency Physicians, the American College of Cardiology, the European Resuscitation Council, the Heart and Stroke Foundation of Canada, the Institute of Critical Care Medicine, the Safar Center for Resuscitation Research, and the Society for Academic Emergency Medicine. Resuscitation. 1996;33(1):69-84. 
9. Langhelle A, Nolan J, Herlitz J, Castren M, Wenzel V, Soreide E, et al. Recommended guidelines for reviewing, reporting, and conducting research on post-resuscitation care: the Utstein style. Resuscitation. 2005;66(3):271-83.

10. Jacobs I, Nadkarni V, Bahr J, Berg RA, Billi JE, Bossaert L, et al. Cardiac arrest and cardiopulmonary resuscitation outcome reports: update and simplification of the Utstein templates for resuscitation registries. A statement for healthcare professionals from a task force of the international liaison committee on resuscitation (American Heart Association, European Resuscitation Council, Australian Resuscitation Council, New Zealand Resuscitation Council, Heart and Stroke Foundation of Canada, InterAmerican Heart Foundation, Resuscitation Council of Southern Africa). Resuscitation. 2004;63(3):233-49.

11. Perkins GD, Jacobs IG, Nadkarni VM, Berg RA, Bhanji F, Biarent D, et al. Cardiac arrest and cardiopulmonary resuscitation outcome reports: update of the Utstein Resuscitation Registry Templates for Out-of-Hospital Cardiac Arrest: a statement for healthcare professionals from a task force of the International Liaison Committee on Resuscitation (American Heart Association, European Resuscitation Council, Australian and New Zealand Council on Resuscitation, Heart and Stroke Foundation of Canada, InterAmerican Heart Foundation, Resuscitation Council of Southern Africa, Resuscitation Council of Asia); and the American Heart Association Emergency Cardiovascular Care Committee and the Council on Cardiopulmonary, Critical Care, Perioperative and Resuscitation. Circulation. 2015;132(13):1286-300.

12. Dick WF, Baskett PJ, Grande C, Delooz H, Kloeck W, Lackner C, et al. Recommendations for uniform reporting of data following major trauma--the Utstein style. An International Trauma Anaesthesia and Critical Care Society (ITACCS) initiative. Br J Anaesth. 2000;84(6):818-9.

13. Ersdal HL, Singhal N, Msemo G, Kc A, Data S, Moyo NT, et al. Successful implementation of Helping Babies Survive and Helping Mothers Survive programs-An Utstein formula for newborn and maternal survival. PLoS One. 2017;12(6):e0178073.

14. Lund-Kordahl I, Olasveengen TM, Lorem T, Samdal M, Wik L, Sunde K. Improving outcome after out-of-hospital cardiac arrest by strengthening weak links of the local Chain of Survival; quality of advanced life support and post-resuscitation care. Resuscitation. 2010;81(4):422-6.

15. Wissenberg M, Lippert FK, Folke F, Weeke P, Hansen CM, Christensen EF, et al. Association of national initiatives to improve cardiac arrest management with rates of 
bystander intervention and patient survival after out-of-hospital cardiac arrest. JAMA. 2013;310(13):1377-84.

16. Tjelmeland IN, JE; Kramer-Johansen, J; Andersson, LJ; Bratland, S; Hafstad, AK; Haug, B; Langørgen, J; Larsen, AI; Skogvoll, E; Søreide, E. Annual Report 2016. Norwegian Cardiac Arrest Registry. 2017.

17. Herlitz J. Annual Report 2016. Swedish CPR registry. 2017.

18. Hansen SR, KB; Wissenberg, M; Mortensen, RN; Folke, F; Lippert, FK; Berlac, P; Mikkelsen, S; Hansen, PA; Nielsen, PS; Hendriksen, OM; Torp-Pedersen, C. Annual Report 2016. Danish Cardiac Arrest Registry. 2017.

19. Cobb LA, Werner JA, Trobaugh GB. Sudden cardiac death. I. A decade's experience with out-of-hospital resuscitation. Mod Concepts Cardiovasc Dis. 1980;49(6):31-6.

20. Cummins RO, Ornato JP, Thies WH, Pepe PE. Improving survival from sudden cardiac arrest: the "chain of survival" concept. A statement for health professionals from the Advanced Cardiac Life Support Subcommittee and the Emergency Cardiac Care Committee, American Heart Association. Circulation. 1991;83(5):1832-47.

21. Nolan J, Soar J, Eikeland H. The chain of survival. Resuscitation. 2006;71(3):270-1.

22. Hasselqvist-Ax I, Riva G, Herlitz J, Rosenqvist M, Hollenberg J, Nordberg P, et al. Early Cardiopulmonary Resuscitation in Out-of-Hospital Cardiac Arrest. New England Journal of Medicine. 2015;372(24):2307-15.

23. Kragholm K, Wissenberg M, Mortensen RN, Hansen SM, Malta Hansen C, Thorsteinsson K, et al. Bystander Efforts and 1-Year Outcomes in Out-of-Hospital Cardiac Arrest. N Engl J Med. 2017;376(18):1737-47.

24. Wik L, Steen PA, Bircher NG. Quality of bystander cardiopulmonary resuscitation influences outcome after prehospital cardiac arrest. Resuscitation. 1994;28(3):195-203.

25. Kuisma M, Repo J, Alaspaa A. The incidence of out-of-hospital ventricular fibrillation in Helsinki, Finland, from 1994 to 1999. Lancet. 2001;358(9280):473-4.

26. Cobb LA, Fahrenbruch CE, Olsufka M, Copass MK. Changing incidence of out-of-hospital ventricular fibrillation, 1980-2000. JAMA. 2002;288(23):3008-13.

27. Polentini MS, Pirrallo RG, McGill W. The changing incidence of ventricular fibrillation in Milwaukee, Wisconsin (1992-2002). Prehosp Emerg Care. 2006;10(1):52-60.

28. Keller SP, Halperin HR. Cardiac arrest: the changing incidence of ventricular fibrillation. Curr Treat Options Cardiovasc Med. 2015;17(7):392. 
29. Youngquist ST, Kaji AH, Niemann JT. Beta-blocker use and the changing epidemiology of out-of-hospital cardiac arrest rhythms. Resuscitation. 2008;76(3):376-80.

30. Wang H, Tang W, Ristagno G, Li Y, Sun S, Wang T, et al. The potential mechanisms of reduced incidence of ventricular fibrillation as the presenting rhythm in sudden cardiac arrest. Crit Care Med. 2009;37(1):26-31.

31. Bunch TJ, White RD, Friedman PA, Kottke TE, Wu LA, Packer DL. Trends in treated ventricular fibrillation out-of-hospital cardiac arrest: a 17-year population-based study. Heart Rhythm. 2004;1(3):255-9.

32. Kouwenhoven WB, Jude JR, Knickerbocker GG. Closed-chest cardiac massage. JAMA. 1960;173:1064-7.

33. Rudikoff MT, Maughan WL, Effron M, Freund P, Weisfeldt ML. Mechanisms of blood flow during cardiopulmonary resuscitation. Circulation. 1980;61(2):345-52.

34. Weisfeldt ML, Chandra N. Physiology of cardiopulmonary resuscitation. Annu Rev Med. $1981 ; 32: 435-42$.

35. Olasveengen TM, Samdal M, Steen PA, Wik L, Sunde K. Progressing from initial nonshockable rhythms to a shockable rhythm is associated with improved outcome after out-ofhospital cardiac arrest. Resuscitation. 2009;80(1):24-9.

36. Rajan S, Folke F, Hansen SM, Hansen CM, Kragholm K, Gerds TA, et al. Incidence and survival outcome according to heart rhythm during resuscitation attempt in out-of-hospital cardiac arrest patients with presumed cardiac etiology. Resuscitation. 2017;114:157-63.

37. Sasson C, Rogers MA, Dahl J, Kellermann AL. Predictors of survival from out-of-hospital cardiac arrest: a systematic review and meta-analysis. Circ Cardiovasc Qual Outcomes. 2010;3(1):63-81.

38. Gallagher E, Lombardi G, Gennis P. EFfectiveness of bystander cardiopulmonary resuscitation and survival following out-of-hospital cardiac arrest. JAMA. $1995 ; 274(24): 1922-5$.

39. Hallstrom A, Cobb L, Johnson E, Copass M. Cardiopulmonary Resuscitation by Chest Compression Alone or with Mouth-to-Mouth Ventilation. New England Journal of Medicine. 2000;342(21):1546-53.

40. Mohler MJ, Wendel CS, Mosier J, Itty A, Fain M, Clark L, et al. Cardiocerebral Resuscitation Improves Out-of-Hospital Survival in Older Adults. J Am Geriatr Soc. 2011;59(5):822-6. 
41. Olasveengen TM, Wik L, Steen PA. Standard basic life support vs. continuous chest compressions only in out-of-hospital cardiac arrest. Acta Anaesthesiol Scand. 2008;52(7):914-9.

42. Bobrow BJ, Spaite DW, Berg RA, et al. Chest compression-only cpr by lay rescuers and survival from out-of-hospital cardiac arrest. JAMA. 2010;304(13):1447-54.

43. Rea TD, Fahrenbruch C, Culley L, Donohoe RT, Hambly C, Innes J, et al. CPR with Chest Compression Alone or with Rescue Breathing. New England Journal of Medicine. 2010;363(5):423-33.

44. Panchal AR, Bobrow BJ, Spaite DW, Berg RA, Stolz U, Vadeboncoeur TF, et al. Chest compression-only cardiopulmonary resuscitation performed by lay rescuers for adult out-ofhospital cardiac arrest due to non-cardiac aetiologies. Resuscitation. 2013;84(4):435-9.

45. Kitamura T, Iwami T, Kawamura T, Nagao K, Tanaka H, Nadkarni VM, et al. Conventional and chest-compression-only cardiopulmonary resuscitation by bystanders for children who have out-of-hospital cardiac arrests: a prospective, nationwide, population-based cohort study. Lancet. 2010;375(9723):1347-54.

46. Iwami T, Kawamura T, Hiraide A, Berg RA, Hayashi Y, Nishiuchi T, et al. Effectiveness of bystander-initiated cardiac-only resuscitation for patients with out-of-hospital cardiac arrest. Circulation. 2007;116(25):2900-7.

47. Kitamura T, Iwami T, Kawamura T, Nagao K, Tanaka H, Berg RA, et al. Time-dependent effectiveness of chest compression-only and conventional cardiopulmonary resuscitation for out-of-hospital cardiac arrest of cardiac origin. Resuscitation. 2011;82(1):3-9.

48. Perkins GD, Handley AJ, Koster RW, Castren M, Smyth MA, Olasveengen T, et al. European Resuscitation Council Guidelines for Resuscitation 2015: Section 2. Adult basic life support and automated external defibrillation. Resuscitation. 2015;95:81-99.

49. Kleinman ME, Brennan EE, Goldberger ZD, Swor RA, Terry M, Bobrow BJ, et al. Part 5: Adult Basic Life Support and Cardiopulmonary Resuscitation Quality: 2015 American Heart Association Guidelines Update for Cardiopulmonary Resuscitation and Emergency Cardiovascular Care. Circulation. 2015;132(18 Suppl 2):S414-35.

50. Olasveengen TM, de Caen AR, Mancini ME, Maconochie IK, Aickin R, Atkins DL, et al. 2017 International Consensus on Cardiopulmonary Resuscitation and Emergency Cardiovascular Care Science With Treatment Recommendations Summary. Resuscitation. 2017;121:201-14. 
51. Perkins GD, Olasveengen TM, Maconochie I, Soar J, Wyllie J, Greif R, et al. European Resuscitation Council Guidelines for Resuscitation: 2017 update. Resuscitation. 2018;123:43-50.

52. Monsieurs KG, Nolan JP, Bossaert LL, Greif R, Maconochie IK, Nikolaou NI, et al. European Resuscitation Council Guidelines for Resuscitation 2015: Section 1. Executive summary. Resuscitation. 2015;95:1-80.

53. Neumar RW, Shuster M, Callaway CW, Gent LM, Atkins DL, Bhanji F, et al. Part 1: Executive Summary: 2015 American Heart Association Guidelines Update for Cardiopulmonary Resuscitation and Emergency Cardiovascular Care. Circulation. 2015;132(18 Supp1 2):S315-67.

54. Idris AH, Guffey D, Pepe PE, Brown SP, Brooks SC, Callaway CW, et al. Chest Compression Rates and Survival Following Out-of-Hospital Cardiac Arrest*. Crit Care Med. 2015;43(4):840-8.

55. Idris AH, Guffey D, Aufderheide TP, Brown S, Morrison LJ, Nichols P, et al. Relationship between chest compression rates and outcomes from cardiac arrest. Circulation. 2012;125(24):3004-12.

56. Abella BS, Sandbo N, Vassilatos P, Alvarado JP, O'Hearn N, Wigder HN, et al. Chest compression rates during cardiopulmonary resuscitation are suboptimal: a prospective study during in-hospital cardiac arrest. Circulation. 2005;111(4):428-34.

57. Bohn A, Weber TP, Wecker S, Harding U, Osada N, Van Aken H, et al. The addition of voice prompts to audiovisual feedback and debriefing does not modify CPR quality or outcomes in out of hospital cardiac arrest - A prospective, randomized trial. Resuscitation. 2011;82(3):257-62.

58. Zuercher M, Hilwig RW, Ranger-Moore J, Nysaether J, Nadkarni VM, Berg MD, et al. Leaning during chest compressions impairs cardiac output and left ventricular myocardial blood flow in piglet cardiac arrest. Crit Care Med. 2010;38(4):1141-6.

59. Edelson DP, Litzinger B, Arora V, et al. Improving in-hospital cardiac arrest process and outcomes with performance debriefing. Arch Intern Med. 2008;168(10):1063-9.

60. Babbs CF, Kemeny AE, Quan W, Freeman G. A new paradigm for human resuscitation research using intelligent devices. Resuscitation. 2008;77(3):306-15.

61. Stiell IG, Brown SP, Christenson J, Cheskes S, Nichol G, Powell J, et al. What is the role of chest compression depth during out-of-hospital cardiac arrest resuscitation? Crit Care Med. 2012;40(4):1192-8. 
62. Vadeboncoeur T, Stolz U, Panchal A, Silver A, Venuti M, Tobin J, et al. Chest compression depth and survival in out-of-hospital cardiac arrest. Resuscitation. 2014;85(2):182-8.

63. Hellevuo H, Sainio M, Nevalainen R, Huhtala H, Olkkola KT, Tenhunen J, et al. Deeper chest compression - More complications for cardiac arrest patients? Resuscitation. $2013 ; 84(6): 760-5$.

64. Yannopoulos D, McKnite S, Aufderheide TP, Sigurdsson G, Pirrallo RG, Benditt D, et al. Effects of incomplete chest wall decompression during cardiopulmonary resuscitation on coronary and cerebral perfusion pressures in a porcine model of cardiac arrest. Resuscitation. 2005;64(3):363-72.

65. Glatz AC, Nishisaki A, Niles DE, Hanna BD, Eilevstjonn J, Diaz LK, et al. Sternal wall pressure comparable to leaning during CPR impacts intrathoracic pressure and haemodynamics in anaesthetized children during cardiac catheterization. Resuscitation. 2013;84(12):1674-9.

66. Jabre P, Penaloza A, Pinero D, Duchateau FX, Borron SW, Javaudin F, et al. Effect of BagMask Ventilation vs Endotracheal Intubation During Cardiopulmonary Resuscitation on Neurological Outcome After Out-of-Hospital Cardiorespiratory Arrest: A Randomized Clinical Trial. JAMA. 2018;319(8):779-87.

67. McMullan J, Gerecht R, Bonomo J, Robb R, McNally B, Donnelly J, et al. Airway management and out-of-hospital cardiac arrest outcome in the CARES registry. Resuscitation. 2014;85(5):617-22.

68. Shin SD, Ahn KO, Song KJ, Park CB, Lee EJ. Out-of-hospital airway management and cardiac arrest outcomes: A propensity score matched analysis. Resuscitation. 2012;83(3):313-9.

69. Nichol G, Leroux B, Wang H, Callaway CW, Sopko G, Weisfeldt M, et al. Trial of Continuous or Interrupted Chest Compressions during CPR. New England Journal of Medicine. 2015;373(23):2203-14.

70. Soar J, Nolan JP, Bottiger BW, Perkins GD, Lott C, Carli P, et al. European Resuscitation Council Guidelines for Resuscitation 2015: Section 3. Adult advanced life support. Resuscitation. 2015;95:100-47.

71. Link MS, Berkow LC, Kudenchuk PJ, Halperin HR, Hess EP, Moitra VK, et al. Part 7: Adult Advanced Cardiovascular Life Support: 2015 American Heart Association Guidelines Update for Cardiopulmonary Resuscitation and Emergency Cardiovascular Care. Circulation. 2015;132(18 Suppl 2):S444-64. 
72. Koster RW, Walker RG, Chapman FW. Recurrent ventricular fibrillation during advanced life support care of patients with prehospital cardiac arrest. Resuscitation. 2008;78(3):252-7.

73. Yaku H, Goto Y, Ohgoshi Y, Kawaguchi O, Oga K, Oka T, et al. Determinants of myocardial oxygen consumption in fibrillating dog hearts. Comparison between normothermia and hypothermia. J Thorac Cardiovasc Surg. 1993;105(4):679-88.

74. Sayen JJ, Sheldon WF, Peirce G, Kuo PT. Polarographic oxygen, the epicardial electrocardiogram and muscle contraction in experimental acute regional ischemia of the left ventricle. Circ Res. 1958;6(6):779-98.

75. Jennings RB, Reimer KA. Lethal myocardial ischemic injury. Am J Pathol. 1981;102(2):24155.

76. Braasch W, Gudbjarnason S, Puri PS, Ravens KG, Bing RJ. Early changes in energy metabolism in the myocardium following acute coronary artery occlusion in anesthetized dogs. Circ Res. 1968;23(3):429-38.

77. Choi HJ, Nguyen T, Park KS, Cha KC, Kim H, Lee KH, et al. Effect of cardiopulmonary resuscitation on restoration of myocardial ATP in prolonged ventricular fibrillation. Resuscitation. 2013;84(1):108-13.

78. Ditchey RV, Horwitz LD. Metabolic evidence of inadequate coronary blood flow during closed-chest resuscitation in dogs. Cardiovasc Res. 1985;19(7):419-25.

79. Weisfeldt ML, Becker LB. Resuscitation after cardiac arrest: a 3-phase time-sensitive model. JAMA. 2002;288(23):3035-8.

80. Weaver WD, Cobb LA, Dennis D, Ray R, Hallstrom AP, Copass MK. Amplitude of Ventricular-Fibrillation Waveform and Outcome after Cardiac-Arrest. Ann Intern Med. 1985;102(1):53-5.

81. Stewart AJ, Allen JD, Adgey AAJ. Frequency-Analysis of Ventricular-Fibrillation and Resuscitation Success. Q J Med. 1992;85(306):761-9.

82. Eftestol T, Sunde K, Aase SO, Husoy JH, Steen PA. Predicting outcome of defibrillation by spectral characterization and nonparametric classification of ventricular fibrillation in patients with out-of-hospital cardiac arrest. Circulation. 2000;102(13):1523-9.

83. Freese JP, Jorgenson DB, Liu PY, Innes J, Matallana L, Nammi K, et al. Waveform Analysis-Guided Treatment Versus a Standard Shock-First Protocol for the Treatment of Out-of-Hospital Cardiac Arrest Presenting in Ventricular Fibrillation: Results of an International Randomized, Controlled Trial. Circulation. 2013;128(9):995-1002. 
84. Ristagno G, Li Y, Fumagalli F, Finzi A, Quan W. Amplitude spectrum area to guide resuscitation-a retrospective analysis during out-of-hospital cardiopulmonary resuscitation in 609 patients with ventricular fibrillation cardiac arrest. Resuscitation. 2013;84(12):1697-703.

85. Carpenter J, Rea TD, Murray JA, Kudenchuk PJ, Eisenberg MS. Defibrillation waveform and post-shock rhythm in out-of-hospital ventricular fibrillation cardiac arrest. Resuscitation. 2003;59(2):189-96.

86. Freeman K, Hendey GW, Shalit M, Stroh G. Biphasic defibrillation does not improve outcomes compared to monophasic defibrillation in out-of-hospital cardiac arrest. Prehosp Emerg Care. 2008;12(2):152-6.

87. Gliner BE, White RD. Electrocardiographic evaluation of defibrillation shocks delivered to out-of-hospital sudden cardiac arrest patients. Resuscitation. 1999;41(2):133-44.

88. Hess EP, Atkinson EJ, White RD. Increased prevalence of sustained return of spontaneous circulation following transition to biphasic waveform defibrillation. Resuscitation. 2008;77(1):39-45.

89. Morrison LJ, Dorian P, Long J, Vermeulen M, Schwartz B, Sawadsky B, et al. Out-ofhospital cardiac arrest rectilinear biphasic to monophasic damped sine defibrillation waveforms with advanced life support intervention trial (ORBIT). Resuscitation. 2005;66(2):149-57.

90. van Alem AP, Chapman FW, Lank P, Hart AA, Koster RW. A prospective, randomised and blinded comparison of first shock success of monophasic and biphasic waveforms in out-ofhospital cardiac arrest. Resuscitation. 2003;58(1):17-24.

91. Schneider T, Martens PR, Paschen H, Kuisma M, Wolcke B, Gliner BE, et al. Multicenter, randomized, controlled trial of 150-J biphasic shocks compared with 200- to 360-J monophasic shocks in the resuscitation of out-of-hospital cardiac arrest victims. Optimized Response to Cardiac Arrest (ORCA) Investigators. Circulation. 2000;102(15):1780-7.

92. Kajino K, Iwami T, Berg RA, Hiraide A, Hayashi Y, Yukioka H, et al. Comparison of neurological outcomes following witnessed out-of-hospital ventricular fibrillation defibrillated with either biphasic or monophasic automated external defibrillators. Emerg Med J. 2009;26(7):492-6.

93. Killingsworth CR, Melnick SB, Chapman FW, Walker RG, Smith WM, Ideker RE, et al. Defibrillation threshold and cardiac responses using an external biphasic defibrillator with pediatric and adult adhesive patches in pediatric-sized piglets. Resuscitation. 2002;55(2):17785. 
94. Xie J, Weil MH, Sun S, Tang W, Sato Y, Jin X, et al. High-energy defibrillation increases the severity of postresuscitation myocardial dysfunction. Circulation. 1997;96(2):683-8.

95. Tang W, Weil MH, Sun S, Jorgenson D, Morgan C, Klouche K, et al. The effects of biphasic waveform design on post-resuscitation myocardial function. J Am Coll Cardiol. 2004;43(7):1228-35.

96. Stiell IG, Walker RG, Nesbitt LP, Chapman FW, Cousineau D, Christenson J, et al. BIPHASIC Trial: a randomized comparison of fixed lower versus escalating higher energy levels for defibrillation in out-of-hospital cardiac arrest. Circulation. 2007;115(12):1511-7.

97. Walcott GP, Melnick SB, Killingsworth CR, Ideker RE. Comparison of low-energy versus high-energy biphasic defibrillation shocks following prolonged ventricular fibrillation. Prehosp Emerg Care. 2010;14(1):62-70.

98. Berdowski J, Tijssen JGP, Koster RW. Chest Compressions Cause Recurrence of Ventricular Fibrillation After the First Successful Conversion by Defibrillation in Out-of-Hospital Cardiac Arrest. Circulation-Arrhythmia and Electrophysiology. 2010;3(1):72-8.

99. Jost D, Degrange H, Verret C, Hersan O, Banville IL, Chapman FW, et al. DEFI 2005: a randomized controlled trial of the effect of automated external defibrillator cardiopulmonary resuscitation protocol on outcome from out-of-hospital cardiac arrest. Circulation. 2010;121(14):1614-22.

100. Hess EP, Russell JK, Liu PY, White RD. A high peak current 150-J fixed-energy defibrillation protocol treats recurrent ventricular fibrillation (VF) as effectively as initial VF. Resuscitation. 2008;79(1):28-33.

101. Soar J, Callaway CW, Aibiki M, Bottiger BW, Brooks SC, Deakin CD, et al. Part 4: Advanced life support: 2015 International Consensus on Cardiopulmonary Resuscitation and Emergency Cardiovascular Care Science with Treatment Recommendations. Resuscitation. 2015;95:e71-120.

102. Sunde K, Jacobs I, Deakin CD, Hazinski MF, Kerber RE, Koster RW, et al. Part 6: Defibrillation: 2010 international consensus on cardiopulmonary resuscitation and emergency cardiovascular care science with treatment recommendations. Resuscitation. 2010;81 Suppl 1:e71-85.

103. 2005 International Consensus on Cardiopulmonary Resuscitation and Emergency Cardiovascular Care Science with Treatment Recommendations. Part 3: defibrillation. Resuscitation. 2005;67(2-3):203-11. 
104. White RD, Russell JK. Refibrillation, resuscitation and survival in out-of-hospital sudden cardiac arrest victims treated with biphasic automated external defibrillators. Resuscitation. 2002;55(1):17-23.

105. Eftestol T, Wik L, Sunde K, Steen PA. Effects of cardiopulmonary resuscitation on predictors of ventricular fibrillation defibrillation success during out-of-hospital cardiac arrest. Circulation. 2004;110(1):10-5.

106. Kern KB, Garewal HS, Sanders AB, Janas W, Nelson J, Sloan D, et al. Depletion of myocardial adenosine triphosphate during prolonged untreated ventricular fibrillation: effect on defibrillation success. Resuscitation. 1990;20(3):221-9.

107. Noc M, Weil MH, Gazmuri RJ, Sun S, Biscera J, Tang W. Ventricular fibrillation voltage as a monitor of the effectiveness of cardiopulmonary resuscitation. J Lab Clin Med. 1994;124(3):421-6.

108. Berg RA, Hilwig RW, Kern KB, Ewy GA. Precountershock cardiopulmonary resuscitation improves ventricular fibrillation median frequency and myocardial readiness for successful defibrillation from prolonged ventricular fibrillation: A randomized, controlled swine study. Annals of Emergency Medicine. 2002;40(6):563-70.

109. Kolarova J, Ayoub IM, Yi Z, Gazmuri RJ. Optimal timing for electrical defibrillation after prolonged untreated ventricular fibrillation. Crit Care Med. 2003;31(7):2022-8.

110. Sun S, Weng Y, Wu X, Tang K, Ye S, Chen W, et al. Optimizing the duration of CPR prior to defibrillation improves the outcome of CPR in a rat model of prolonged cardiac arrest. Resuscitation. 2011;82 Suppl 2:S3-7.

111. Cobb LA, Fahrenbruch CE, Walsh TR, Copass MK, Olsufka M, Breskin M, et al. Influence of cardiopulmonary resuscitation prior to defibrillation in patients with out-of-hospital ventricular fibrillation. JAMA-J Am Med Assoc. 1999;281(13):1182-8.

112. Wik L, Hansen TB, Fylling F, Steen T, Vaagenes P, Auestad BH, et al. Delaying defibrillation to give basic cardiopulmonary resuscitation to patients with out-of-hospital ventricular fibrillation: a randomized trial. JAMA. 2003;289(11):1389-95.

113. Baker PW, Conway J, Cotton C, Ashby DT, Smyth J, Woodman RJ, et al. Defibrillation or cardiopulmonary resuscitation first for patients with out-of-hospital cardiac arrests found by paramedics to be in ventricular fibrillation? A randomised control trial. Resuscitation. 2008;79(3):424-31.

114. Jacobs IG, Finn JC, Oxer HF, Jelinek GA. CPR before defibrillation in out-of-hospital cardiac arrest: a randomized trial. Emerg Med Australas. 2005;17(1):39-45. 
115. Ma MH-M, Chiang W-C, Ko PC-I, Yang C-W, Wang H-C, Chen S-Y, et al. A randomized trial of compression first or analyze first strategies in patients with out-of-hospital cardiac arrest: Results from an Asian community. Resuscitation.83(7):806-12.

116. Stiell IG, Nichol G, Leroux BG, Rea TD, Ornato JP, Powell J, et al. Early versus Later Rhythm Analysis in Patients with Out-of-Hospital Cardiac Arrest. New England Journal of Medicine. 2011;365(9):787-97.

117. Simpson PM, Goodger MS, Bendall JC. Delayed versus immediate defibrillation for out-ofhospital cardiac arrest due to ventricular fibrillation: A systematic review and meta-analysis of randomised controlled trials. Resuscitation. 2010;81(8):925-31.

118. Huang Y, He Q, Yang LJ, Liu GJ, Jones A. Cardiopulmonary resuscitation (CPR) plus delayed defibrillation versus immediate defibrillation for out-of-hospital cardiac arrest. The Cochrane database of systematic reviews. 2014;9:Cd009803.

119. Meier P, Baker P, Jost D, Jacobs I, Henzi B, Knapp G, et al. Chest compressions before defibrillation for out-of-hospital cardiac arrest: A meta-analysis of randomized controlled clinical trials. BMC Med. 2010;8(1):52.

120. Rea T, Prince D, Morrison L, Callaway C, Aufderheide T, Daya M, et al. Association between survival and early versus later rhythm analysis in out-of-hospital cardiac arrest: do agency-level factors influence outcomes? Ann Emerg Med. 2014;64(1):1-8.

121. Lexow K, Sunde K. Why Norwegian 2005 guidelines differs slightly from the ERC guidelines. Resuscitation. 2007;72(3):490-2.

122. Guidelines 2015, Adult advanced life support. Norwegian Resuscitation Council; 2016.

123. Edelson DP, Abella BS, Kramer-Johansen J, Wik L, Myklebust H, Barry AM, et al. Effects of compression depth and pre-shock pauses predict defibrillation failure during cardiac arrest. Resuscitation. 2006;71(2):137-45.

124. Yu T, Weil MH, Tang W, Sun S, Klouche K, Povoas H, et al. Adverse outcomes of interrupted precordial compression during automated defibrillation. Circulation. 2002;106(3):368-72.

125. Eftestol T, Sunde K, Steen PA. Effects of interrupting precordial compressions on the calculated probability of defibrillation success during out-of-hospital cardiac arrest. Circulation. 2002;105(19):2270-3.

126. Sell RE, Sarno R, Lawrence B, Castillo EM, Fisher R, Brainard C, et al. Minimizing pre- and post-defibrillation pauses increases the likelihood of return of spontaneous circulation (ROSC). Resuscitation. 2010;81(7):822-5. 
127. Vaillancourt C, Everson-Stewart S, Christenson J, Andrusiek D, Powell J, Nichol G, et al. The impact of increased chest compression fraction on return of spontaneous circulation for out-of-hospital cardiac arrest patients not in ventricular fibrillation. Resuscitation. 2011;82(12):1501-7.

128. Cheskes S, Schmicker RH, Christenson J, Salcido DD, Rea T, Powell J, et al. Perishock pause: an independent predictor of survival from out-of-hospital shockable cardiac arrest. Circulation. 2011;124(1):58-66.

129. Cheskes S, Schmicker RH, Verbeek PR, Salcido DD, Brown SP, Brooks S, et al. The impact of peri-shock pause on survival from out-of-hospital shockable cardiac arrest during the Resuscitation Outcomes Consortium PRIMED trial. Resuscitation. 2014;85(3):336-42.

130. Christenson J, Andrusiek D, Everson-Stewart S, Kudenchuk P, Hostler D, Powell J, et al. Chest Compression Fraction Determines Survival in Patients With Out-of-Hospital Ventricular Fibrillation. Circulation. 2009;120(13):1241-7.

131. Rea T, Olsufka M, Yin L, Maynard C, Cobb L. The relationship between chest compression fraction and outcome from ventricular fibrillation arrests in prolonged resuscitations. Resuscitation. 2014;85(7):879-84.

132. Brouwer TF, Walker RG, Chapman FW, Koster RW. Association Between Chest Compression Interruptions and Clinical Outcomes of Ventricular Fibrillation Out-of-Hospital Cardiac Arrest. Circulation. 2015;132(11):1030-7.

133. Aase SO, Eftestol T, Husoy JH, Sunde K, Steen PA. CPR artifact removal from human ECG using optimal multichannel filtering. IEEE Trans Biomed Eng. 2000;47(11):1440-9.

134. Ruiz J, Ayala U, Ruiz de Gauna S, Irusta U, Gonzalez-Otero D, Aramendi E, et al. Direct evaluation of the effect of filtering the chest compression artifacts on the uninterrupted cardiopulmonary resuscitation time. Am J Emerg Med. 2013;31(6):910-5.

135. Babaeizadeh S, Firoozabadi R, Han C, Helfenbein ED. Analyzing cardiac rhythm in the presence of chest compression artifact for automated shock advisory. J Electrocardiol. 2014;47(6):798-803.

136. Gong Y, Yu T, Chen B, He M, Li Y. Removal of cardiopulmonary resuscitation artifacts with an enhanced adaptive filtering method: an experimental trial. BioMed research international. 2014;2014:140438.

137. Aramendi E, Irusta U, Ayala U, Naas H, Kramer-Johansen J, Eftestol T. Filtering mechanical chest compression artefacts from out-of-hospital cardiac arrest data. Resuscitation. 2016;98:41-7. 
138. Fumagalli F, Silver AE, Tan Q, Zaidi N, Ristagno G. Cardiac rhythm analysis during ongoing cardiopulmonary resuscitation using the Analysis During Compressions with Fast Reconfirmation technology. Heart Rhythm. 2018;15(2):248-55.

139. Lloyd MS, Heeke B, Walter PF, Langberg JJ. Hands-on defibrillation: an analysis of electrical current flow through rescuers in direct contact with patients during biphasic external defibrillation. Circulation. 2008;117(19):2510-4.

140. Yu T, Ristagno G, Li Y, Bisera J, Weil MH, Tang W. The resuscitation blanket: a useful tool for "hands-on" defibrillation. Resuscitation. 2010;81(2):230-5.

141. Petley GW, Cotton AM, Deakin CD. Hands-on defibrillation: theoretical and practical aspects of patient and rescuer safety. Resuscitation. 2012;83(5):551-6.

142. Deakin CD, Lee-Shrewsbury V, Hogg K, Petley GW. Do clinical examination gloves provide adequate electrical insulation for safe hands-on defibrillation? I: Resistive properties of nitrile gloves. Resuscitation. 2013;84(7):895-9.

143. Kerber RE. Gloves to protect hands--and heart. Resuscitation. 2013;84(7):859-60.

144. McDonald JL. Systolic and mean arterial pressures during manual and mechanical CPR in humans. Ann Emerg Med. 1982;11(6):292-5.

145. Lilja GP, Hill M, Ruiz E, Clinton J. Clinical assessment of patients undergoing CPR in the emergency department. JACEP. 1979;8(2):81-3.

146. Niemann JT, Rosborough JP, Niskanen RA, Criley JM. Circulatory support during cardiac arrest using a pneumatic vest and abdominal binder with simultaneous high-pressure airway inflation. Ann Emerg Med. 1984;13(9 Pt 2):767-70.

147. Halperin HR, Guerci AD, Chandra N, Herskowitz A, Tsitlik JE, Niskanen RA, et al. Vest inflation without simultaneous ventilation during cardiac arrest in dogs: improved survival from prolonged cardiopulmonary resuscitation. Circulation. 1986;74(6):1407-15.

148. Criley JM, Blaufuss AH, Kissel GL. Cough-induced cardiac compression. Self-administered from of cardiopulmonary resuscitation. JAMA. 1976;236(11):1246-50.

149. Ward KR, Menegazzi JJ, Zelenak RR, Sullivan RJ, McSwain NE, Jr. A comparison of chest compressions between mechanical and manual CPR by monitoring end-tidal PCO2 during human cardiac arrest. Ann Emerg Med. 1993;22(4):669-74.

150. Halperin HR, Tsitlik JE, Gelfand M, Weisfeldt ML, Gruben KG, Levin HR, et al. A preliminary study of cardiopulmonary resuscitation by circumferential compression of the chest with use of a pneumatic vest. N Engl J Med. 1993;329(11):762-8. 
151. Zhang C, Wang Y, Liu H, Hao A, Xun J, Meng Q. [Comparative study for effects of barehanded CPR with Thumper cardiopulmonary resuscitator CPR in emergency department based on "the Utstein style"]. Zhonghua wei zhong bing ji jiu yi xue. 2017;29(10):937-9.

152. Lu XG, Kang X, Gong DB. [The clinical efficacy of Thumper modal 1007 cardiopulmonary resuscitation: a prospective randomized control trial]. Zhongguo Wei Zhong Bing Ji Jiu Yi Xue. 2010;22(8):496-7.

153. Dickinson ET, Verdile VP, Schneider RM, Salluzzo RF. Effectiveness of mechanical versus manual chest compressions in out-of-hospital cardiac arrest resuscitation: a pilot study. Am J Emerg Med. 1998;16(3):289-92.

154. Duchateau FX, Gueye P, Curac S, Tubach F, Broche C, Plaisance P, et al. Effect of the AutoPulse automated band chest compression device on hemodynamics in out-of-hospital cardiac arrest resuscitation. Intensive Care Med. 2010;36(7):1256-60.

155. Ikeno F, Kaneda H, Hongo Y, Sakanoue Y, Nolasco C, Emami S, et al. Augmentation of tissue perfusion by a novel compression device increases neurologically intact survival in a porcine model of prolonged cardiac arrest. Resuscitation. 2006;68(1):109-18.

156. Timerman S, Cardoso LF, Ramires JA, Halperin H. Improved hemodynamic performance with a novel chest compression device during treatment of in-hospital cardiac arrest. Resuscitation. 2004;61(3):273-80.

157. Steen S, Liao Q, Pierre L, Paskevicius A, Sjoberg T. Evaluation of LUCAS, a new device for automatic mechanical compression and active decompression resuscitation. Resuscitation. 2002;55(3):285-99.

158. Rubertsson S, Karlsten R. Increased cortical cerebral blood flow with LUCAS; a new device for mechanical chest compressions compared to standard external compressions during experimental cardiopulmonary resuscitation. Resuscitation. 2005;65(3):357-63.

159. Axelsson C, Karlsson T, Axelsson AB, Herlitz J. Mechanical active compressiondecompression cardiopulmonary resuscitation (ACD-CPR) versus manual CPR according to pressure of end tidal carbon dioxide $(\mathrm{P}(\mathrm{ET}) \mathrm{CO} 2)$ during $\mathrm{CPR}$ in out-of-hospital cardiac arrest (OHCA). Resuscitation. 2009;80(10):1099-103.

160. Wik L, Kiil S. Use of an automatic mechanical chest compression device (LUCAS) as a bridge to establishing cardiopulmonary bypass for a patient with hypothermic cardiac arrest. Resuscitation. 2005;66(3):391-4. 
161. Wagner H, Terkelsen CJ, Friberg H, Harnek J, Kern K, Lassen JF, et al. Cardiac arrest in the catheterisation laboratory: a 5-year experience of using mechanical chest compressions to facilitate PCI during prolonged resuscitation efforts. Resuscitation. 2010;81(4):383-7.

162. Spiro JR, White S, Quinn N, Gubran CJ, Ludman PF, Townend JN, et al. Automated cardiopulmonary resuscitation using a load-distributing band external cardiac support device for in-hospital cardiac arrest: A single centre experience of AutoPulse-CPR. Int J Cardiol. 2015;180:7-14.

163. Spiro J, Nadeem A, Doshi SN. Delayed left main stem obstruction following successful TAVI with an Edwards SAPIEN XT valve: successful resuscitation and percutaneous coronary intervention using a non-invasive automated chest compression device (AutoPulse). J Invasive Cardiol. 2012;24(5):224-8.

164. Krep H, Mamier M, Breil M, Heister U, Fischer M, Hoeft A. Out-of-hospital cardiopulmonary resuscitation with the AutoPulse ${ }^{\mathrm{TM}}$ system: A prospective observational study with a new load-distributing band chest compression device. Resuscitation. 2007;73(1):86-95.

165. Casner M, Andersen D, Isaacs SM. The impact of a new CPR assist device on rate of return of spontaneous circulation in out-of-hospital cardiac arrest. Prehospital Emergency Care. 2005;9(1):61-7.

166. Ong ME, Ornato JP, Edwards DP, Dhindsa HS, Best AM, Ines CS, et al. Use of an automated, load-distributing band chest compression device for out-of-hospital cardiac arrest resuscitation. JAMA. 2006;295(22):2629-37.

167. Hallstrom A, Rea TD, Sayre MR, Christenson J, Anton AR, Mosesso VN, Jr., et al. Manual chest compression vs use of an automated chest compression device during resuscitation following out-of-hospital cardiac arrest: a randomized trial. JAMA. 2006;295(22):2620-8.

168. Wik L, Olsen JA, Persse D, Sterz F, Lozano M, Jr., Brouwer MA, et al. Manual vs. integrated automatic load-distributing band CPR with equal survival after out of hospital cardiac arrest. The randomized CIRC trial. Resuscitation. 2014;85(6):741-8.

169. Koster RW, Beenen LF, van der Boom EB, Spijkerboer AM, Tepaske R, van der Wal AC, et al. Safety of mechanical chest compression devices AutoPulse and LUCAS in cardiac arrest: a randomized clinical trial for non-inferiority. Eur Heart J. 2017;38(40):3006-13.

170. Smekal D, Johansson J, Huzevka T, Rubertsson S. A pilot study of mechanical chest compressions with the LUCAS device in cardiopulmonary resuscitation. Resuscitation. 2011;82(6):702-6. 
171. Axelsson C, Nestin J, Svensson L, Axelsson AB, Herlitz J. Clinical consequences of the introduction of mechanical chest compression in the EMS system for treatment of out-ofhospital cardiac arrest-a pilot study. Resuscitation. 2006;71(1):47-55.

172. Rubertsson S, Lindgren E, Smekal D, Ostlund O, Silfverstolpe J, Lichtveld RA, et al. Mechanical chest compressions and simultaneous defibrillation vs conventional cardiopulmonary resuscitation in out-of-hospital cardiac arrest: the LINC randomized trial. JAMA. 2014;311(1):53-61.

173. Perkins GD, Lall R, Quinn T, Deakin CD, Cooke MW, Horton J, et al. Mechanical versus manual chest compression for out-of-hospital cardiac arrest (PARAMEDIC): a pragmatic, cluster randomised controlled trial. Lancet. 2014;385(9972):947-55.

174. Govindarajan P, Lin L, Landman A, McMullan JT, McNally BF, Crouch AJ, et al. Practice variability among the EMS systems participating in Cardiac Arrest Registry to Enhance Survival (CARES). Resuscitation. 2012;83(1):76-80.

175. Olsen JA, Brunborg C, Steinberg M, Persse D, Sterz F, Lozano M, Jr., et al. Pre-shock chest compression pause effects on termination of ventricular fibrillation/tachycardia and return of organized rhythm within mechanical and manual cardiopulmonary resuscitation. Resuscitation. 2015;93:158-63.

176. Li Y, Wang H, Cho JH, Quan W, Freeman G, Bisera J, et al. Defibrillation delivered during the upstroke phase of manual chest compression improves shock success. Crit Care Med. 2010;38(3):910-5.

177. Li Y, Yu T, Ristagno G, Chung SP, Bisera J, Quan W, et al. The optimal phasic relationship between synchronized shock and mechanical chest compressions. Resuscitation. 2010;81(6):724-9.

178. Lurie KG, Lindo C, Chin J. CPR: the P stands for plumber's helper. JAMA. 1990;264(13):1661.

179. Cohen TJ, Tucker KJ, Lurie KG, Redberg RF, Dutton JP, Dwyer KA, et al. Active compression-decompression. A new method of cardiopulmonary resuscitation. Cardiopulmonary Resuscitation Working Group. JAMA. 1992;267(21):2916-23.

180. Cohen TJ, Tucker KJ, Redberg RF, Lurie KG, Chin MC, Dutton JP, et al. Active compression-decompression resuscitation: a novel method of cardiopulmonary resuscitation. Am Heart J. 1992;124(5):1145-50. 
181. Lindner KH, Pfenninger EG, Lurie KG, Schurmann W, Lindner IM, Ahnefeld FW. Effects of active compression-decompression resuscitation on myocardial and cerebral blood flow in pigs. Circulation. 1993;88(3):1254-63.

182. Tucker KJ, Khan JH, Savitt MA. Active compression-decompression resuscitation: effects on pulmonary ventilation. Resuscitation. 1993;26(2):125-31.

183. Chang MW, Coffeen P, Lurie KG, Shultz J, Bache RJ, White CW. Active compressiondecompression CPR improves vital organ perfusion in a dog model of ventricular fibrillation. Chest. 1994;106(4):1250-9.

184. Wik L, Naess PA, Ilebekk A, Steen PA. Simultaneous active compression-decompression and abdominal binding increase carotid blood flow additively during cardiopulmonary resuscitation (CPR) in pigs. Resuscitation. 1994;28(1):55-64.

185. Wik L, Naess PA, Ilebekk A, Nicolaysen G, Steen PA. Effects of various degrees of compression and active decompression on haemodynamics, end-tidal $\mathrm{CO} 2$, and ventilation during cardiopulmonary resuscitation of pigs. Resuscitation. 1996;31(1):45-57.

186. Tucker KJ, Redberg RF, Schiller NB, Cohen TJ. Active compression-decompression resuscitation: analysis of transmitral flow and left ventricular volume by transesophageal echocardiography in humans. Cardiopulmonary Resuscitation Working Group. J Am Coll Cardiol. 1993;22(5):1485-93.

187. Shultz JJ, Coffeen P, Sweeney M, Detloff B, Kehler C, Pineda E, et al. Evaluation of standard and active compression-decompression CPR in an acute human model of ventricular fibrillation. Circulation. 1994;89(2):684-93.

188. Guly UM, Robertson CE. Active decompression improves the haemodynamic state during cardiopulmonary resuscitation. Br Heart J. 1995;73(4):372-6.

189. Orliaguet GA, Carli PA, Rozenberg A, Janniere D, Sauval P, Delpech P. End-tidal carbon dioxide during out-of-hospital cardiac arrest resuscitation: comparison of active compressiondecompression and standard CPR. Ann Emerg Med. 1995;25(1):48-51.

190. Malzer R, Zeiner A, Binder M, Domanovits H, Knappitsch G, Sterz F, et al. Hemodynamic effects of active compression-decompression after prolonged CPR. Resuscitation. 1996;31(3):243-53.

191. Mauer D, Schneider T, Elich D, Dick W. Carbon dioxide levels during pre-hospital active compression--decompression versus standard cardiopulmonary resuscitation. Resuscitation. 1998;39(1-2):67-74. 
192. Plaisance P, Lurie KG, Vicaut E, Adnet F, Petit JL, Epain D, et al. A comparison of standard cardiopulmonary resuscitation and active compression-decompression resuscitation for outof-hospital cardiac arrest. French Active Compression-Decompression Cardiopulmonary Resuscitation Study Group. N Engl J Med. 1999;341(8):569-75.

193. Lurie KG, Shultz JJ, Callaham ML, Schwab TM, Gisch T, Rector T, et al. Evaluation of active compression-decompression CPR in victims of out-of-hospital cardiac arrest. JAMA. 1994;271(18):1405-11.

194. Tucker KJ, Galli F, Savitt MA, Kahsai D, Bresnahan L, Redberg RF. Active compressiondecompression resuscitation: effect on resuscitation success after in-hospital cardiac arrest. J Am Coll Cardiol. 1994;24(1):201-9.

195. Luiz T, Ellinger K, Denz C. Active compression-decompression cardiopulmonary resuscitation does not improve survival in patients with prehospital cardiac arrest in a physician-manned emergency medical system. J Cardiothorac Vasc Anesth. 1996;10(2):17886.

196. Mauer D, Schneider T, Dick W, Withelm A, Elich D, Mauer M. Active compressiondecompression resuscitation: a prospective, randomized study in a two-tiered EMS system with physicians in the field. Resuscitation. 1996;33(2):125-34.

197. Schwab TM, Callaham ML, Madsen CD, Utecht TA. A randomized clinical trial of active compression-decompression CPR vs standard CPR in out-of-hospital cardiac arrest in two cities. JAMA. 1995;273(16):1261-8.

198. Stiell IG, Hébert PC, Wells GA, et al. The ontario trial of active compression-decompression cardiopulmonary resuscitation for in-hospital and prehospital cardiac arrest. JAMA. 1996;275(18):1417-23.

199. Nolan J, Smith G, Evans R, McCusker K, Lubas P, Parr M, et al. The United Kingdom prehospital study of active compression-decompression resuscitation. Resuscitation. 1998;37(2):119-25.

200. Skogvoll E, Wik L. Active compression-decompression cardiopulmonary resuscitation: a population-based, prospective randomised clinical trial in out-of-hospital cardiac arrest. Resuscitation. 1999;42(3):163-72.

201. Aufderheide TP, Pirrallo RG, Provo TA, Lurie KG. Clinical evaluation of an inspiratory impedance threshold device during standard cardiopulmonary resuscitation in patients with out-of-hospital cardiac arrest. Crit Care Med. 2005;33(4):734-40. 
202. Wolcke BB, Mauer DK, Schoefmann MF, Teichmann H, Provo TA, Lindner KH, et al. Comparison of standard cardiopulmonary resuscitation versus the combination of active compression-decompression cardiopulmonary resuscitation and an inspiratory impedance threshold device for out-of-hospital cardiac arrest. Circulation. 2003;108(18):2201-5.

203. Aufderheide TP, Frascone RJ, Wayne MA, Mahoney BD, Swor RA, Domeier RM, et al. Comparative Effectiveness of Standard CPR versus Active Compression Decompression CPR with Augmentation of Negative Intrathoracic Pressure for Treatment of Out-of-Hospital Cardiac Arrest: Results from a Randomized Prospective Study. Lancet. 2011;377(9762):30111.

204. Frascone RJ, Wayne MA, Swor RA, Mahoney BD, Domeier RM, Olinger ML, et al. Treatment of non-traumatic out-of-hospital cardiac arrest with active compression decompression cardiopulmonary resuscitation plus an impedance threshold device. Resuscitation. 2013;84(9):1214-22.

205. Sunde K, Wik L, Steen PA. Quality of mechanical, manual standard and active compressiondecompression CPR on the arrest site and during transport in a manikin model. Resuscitation. 1997;34(3):235-42.

206. Skogvoll E, Wik L. Active compression-decompression cardiopulmonary resuscitation (ACD-CPR) compared with standard CPR in a manikin model--decompression force, compression rate, depth and duration. Resuscitation. 1997;34(1):11-6.

207. Shultz JJ, Mianulli MJ, Gisch TM, Coffeen PR, Haidet GC, Lurie KG. Comparison of exertion required to perform standard and active compression-decompression cardiopulmonary resuscitation. Resuscitation. 1995;29(1):23-31.

208. Electrical impedance. Encyclopaedia Britannica. Accessed September 26, 2017.

209. Kubicek WG, Karnegis JN, Gatterso.Rp, Witsoe DA, Mattson RH. Development and Evaluation of an Impedance Cardiac Output System. Aerosp Med. 1966;37(12):1208-\&.

210. Milsom I, Sivertsson R, Biber B, Olsson T. Measurement of stroke volume with impedance cardiography. Clin Physiol. 1982;2(5):409-17.

211. Pomerantz M, Delgado F, Eiseman B. Clinical evaluation of transthoracic electrical impedance as a guide to intrathoracic fluid volumes. Ann Surg. 1970;171(5):686-94.

212. Smith SA, Russell AE, West MJ, Chalmers J. Automated non-invasive measurement of cardiac output: comparison of electrical bioimpedance and carbon dioxide rebreathing techniques. Br Heart J. 1988;59(3):292-8. 
213. Losert H, Risdal M, Sterz F, Nysaether J, Koehler K, Eftestol T, et al. Thoracic impedance changes measured via defibrillator pads can monitor ventilation in critically ill patients and during cardiopulmonary resuscitation. Crit Care Med. 2006;34(9):2399-405.

214. Stecher FS, Olsen JA, Stickney RE, Wik L. Transthoracic impedance used to evaluate performance of cardiopulmonary resuscitation during out of hospital cardiac arrest. Resuscitation. 2008;79(3):432-7.

215. Lerner EB, Persse D, Souders CM, Sterz F, Malzer R, Lozano M, Jr., et al. Design of the Circulation Improving Resuscitation Care (CIRC) Trial: a new state of the art design for outof-hospital cardiac arrest research. Resuscitation. 2011;82(3):294-9.

216. Von Hippel PT. Regression with missing ys: an improved strategy for analyzing multiply imputed data. Sociological Methodology. 2007;37(1):83-117.

217. Lown B, Amarasin.R, Neuman J. New Method for Terminating Cardiac Arrhythmias - Use of Synchronized Capacitor Discharge. JAMA-J Am Med Assoc. 1962;182(5):548-\&.

218. Mazer CD, Greene MB, Misale PS, Newman D, Dorian P. Transcutaneous T wave shock: a universal method for ventricular fibrillation induction. Pacing Clin Electrophysiol. 1997;20(12 Pt 1):2930-5.

219. Ornato JP, Shipley J, Powell RG, Racht EM. Inappropriate Electrical Countershocks by an Automated External Defibrillator. Annals of Emergency Medicine. 1992;21(10):1278-81.

220. Kramer-Johansen J, Edelson DP, Abella BS, Becker LB, Wik L, Steen PA. Pauses in chest compression and inappropriate shocks: a comparison of manual and semi-automatic defibrillation attempts. Resuscitation. 2007;73(2):212-20.

221. Sanders AB. Capnometry in emergency medicine. Ann Emerg Med. 1989;18(12):1287-90.

222. Hollinger CB HJ. Capnography and respiratory monitoring. Textbook of Critical Care, 3rd Edition, WB Saunders Company. 1995;Chapter 36:pp 305-11.

223. Trillo G, von Planta M, Kette F. ETCO2 monitoring during low flow states: clinical aims and limits. Resuscitation. 1994;27(1):1-8.

224. Kalenda Z. The capnogram as a guide to the efficacy of cardiac massage. Resuscitation. 1978;6(4):259-63.

225. Heradstveit BE, Sunde K, Sunde GA, Wentzel-Larsen T, Heltne JK. Factors complicating interpretation of capnography during advanced life support in cardiac arrest--a clinical retrospective study in 575 patients. Resuscitation. 2012;83(7):813-8. 
226. Pellis T, Bisera J, Tang W, Weil MH. Expanding automatic external defibrillators to include automated detection of cardiac, respiratory, and cardiorespiratory arrest. Crit Care Med. 2002;30(4 Suppl):S176-8.

227. Wik L, Kramer-Johansen J, Myklebust H, Sorebo H, Svensson L, Fellows B, et al. Quality of cardiopulmonary resuscitation during out-of-hospital cardiac arrest. JAMA. 2005;293(3):299-304.

228. Alonso E, Aramendi E, Daya M, Irusta U, Chicote B, Russell JK, et al. Circulation detection using the electrocardiogram and the thoracic impedance acquired by defibrillation pads. Resuscitation. 2016;99:56-62.

229. Dubner SJ, Gimeno GM, Elencwajg B, Leguizamon J, Tronge JE, Quinteiro R. Ventricular fibrillation with spontaneous reversion on ambulatory ECG in the absence of heart disease. Am Heart J. 1983;105(4):691-3.

230. Lown B, Temte JV, Reich P, Gaughan C, Regestein Q, Hal H. Basis for recurring ventricular fibrillation in the absence of coronary heart disease and its management. N Engl J Med. 1976;294(12):623-9.

231. Choquette G, Wasserman F, Lisker S, Bellet S. Spontaneous reversion of ventricular fibrillation to normal sinus rhythm in a case of acute myocardial infarction. Am Heart J. 1956;51(3):455-9.

232. Priest WM. Ventricular fibrillation recorded 10 hours before death from myocardial infarction. Lancet. 1949;2(6581):699.

233. Sunde K, Eftestol T, Askenberg C, Steen PA. Quality assessment of defribrillation and advanced life support using data from the medical control module of the defibrillator. Resuscitation. 1999;41(3):237-47.

234. Robertson C, Steen P, Adgey J, Bossaert L, Carli P, Chamberlain D, et al. The 1998 European Resuscitation Council guidelines for adult advanced life support: A statement from the Working Group on Advanced Life Support, and approved by the executive committee. Resuscitation. 1998;37(2):81-90.

235. Osorio J, Dosdall DJ, Robichaux RP, Jr., Tabereaux PB, Ideker RE. In a swine model, chest compressions cause ventricular capture and, by means of a long-short sequence, ventricular fibrillation. Circulation Arrhythmia and electrophysiology. 2008;1(4):282-9.

236. Osorio J, Dosdall DJ, Tabereaux PB, Robichaux RP, Jr., Stephens S, Kerby JD, et al. Effect of chest compressions on ventricular activation. Am J Cardiol. 2012;109(5):670-4. 
237. Pell AC, Guly UM, Sutherland GR, Steedman DJ, Bloomfield P, Robertson C. Mechanism of closed chest cardiopulmonary resuscitation investigated by transoesophageal echocardiography. J Accid Emerg Med. 1994;11(3):139-43.

238. Zipes DP, Fischer J, King RM, Nicoll Ad, Jolly WW. Termination of ventricular fibrillation in dogs by depolarizing a critical amount of myocardium. Am J Cardiol. 1975;36(1):37-44.

239. Chen PS, Shibata N, Dixon EG, Martin RO, Ideker RE. Comparison of the defibrillation threshold and the upper limit of ventricular vulnerability. Circulation. 1986;73(5):1022-8.

240. Lerman BB, Deale OC. Relation between transcardiac and transthoracic current during defibrillation in humans. Circ Res. 1990;67(6):1420-6.

241. Perneger TV. What's wrong with Bonferroni adjustments. BMJ. 1998;316(7139):1236-8.

242. Rothman KJ. No adjustments are needed for multiple comparisons. Epidemiology. 1990;1(1):43-6.

243. Otlewski MP, Geddes LA, Pargett M, Babbs CF. Methods for calculating coronary perfusion pressure during CPR. Cardiovasc Eng. 2009;9(3):98-103.

244. Halperin HR, Tsitlik JE, Guerci AD, Mellits ED, Levin HR, Shi AY, et al. Determinants of blood flow to vital organs during cardiopulmonary resuscitation in dogs. Circulation. 1986;73(3):539-50.

245. Sunde K, Wik L, Naess PA, Ilebekk A, Nicolaysen G, Steen PA. Effect of different compression--decompression cycles on haemodynamics during ACD-CPR in pigs. Resuscitation. 1998;36(2):123-31.

246. Mosher P, Ross J, Jr., McFate PA, Shaw RF. Control of Coronary Blood Flow by an Autoregulatory Mechanism. Circ Res. 1964;14:250-9.

247. Ditchey RV, Winkler JV, Rhodes CA. Relative lack of coronary blood flow during closedchest resuscitation in dogs. Circulation. 1982;66(2):297-302.

248. Langhelle A, Stromme T, Sunde K, Wik L, Nicolaysen G, Steen PA. Inspiratory impedance threshold valve during CPR. Resuscitation. 2002;52(1):39-48.

249. R N. Lehrbuch der Anatomie der Haustiere Band III: Kreislaufsystem, Haut und Hautorgane: Verlag Paul Parey; 1976.

250. Baubin M, Sumann G, Rabl W, Eibl G, Wenzel V, Mair P. Increased frequency of thorax injuries with ACD-CPR. Resuscitation. 1999;41(1):33-8. 
251. Rabl W, Baubin M, Broinger G, Scheithauer R. Serious complications from active compression-decompression cardiopulmonary resuscitation. Int J Legal Med. 1996;109(2):84-9.

252. Hoke RS, Chamberlain D. Skeletal chest injuries secondary to cardiopulmonary resuscitation. Resuscitation. 2004;63(3):327-38. 

Reprints of Papers I-III 

I 

Clinical paper

\title{
Minimizing pre-shock chest compression pauses in a cardiopulmonary resuscitation cycle by performing an earlier rhythm analysis ${ }^{\text {in }}$
}

\author{
Mikkel T. Steinberg ${ }^{\mathrm{a}, \mathrm{b}, *}$, Jan-Aage Olsen ${ }^{\mathrm{b}, \mathrm{c}}$, Cathrine Brunborg ${ }^{\mathrm{d}}$, David Persse ${ }^{\mathrm{e}}$, \\ Pierre M. van Grunsven ${ }^{1}$, David T. Travis ${ }^{g}$, E. Brooke Lerner ${ }^{m}$, Lars Wik ${ }^{b}$ \\ a Medical Student Research Program, University of Oslo, Oslo, Norway \\ ${ }^{\mathrm{b}}$ Norwegian National Advisory Unit on Prehospital Emergency Medicine, Oslo University Hospital, Oslo, Norway \\ ${ }^{c}$ Institute of Clinical Medicine, University of Oslo, Oslo, Norway \\ ${ }^{\mathrm{d}}$ Department of Statistics and Epidemiology, Oslo University Hospital, Oslo, Norway \\ e Houston Fire Department and the Baylor College of Medicine, Houston, TX, United States \\ ${ }^{\mathrm{f}}$ Department of Emergency Medicine, Medical University of Vienna, Vienna, Austria \\ ${ }^{\mathrm{g}}$ Hillsborough County Fire Rescue, Tampa, FL, United States \\ h Department of Emergency Medicine, Lake Erie College, Bradenton, FL, United States \\ ${ }^{i}$ Heart Lung Center, Department of Cardiology, Radboud University Nijmegen Medical Center, Nijmegen, The Netherlands \\ j Gold Cross Ambulance Service, Appleton Neenah-Menasha and Grand Chute Fire Departments, Neenah, WI, United States \\ ${ }^{\mathrm{k}}$ Theda Clark Regional Medical Center, Neenah, WI, United States \\ ${ }^{1}$ Regional Ambulance Service Gelderland-Zuid, Nijmegen, The Netherlands \\ ${ }^{\mathrm{m}}$ Department of Emergency Medicine, Medical College of Wisconsin, Milwaukee, WI, United States
} Fritz Sterz ${ }^{\mathrm{f}}$, Michael Lozano Jr ${ }^{\mathrm{g}, \mathrm{h}}$, Marc A. Brouwer ${ }^{\mathrm{i}}$, Mark Westfall ${ }^{\mathrm{j}, \mathrm{k}}$, Chris M. Souders ${ }^{\mathrm{e}}$,

\section{A R T I C L E I N F O}

\section{Article history:}

Received 22 June 2014

Received in revised form 3 October 2014

Accepted 15 November 2014

\section{Keywords:}

Cardiopulmonary resuscitation

Defibrillation

Ventricular fibrillation

Guidelines

\begin{abstract}
A B S T R A C T
Background: Guidelines recommend 2 min of CPR after defibrillation attempts followed by ECG analysis during chest compression pause. This pause may reduce the likelihood of return of spontaneous circulation (ROSC) and survival. We have evaluated the possibility of analysing the rhythm earlier in the CPR cycle in an attempt to replace immediate pre-shock rhythm analysis.

Methods and results: The randomized Circulation Improving Resuscitation Care (CIRC) trial included patients with out of hospital cardiac arrest of presumed cardiac aetiology. Defibrillator data were used to categorize ECG rhythms as shockable or non-shockable 1 min post-shock and immediately before next shock. ROSC was determined from end-tidal $\mathrm{CO}_{2}$, transthoracic impedance (TTI), and patient records. TTI was used to identify chest compressions. Artefact free ECGs were categorized during periods without chest compressions. Episodes without ECG or TTI data or with undeterminable ECG rhythm were excluded. Data were analyzed using descriptive statistics.

Of 1657 patients who received 3409 analysable shocks, the rhythm was shockable in 1529 (44.9\%) cases 1 min post-shock, 13 (0.9\%) of which were no longer shockable immediately prior to next possible shock. Of these, three had converted to asystole, seven to PEA and three to ROSC.

Conclusion: While a shockable rhythm 1 min post-shock was present also immediately before next possible defibrillation attempt in most cases, three patients had ROSC. Studies are needed to document if moving the pre-shock rhythm analysis will increase shocks delivered to organized rhythms, and if it will increase shock success and survival.
\end{abstract}

(C) 2014 Elsevier Ireland Ltd. All rights reserved.

\footnotetext{
is A Spanish translated version of the abstract of this article appears as Appendix in the final online version at http://dx.doi.org/10.1016/j.resuscitation.2014.11.012. * Corresponding author at: Steinberg, Oslo University Hospital-Ullevål, Norwegian National Advisory Unit on Prehospital Emergency Medicine (NAKOS), Building 31, PO Box 4956 Nydalen, NO-0424 Oslo, Norway.

E-mail address: mikkelts@studmed.uio.no (M.T. Steinberg).
} 


\section{Introduction}

In patients with cardiac arrest European Resuscitation Council (ERC) 2010 guidelines recommend two-minute cycles of cardiopulmonary resuscitation (CPR) followed by ECG rhythm analysis immediately prior to possible defibrillation attempts. ${ }^{1,2}$ This requires a pause in chest compressions to avoid corrupting the ECG signals by compression artefacts. Studies indicate that pauses immediately before defibrillation attempts may affect termination of fibrillation, return of spontaneous circulation (ROSC) and survival. ${ }^{3-8}$ Chest compression fraction (time with compressions as fraction of total CPR time) has also been reported to affect survival, ${ }^{9,10}$ but compression pauses immediately pre-shock have been reported to affect survival independent of the compression fraction. ${ }^{7}$ Guidelines therefore emphasize minimizing immediate pre-shock compression pauses as such, not only reducing pauses in chest compressions in general. ${ }^{1,2}$ Pre-shock pauses can be reduced to a few seconds if the defibrillator software allows chest compressions during defibrillator charging. ${ }^{2}$ An additional approach independent of the type of defibrillator would be if the pause for ECG analysis could be moved earlier in the CPR cycle. This approach would require that the analysis has sufficient predictive accuracy for the rhythm at the time of the next possible defibrillation attempt.

The Circulation Improving Resuscitation Care (CIRC) trial compared manual and mechanical CPR in out-of-hospital cardiac arrest (OHCA) patients in US and Europe, and has a large database with continuous ECG recordings. ${ }^{11} 2005$ ERC and AHA guidelines were followed with the exemption of a 3 min CPR cycle with rhythm analysis 1 min after defibrillation attempts according to Norwegian CPR guidelines. ${ }^{1,2,12}$ If the rhythm is shockable at this point, chest compressions are continued for another 2 min before a second rhythm analysis is carried out immediately before a possible defibrillation attempt. ${ }^{12}$ The CIRC study therefore gives the unique opportunity to evaluate an artefact free ECG at two points during a CPR cycle and thereby assess the stability of a shockable rhythm during chest compressions.

We hypothesize that a shockable rhythm 1 min post-shock stays shockable after 2 min of CPR, until next defibrillation attempt. This knowledge may be useful for CPR guideline processes, both for twoand three-minute CPR cycles.

\section{Methods}

The multi-centre CIRC trial was carried out in Houston, TX, Fox Valley, WI and Hillsborough County, FL in the US, Vienna in Austria and Nijmegen in the Netherlands. The aim was to compare survival to hospital discharge for OHCA patients randomized to receive either high quality manual chest compressions (Mcc) or integrated (patient receives manual compressions while the mechanical device is deployed) automatic mechanical chest compressions delivered by a Load Distributing Band (LDB-Cc) (AutoPuls®, ZOLL Medical, Chelmsford, MA). The CIRC trial was conducted under exception from informed consent for emergency research, and it was approved by the Institutional Review Board (IRB) (US sites) or ethics committee (European sites) for each lead EMS agency and each hospital likely to receive patients. The study protocol is described in detail in a previous methods paper. ${ }^{13}$

Continuous ECG, transthoracic impedance (TTI), defibrillation attempt(s), end-tidal $\mathrm{CO}_{2}\left(\mathrm{ETCO}_{2}\right)$, and time intervals were continuously recorded and stored by the defibrillators which were used in both manual and semiautomatic mode. Both LIFEPAK® 12, 15 and 500 (Physio-Control, Redmond, WA, US) and Zoll E Series ${ }^{\circledR}$ and AED Pro ${ }^{\circledR}$ (ZOLL Medical, Chelmsford, MA, US) were used in the trial. These defibrillators do not have filtering technology with the ability to remove real-time ECG artefacts. The data were then downloaded to CIRC central server after each resuscitation attempt. ${ }^{13}$ Electronic defibrillator data case files were analyzed using CodeStat 8.0 Data Review Software (Physio-Control, Redmond, WA, US) or RescueNet ${ }^{\circledR}$ Code Review 5.5.3 (ZOLL Medical Corporation, Chelmsford, MA, USA). Software TTI filtering in CodeStat 8.0 allowed us to filter the TTI signal, and thereby more accurately distinguish between the different TTI curves of the compressions and the ventilations. This enabled us to more accurately score the cases.

The use of TTI is a documented method of evaluating performance of CPR and alignment to guidelines or a study protocol. ${ }^{14}$ Compressions and ventilations can be determined from TTI waveform analysis. ${ }^{14}$ Chest compressions can generate ECG artefacts that may look like VF/VT. ECG evaluation is therefore much more accurate if a parallel TTI graph indicates if chest compressions are given or not. According to CIRC protocol EMS personnel were to stop chest compressions ( $\max 10 \mathrm{~s}$ ) and determine rhythm by feeling for pulses and evaluate the defibrillator monitor ECG. We analyzed ECG rhythms retrospectively during compression pauses (verified with a flat TTI graph) of minimum $2 \mathrm{~s}$ to avoid compression artefacts and interference on the ECG. Only artefact free ECG data was scored. All rhythms were scored by one analyst. Uncertain cases were scored after a discussion with expert analysts, both anaesthesiologist and cardiologist.

For all cardiac arrest episodes we determined initial rhythm as ventricular fibrillation (VF), ventricular tachycardia (VT), asystole, pulseless electric activity (PEA, defined as electrical activity with QRS-waves of any width at an average rate of $10>$ beats per minute) or ROSC (determined using ECG, TTI, ETCO 2 and notions in the case report forms (based on ambulance records)). Shocks without compression pause for rhythm analysis or with missing ECG or impedance signal at either $1 \mathrm{~min}$ and/or $3 \mathrm{~min}$ post-shock were excluded. The presence of ICD shocks in-between rhythm analyses or lack of CPR also led to exclusion.

For each defibrillation attempt we determined the rhythm pre-shock, $1 \mathrm{~min}$ and $3 \mathrm{~min}$ post-shock (immediately before next possible defibrillation attempt). It is difficult for EMS personnel in the field to adhere exactly to guidelines regarding the timing of rhythm analysis. Therefore, the one- and three-minute rhythm analyses were accepted and included for analysis when carried out between 30 and $90 \mathrm{~s}$ and between 120 and 240 s post-shock, respectively.

Shocks delivered to a non-shockable rhythm (asystole or an organized rhythm defined as ROSC or PEA) were categorized as inappropriate. We examined the results of inappropriate shocks by determining the rhythm $5 \mathrm{~s}$ post-shock.

During resuscitation patients could receive adrenaline, amiodarone, atropine, vasopressin and/or lidocaine depending on local emergency medical service (EMS) drug guidelines. If drugs were administrated it was noted in the ambulance journal, but the timing of drug delivery was not documented.

Descriptive analyses were performed using SPSS version 21.0 (IBM SPSS Inc., Chicago, IL, USA), and presented as proportions or medians with Inter Quartile Ranges (IQR) and 25\% and 75\% percentiles. Multiple imputations were not considered because shocks are the dependent variable with repeated measurements. Since only descriptive analysis was performed imputed dependent variable contributes no information to the analysis. ${ }^{15}$

\section{Results}

Of 4231 CIRC patients, 1657 (39.4\%) received one or more shocks (totally 5336 shocks). Forty-three patients were excluded due to defibrillator data import failure, missing ECG or TTI data, or undeterminable initial rhythm. The 1614 included patients received 
Table 1

Time intervals for initial shockable and non-shockable rhythms presented as median minutes:seconds with IQR.

\begin{tabular}{lcr}
\hline & Initial shockable rhythm $\left(n=893^{*}\right)$ & Initial non-shockable rhythm $\left(n=718^{*}\right)$ \\
\hline 911 Call to EMS arrival at scene & $6: 55(5: 23,8: 41)$ & $6: 49(5: 18,8: 48)$ \\
911 Call to first shock & $13: 04(10: 39,16: 20)$ & $27: 46(20: 44,38: 09)$ \\
EMS on scene to pads on & $3: 22(2: 12,5: 16)$ & $4: 05(2: 47,6: 02)$ \\
Pads on to shockable rhythm & & $13: 46(7: 53,23: 12)$ \\
Pads on to first shock & $2: 27(00: 50,03: 53)$ & $15: 25(9: 16,24: 43)$ \\
\hline
\end{tabular}

" Missing time intervals for 3 patients.

4867 shocks, of which 1458 (30\%) were excluded due to lack of criteria for adequate post-shock analysis, leaving 3409 shocks for analysis. For the patients who received one shock or more, the initial rhythm was shockable (VF/VT) in 894 patients (55.4\%) and non-shockable (PEA/asystole) in 720 patients (44.6\%). The time intervals "911 Call to EMS arrival at scene" and "EMS on scene to pads on" are presented in Table 1.

Of the 3409 shocks for post-shock analysis, 1880 rhythms were non-shockable 1 min post-shock, of which 342 (18.2\%) converted to shockable 3 min post-shock. Of the 1529 shockable rhythms $1 \mathrm{~min}$ post-shock, 1516 (99.1\%) (after M-cc: 99.4\%, after LDB-cc: 98.8\%) were still shockable 3 min post-shock (immediately before next possible defibrillation attempt). Thirteen $(0.9 \%)$ shockable rhythms $1 \mathrm{~min}$ post-shock had converted to a non-shockable rhythm $3 \mathrm{~min}$ post-shock during CPR and not in association with defibrillation. Of these, three converted to asystole (two after M-cc, one after LDBcc), seven to PEA (three after M-cc and four after LDB-cc) and three to ROSC (one after M-cc and two after LDB-cc) (Fig. 1).

In the CIRC trial, $370(6.9 \%)$ of 5336 shocks were classified as inappropriate with 148 (40\%) delivered to organized rhythms and $222(60 \%)$ to asystole. Of the inappropriate shocks 160 had an analysable rhythm without compression artefacts both preshock and $5 \mathrm{~s}$ post-shock. The rhythm did not change post-shock in 154/160 (96.3\%) of these instances. Sixty-six of the 160 inappropriate shocks were given to organized rhythms, all were identified as PEA and no shocks were delivered to perfusing rhythms. The rhythm did not change post-shock for $61 / 66$ (92.4\%) of these instances. In the five instances the rhythm changed from PEA postshock, one changed to VF and four to asystole.

\section{Discussion}

In cases with shockable rhythm 1 min after a defibrillation attempt, 99.1\% remained shockable (VF/VT) immediately before next possible shock. This high predictive value could be used as an argument for rhythm analysis earlier in the CPR-cycle to minimize the immediate pre-shock compression pause as other studies indicate that increased hands off intervals immediately pre-shock negatively influence shock success, ROSC and survival. ${ }^{3-7}$ In order to deliver an appropriate shock there are four important tasks that have to be done. ${ }^{1,2}$ First, chest compressions must be stopped in order to have a chest compression artefact free ECG. Second, ECG rhythm must be decided. Third, based on the ECG the defibrillator must be charged. Fourth, when the defibrillator is charged the shock can be delivered at the same time as the rescuer makes sure that "all clear". At present time all these manoeuvres will add time to the pre-shock pause that may influence termination of fibrillation negatively..$^{5-7}$ By performing the first and second task earlier the indication for defibrillation is already made when it is time for a shock. The defibrillator may be charged during chest compressions and this may save pre-shock pause. When the defibrillator is charged the rescuer make sure "all clear" and deliver the shock with a minimum of pre-shock chest compression pause. Cheskes et al. have recently documented that compressions during defibrillator charging shorten pre-shock pauses. ${ }^{16}$
Because $18.2 \%$ of 1880 non-shockable rhythms 1 min post-shock converted to a shockable rhythm after chest compressions, a nonshockable rhythm detected 1 min post-shock should result in a second pre-shock rhythm analysis as current guidelines recommend.

Of the 1529 shockable rhythms 1 min post shock, 99.1\% were shockable $1 \mathrm{~min}$ post-shock. There were three cases $(0.2 \%)$ in present study with conversion from VF/VT to ROSC during CPR without electrical defibrillation attempts. How this happened is speculation. Spontaneous termination of VF in patients with both myocardial infarction ${ }^{17,18}$ and without cardiac disease ${ }^{19}$ have been described. It could also be a result of administration of intravenous antiarrhythmic drugs. Studies have shown increased survival to hospital admission for shock resistant VF after administration of amiodarone $e^{20,21}$ and adrenaline. ${ }^{22}$ In the present study it is difficult to evaluate the medication effect because the timing of delivery of drugs was not recorded. Another possibility is that VF/VT was terminated by chest compressions. This has to our knowledge never been described before.

Our hypothesized approach to perform a rhythm analysis earlier in the CPR-cycle raises the risk of defibrillating a perfusing rhythm ( $0.2 \%$ in present study). The worst result of inappropriate shock in this vulnerable situation would be re-arrest. It has been documented that shocks delivered in the vulnerable period around the T-wave can induce VF/VT in humans. ${ }^{23,24}$ Ornato et al. have published a case report where a patient in sinus rhythm was shocked to VT with an external defibrillator and back to sinus rhythm again. ${ }^{25}$ Various antiarrhythmic drugs affect VF defibrillation threshold and may be protective regarding induction of VF by inappropriate shocks. ${ }^{26,27}$ This may be important to take into consideration when evaluating the possibility of inducing VF/VT by inappropriate shocks.

Kramer-Johansen et al. ${ }^{28}$ reported that six of 128 shocks inappropriately delivered to organized rhythms, all identified as PEA, converted to asystole and another six to VF/VT. In all these cases TTI and notions in the ambulance journal confirmed PEA. Both the present data and the data from Kramer-Johansen et al. indicates that shocking an organized rhythm will not terminate it in the great majority of instances ( $92 \%$ and $96 \%$, respectively). It may be debated if defibrillation of PEA, as described here, is comparable to shocking an organized pulse generating rhythm.

In the Lucas In Cardiac Arrest (LINC) trial Rubertsson et al. ${ }^{29}$ studied mechanical CPR in association with defibrillation during on-going chest compressions in comparison with regular CPR following 2005 ERC guidelines. They used a three-minute CPR cycle with rhythm analysis every $3 \mathrm{~min}$ and defibrillation $90 \mathrm{~s}$ into the CPR cycle and therefore no rhythm analysis in immediate association to the defibrillation attempts for the mechanical CPR group. The LINC trial showed no difference in four-hour survival between the mechanical CPR group (23.6\%) and the manual CPR group (23.7\%) (treatment difference, $-0.05 ; 95 \% \mathrm{CI},-3.3$ to 3.2$){ }^{29}$ They did not report pre-shock pause duration. This makes it impossible to interpret if their result reflects the effect of the mechanical chest compression device or their alternative CPR algorithm with defibrillation during chest compressions and no pre-shock rhythm analysis in immediate association with defibrillation attempts. 


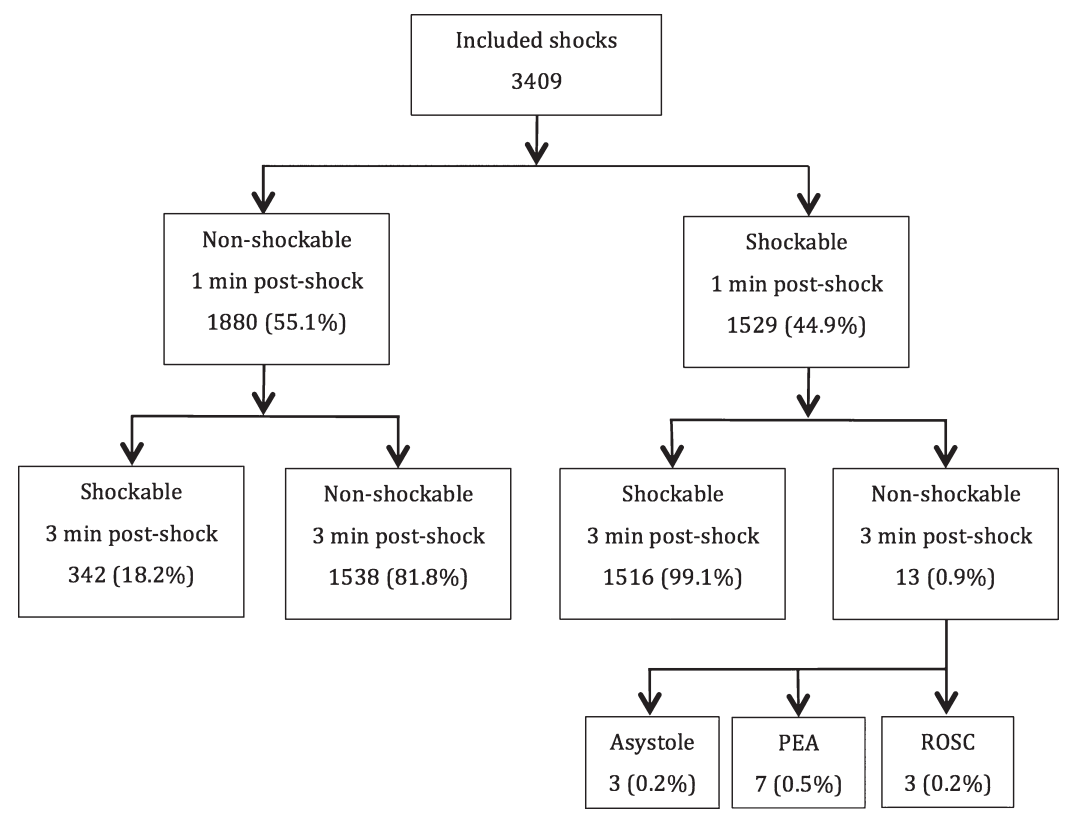

Fig. 1. Flow diagram of post-shock rhythms for included shocks.

Defibrillation during chest compressions do however represent another possible way to reduce pre-shock pauses and has shown to be safe in both CIRC and LINC trials. ${ }^{11,29}$ Some have suggested that hands-on defibrillation during manual chest compressions may be carried out in order to reduce pre-shock pauses, by using gloves or an isolating blanket. ${ }^{30,31}$ Concerns related to the ability of clinical examination gloves to protect the rescuer during simultaneous chest compressions and defibrillation have been raised. ${ }^{32,33}$ Deakin et al. concluded that nitrile clinical examination gloves do not provide adequate electrical insulation for the rescuer to safely undertake hands-on defibrillation. ${ }^{33}$ Therefore, Kerber in an editorial, asks for a cautious approach to achieve shorter pre-shock pauses by performing hands-on defibrillation with clinical examination gloves. ${ }^{34}$

There are several studies documenting increased defibrillation success rate with chest compressions prior to defibrillation attempts. ${ }^{3,4,6,8}$ This indicates that unnecessary chest compression pauses should be avoided. Eftestol et al. ${ }^{35}$ reported that CPR improved four out of five VF waveform defibrillation outcome predictors. The two most powerful predictors of ROSC in their study (AMSA and Prosc), improved significantly with CPR lasting longer than 3 min. ${ }^{35}$ In addition they reported that in patients with a predicted probability of ROSC after defibrillation above $25 \%$, this was halved after $20 \mathrm{~s}$ without $\mathrm{CPR} .{ }^{4}$ In a recent randomized clinical study overall survival was not higher with VF waveform analysis guidance whether to shock first or give 2 min of CPR first vs. a standard shock-first algorithm group. ${ }^{36}$ They do not report data on CPR quality, but if VF waveform score is a proxy for quality, the subgroup with improved VF waveform score after CPR had better outcome compared to those with decreased VF waveform score after CPR (ROSC $41.9 \%$ vs. $19.9 \%$, sustained ROSC $33.6 \%$ vs. $13.1 \%$, survival to admission $36.9 \%$ vs. $11.1 \%$, respectively, $p<0.001$ ).

Sell et al. demonstrated that pre-shock pauses less than $3 \mathrm{~s}$ were associated with increased ROSC. ${ }^{3}$ Cheskes et al. showed that pre-shock pause duration was independently associated with survival to hospital discharge. ${ }^{5}$ Covariates adjusted data showed that pre-shock pause $\geq 20 \mathrm{~s}$ was significantly associated with reduced survival to hospital discharge (OR, $0.47 ; 95 \% \mathrm{CI}, 0.27$ to 0.82 ) compared to pre-shock pause $<10 \mathrm{~s}$. On a continuous model, the odds for survival were reduced by $18 \%$ (OR, $0.82 ; 95 \% \mathrm{CI}, 0.73$ to 0.93 ) for every $5 \mathrm{~s}$ increase in pre-shock pause. ${ }^{5}$ Data from the ROC PRIMED trial documented that pre-shock pauses in chest compressions $<10 \mathrm{~s}$ were associated with both higher survival to hospital discharge (OR, 1.52; 95\% CI, 1.09 to 2.11 ) and higher survival with intact neurology (MRS $\leq 3)(\mathrm{OR}, 1.49 ; 95 \% \mathrm{Cl}, 1.05$ to 2.13$)$ compared to pre-shock pauses $\geq 20$ s. $^{7}$

Real time removal of compression artefacts from defibrillator ECGs using filtering technology could make it possible to perform rhythm analysis during continuous chest compressions. ${ }^{37}$ This technology is to our knowledge not yet available in commercial defibrillators.

Kalenda has noted that $\mathrm{ETCO}_{2}$ monitoring during CPR resulted in a sudden steep increase in $\mathrm{ETCO}_{2}$ when ROSC was achieved. ${ }^{38}$ Typically $\mathrm{ETCO}_{2}$ values during CPR range from $0 \mathrm{kPa}$ to $2.7 \mathrm{kPa}$, ${ }^{39}$ which is lower than the value of $4.6 \mathrm{kPa}$ to $5.9 \mathrm{kPa}$ during normal conditions. ${ }^{40,41}$ Monitoring and evaluating ETCO 2 values may help to identify patients with ROSC, and thus avoid delivering a shock to these patients.

\section{Limitations}

This is a retrospective analysis of data collected from the CIRC trial, not data collected prospectively for the specific purpose of this study. The gold standard would be a randomized trial comparing outcome and side effects between two CPR cycle strategies, one with an immediate pre-shock rhythm analysis and one with an earlier rhythm analysis, with survival as primary endpoint.

The large number of delivered shocks excluded from analysis is also a limitation, but reduces the chance of evaluation bias as all included ECG analyses were without compression artefacts.

CPR in this study was performed according to Norwegian CPR guidelines with three-minute CPR cycles ${ }^{12}$ and not the ERC and AHA recommended two-minute CPR cycles. The data are thus not immediately transferable, but this was not the purpose of this evaluation. It used the unique opportunity of studying artefact free ECG at two points in time during a CPR cycle as a model for cardiac rhythm development during on-going CPR. We have however no reason to believe that the results would be different with a two-minute CPR cycle. 


\section{Conclusion}

While a shockable rhythm one minute post-shock was still present immediately before the next possible defibrillation attempt in most cases, three patients regained a perfusing rhythm (ROSC) during CPR without defibrillation. We can therefore not recommend replacing pre-shock rhythm analysis with rhythm analysis earlier in the CPR cycle without additional information if the patient has achieved ROSC. More studies are needed to document if moving the immediate pre-shock rhythm analysis will increase the number of shocks delivered to organized rhythms and the consequences of such shocks, and/or if it will increase shock success and survival.

\section{Funding}

CIRC trial was funded by ZOLL Medical. Steinberg receives a research scholarship provided by the Norwegian Research Council. Olsen is partly funded by unrestricted grant from Norwegian Health Region South-East and partly by a research grant from ZOLL Medical to NAKOS.

Clinical Trial Registration: NCT 00597207 (http://clinicaltrials. gov/show/NCT00597207).

\section{Conflict of interest statement}

All authors' institutions received funding from ZOLL for their participation in the CIRC trial. Lars Wik is NAKOS representative in the Medical Advisory Board of PhysioControl. The authors have no other relevant financial conflicts of interest to report.

\section{Acknowledgements}

The authors would like to acknowledge the EMS providers who contributed to this study as well as other individuals who made this study possible. Thanks to the coordinators and monitors at each of the participating sites for their careful and persistent work with the data collection. A special thanks to Petter A Steen for valuable critique and revision of the manuscript.

\section{References}

1. Neumar RW, Otto CW, Link MS, et al. Part 8: adult advanced cardiovascular life support: 2010 American Heart Association Guidelines for Cardiopulmonary Resuscitation and Emergency Cardiovascular Care. Circulation 2010;122:S729-67.

2. Nolan JP, Soar J, Zideman DA, et al. European Resuscitation Council Guidelines for Resuscitation 2010 Section 1. Executive summary. Resuscitation 2010;81:1219-76.

3. Sell RE, Sarno R, Lawrence B, et al. Minimizing pre- and post-defibrillation pauses increases the likelihood of return of spontaneous circulation (ROSC). Resuscitation 2010;81:822-5

4. Eftestol T, Sunde K, Steen PA. Effects of interrupting precordial compressions on the calculated probability of defibrillation success during out-of-hospital cardiac arrest Circulation 2002:105:2270-3.

5. Cheskes S, Schmicker RH, Christenson J, et al. Perishock pause: an independent predictor of survival from out-of-hospital shockable cardiac arrest. Circulation 2011;124:58-66

6. Edelson DP, Abella BS, Kramer-Johansen J, et al. Effects of compression depth and pre-shock pauses predict defibrillation failure during cardiac arrest. Resuscitation 2006:71:137-45.

7. Cheskes S, Schmicker RH, Verbeek PR, et al. The impact of peri-shock pause on survival from out-of-hospital shockable cardiac arrest during the Resuscitation Outcomes Consortium PRIMED trial. Resuscitation 2014;85:336-42.

8. Gundersen K, Kvaløy JT, Kramer-Johansen J, Steen PA, Eftestøl T. Development of the probability of return of spontaneous circulation in intervals without chest compressions during out-of-hospital cardiac arrest: an observational study. BMC Med 2009;7:6, http://dx.doi.org/10.1186/1741-7015-7-6.

9. Christenson J, Andrusiek D, Everson-Stewart S, et al. Chest compression fraction determines survival in patients with out-of-hospital ventricular fibrillation. Circulation 2009;120:1241-7.

10. Rea T, Olsufka M, Yin L, Maynard C, Cobb L. The relationship between chest compression fraction and outcome from ventricular fibrillation arrests in prolonged resuscitations. Resuscitation 2014;85:879-84.
11. Wik L, Olsen JA, Persse D, et al. Manual vs. integrated automatic load-distributing band CPR with equal survival after out of hospital cardiac arrest. The randomized CIRC trial. Resuscitation 2014;85:741-8.

12. Lexow K, Sunde K. Why Norwegian 2005 guidelines differs slightly from the ERC guidelines. Resuscitation 2007;72:490-2.

13. Lerner EB, Persse D, Souders CM, et al. Design of the Circulation Improving Resuscitation Care (CIRC) trial: a new state of the art design for out-of-hospital cardiac arrest research. Resuscitation 2011;82:294-9.

14. Stecher FS, Olsen JA, Stickney RE, Wik L. Transthoracic impedance used to evaluate performance of cardiopulmonary resuscitation during out of hospital cardiac arrest. Resuscitation 2008;79:432-7.

15. Von Hippel PT. Regression with missing ys: an improved strategy for analyzing multiply imputed data. Sociol Methodol 2007;37:83-117.

16. Cheskes S, Common MR, Byers PA, Zhan C, Morrison LJ. Compressions during defibrillator charging shortens shock pause duration and improves chest compression fraction during shockable out of hospital cardiac arrest. Resuscitation 2014:85:1007-11.

17. Priest WM. Ventricular fibrillation recorded 10 hours before death from myocardial infarction. Lancet 1949;2:699.

18. Choquette G, Wasserman F, Lisker S, Bellet S. Spontaneous reversion of ventricular fibrillation to normal sinus rhythm in a case of acute myocardial infarction. Am Heart J 1956;51:455-9.

19. Dubner SJ, Gimeno GM, Elencwajg B, Leguizamon J, Tronge JE, Quinteiro R. Ventricular fibrillation with spontaneous reversion on ambulatory ECG in the absence of heart disease. Am Heart J 1983;105:691-3.

20. Kudenchuk PJ, Cobb LA, Copass MK, et al. Amiodarone for resuscitation after out-of-hospital cardiac arrest due to ventricular fibrillation. N Engl J Med 1999;341:871-8.

21. Dorian P, Cass D, Schwartz B, Cooper R, Gelaznikas R, Barr A. Amiodarone as compared with lidocaine for shock-resistant ventricular fibrillation. N Engl J Med 2002;346:884-90.

22. Herlitz J, Ekstrom L, Wennerblom B, Axelsson A, Bang A, Holmberg S. Adrenaline in out-of-hospital ventricular fibrillation. Does it make any difference? Resuscitation 1995;29:195-201

23. Lown B, Amarasin R, Neuman J. New method for terminating cardiac arrhythmias-use of synchronized capacitor discharge. JAMA-J Am Med Assoc 1962;182:548-\&.

24. Mazer CD, Greene MB, Misale PS, Newman D, Dorian P. Transcutaneous T wave shock: a universal method for ventricular fibrillation induction. Pacing Clin Electrophysiol 1997;20:2930-5.

25. Ornato JP, Shipley J, Powell RG, Racht EM. Inappropriate electrical countershocks by an automated external defibrillator. Ann Emerg Med 1992;21: 1278-81.

26. Anderson JL, Rodier HE, Green LS. Comparative effects of beta-adrenergic blocking drugs on experimental ventricular fibrillation threshold. Am J Cardiol 1983;51:1196-202.

27. Harumi K, Tsutsumi T, Sato T, Sekiya S. Classification of antiarrhythmic drugs based on ventricular fibrillation threshold. Am J Cardiol 1989;64:10J-4J.

28. Kramer-Johansen J, Edelson DP, Abella BS, Becker LB, Wik L, Steen PA. Pauses in chest compression and inappropriate shocks: a comparison of manual and semi-automatic defibrillation attempts. Resuscitation 2007;73:212-20.

29. Rubertsson S, Lindgren E, Smekal D, et al. Mechanical chest compressions and simultaneous defibrillation vs conventional cardiopulmonary resuscitation in out-of-hospital cardiac arrest: the LINC randomized trial. JAMA 2014:311:53-61.

30. Lloyd MS, Heeke B, Walter PF, Langberg JJ. Hands-on defibrillation: an analysis of electrical current flow through rescuers in direct contact with patients during biphasic external defibrillation. Circulation 2008;117:2510-4.

31. Yu T, Ristagno G, Li Y, Bisera J, Weil MH, Tang W. The resuscitation blanket: a useful tool for "hands-on" defibrillation. Resuscitation 2010;81:230-5.

32. Petley GW, Cotton AM, Deakin CD. Hands-on defibrillation: theoretical and practical aspects of patient and rescuer safety. Resuscitation 2012;83:551-6.

33. Deakin CD, Lee-Shrewsbury V, Hogg K, Petley GW. Do clinical examination gloves provide adequate electrical insulation for safe hands-on defibrillation? I: Resistive properties of nitrile gloves. Resuscitation 2013;84:895-9.

34. Kerber RE. Gloves to protect hands-and heart. Resuscitation 2013;84:859-60.

35. Eftestol T, Wik L, Sunde K, Steen PA. Effects of cardiopulmonary resuscitation on predictors of ventricular fibrillation defibrillation success during out-of-hospital cardiac arrest. Circulation 2004;110:10-5.

36. Freese JP, Jorgenson DB, Liu PY, Innes J, Matallana L, Nammi K, et al. Waveform analysis-guided treatment versus a standard shock-first protocol for the treatment of out-of-hospital cardiac arrest presenting in ventricular fibrillation: results of an international randomized, controlled trial. Circulation 2013;128:995-1002.

37. Aase SO, Eftestol T, Husoy JH, Sunde K, Steen PA. CPR artifact removal from human ECG using optimal multichannel filtering. IEEE Trans Biomed Eng 2000;47:1440-9.

38. Kalenda $Z$. The capnogram as a guide to the efficacy of cardiac massage. Resuscitation 1978;6:259-63.

39. Sanders AB. Capnometry in emergency medicine. Ann Emerg Med 1989;18:1287-90

40. Trillo G, von Planta M, Kette F. ETCO ${ }_{2}$ monitoring during low flow states: clinical aims and limits. Resuscitation 1994;27:1-8.

41. Hollinger CB, Hoyt JW. Capnography and respiratory monitoring. In: Textbook of Critical Care. 3rd ed. Philadelphia, PA: WB Saunders Company; 1995. p. 305-11. Chapter 36. 



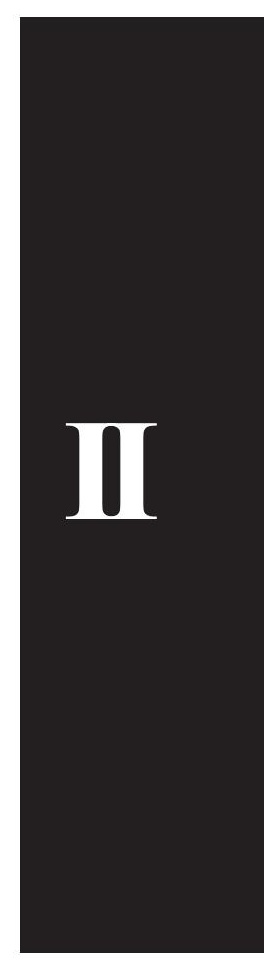



Clinical paper

\title{
Defibrillation success during different phases of the mechanical chest compression cycle ${ }^{\text {is }}$
}

\author{
Mikkel T. Steinberg a,b,*, Jan-Aage Olsen ${ }^{\mathrm{a}, \mathrm{b}}$, Cathrine Brunborg ${ }^{\mathrm{c}}$, David Persse ${ }^{\mathrm{d}}$, \\ Fritz Sterz ${ }^{\mathrm{e}}$, Michael Lozano Jr ${ }^{\mathrm{f}}$, Mark Westfallg,h, David T. Travis ${ }^{\mathrm{f}}$, E. Brooke Lerner ${ }^{\mathrm{i}}$, \\ Lars Wik ${ }^{\mathrm{b}}$ \\ a University of Oslo, Institute of Clinical Medicine, University of Oslo, Oslo, Norway \\ ${ }^{\mathrm{b}}$ Norwegian National Advisory Unit on Prehospital Emergency Medicine, Oslo University Hospital, Oslo, Norway \\ ' Oslo Centre for Biostatistics and Epidemiology, Research Support Services, Oslo University Hospital, Ullevål, Oslo, Norway \\ ${ }^{\mathrm{d}}$ Houston Fire Department and the Baylor College of Medicine, Houston, TX, United States \\ ${ }^{\mathrm{e}}$ Department of Emergency Medicine, Medical University of Vienna, Vienna, Austria \\ ${ }^{\mathrm{f}}$ Hillsborough County Fire Rescue, Tampa, FL, United States \\ ${ }^{g}$ Gold Cross Ambulance Service, Appleton Neenah-Menasha and Grand Chute Fire Departments, WI, United States \\ ${ }^{\mathrm{h}}$ Theda Clark Regional Medical Center, Neenah, WI, United States \\ i Department of Emergency Medicine, Medical College of Wisconsin, Milwaukee, WI, United States
}

\section{A R T I C L E I N F O}

\section{Article history:}

Received 28 July 2015

Received in revised form

16 December 2015

Accepted 25 January 2016

\section{Keywords:}

Cardiac arrest

Defibrillation

Mechanical chest compressions

\begin{abstract}
A B S T R A C T
Introduction: Animal studies indicate higher termination of VF/VT (TOF) rates after shocks delivered during the decompression phase of the compression cycle for manual and mechanical CPR. We investigated TOF for shocks delivered in different compression cycle phases during load distributing band (LDB) mechanical CPR in the CIRC trial.

Methods: Shocks were retrospectively categorized as delivered during the compression, decompression, or relaxation phase of LDB compressions using transthoracic impedance data. Shocks delivered when the LDB device was paused, were used as controls. The first shock and the first up-to-three shocks (first shocks plus shocks two and three if given) from patients with initial VF/VT and LDB CPR prior to shock were grouped according to compression cycle phase. TOF rates for these groups versus the control group were analyzed using logistic regression for first shocks and the general estimating equations (GEE) model for the up-to-three shocks. Adjustments were made for bystander CPR, witnessed arrest, defibrillator shock energy and transthoracic impedance.

Results: Among 244 first shocks and 685 up-to-three shocks TOF success rates were lower $(p<0.05$ and $p<0.02$ ) for shocks given during the compression phase ( $72 \%$ and $71 \%$ respectively) than for control shocks given during compression pauses ( $86 \%$ and $82 \%$ respectively). Decompression and relaxation phase shocks had TOF rates not different from the controls.

Conclusion: Shocks delivered in the compression phase of LDB chest compressions had lower TOF rates than shocks delivered while pausing the LDB device. More research is needed to see how defibrillation during chest compressions affect ROSC and survival.
\end{abstract}

(c) 2016 Elsevier Ireland Ltd. All rights reserved.

\footnotetext{
is A Spanish translated version of the abstract of this article appears as Appendix in the final online version at http://dx.doi.org/10.1016/j.resuscitation.2016.01.031. * Corresponding author at: Oslo University Hospital-Ullevål, Norwegian National Advisory Unit on Prehospital Emergency Medicine (NAKOS), Building 31, P.O. Box 4956 Nydalen, NO-0424 Oslo, Norway.

E-mail address: mikkelts@studmed.uio.no (M.T. Steinberg).
}

\section{Introduction}

Early cardiopulmonary resuscitation (CPR) and defibrillation are considered the most important factors for survival after sudden cardiac arrest. The interplay between chest compressions and defibrillation attempts in patients with shockable rhythms is probably important. The 2010 CPR guidelines of European Resuscitation Council (ERC) and American Heart Association (AHA) emphasize reducing chest compression interruptions as much as possible $^{1,2}$ based on studies showing negative effects of such 
pauses. ${ }^{3-6}$ Avoiding pre-shock pauses altogether could potentially increase the defibrillation success rate even further. This may be achieved with compressions given by a mechanical device or manually with appropriate safety gloves. ${ }^{7}$

Li et al. found higher termination of VF/VT $5 \mathrm{~s}$ post-shock (TOF) rate for shocks delivered during the decompression phase of the chest compression cycle compared with shocks delivered after a $2 \mathrm{~s}$ pre-shock chest compression pause for both manual and mechanical chest compressions in pigs. ${ }^{8,9}$ Three large randomized trials, the LINC, CIRC and PARAMEDIC trials, have evaluated two mechanical chest compression devices with defibrillation during ongoing chest compressions in the mechanical chest compression group (AutoPulse ${ }^{\circledR}$ Load Distributing Band [LDB], ZOLL Medical, Chelmsford, MA and LUCAS ${ }^{\circledR}$, Physio-Control, Redmond, WA). All three studies failed to show improved survival. ${ }^{10-12}$

Olsen et al. recently reported that TOF was lower with defibrillation during continuous LDB compressions for the first shock based on retrospective data from the CIRC trial. ${ }^{13}$ In an accompanying editorial Deakin pointed out that "defibrillation may be more successful in the relaxation phase of each compression cycle". ${ }^{14}$ The same was pointed out by Carron and Yersin regarding the LINC trial. ${ }^{15}$ Olsen et al. had not differentiated between compression cycle phases. We have now been able to define in what phases of the compression cycle the various shocks were given in the CIRC trial, and have therefore investigated the relation between TOF and shocks delivered in different LDB compression cycle phases on data from the CIRC trial. ${ }^{10}$

\section{Methods}

\section{Study population}

This is a retrospective study on electronical data from the CIRC trial. The multicentre CIRC trial was carried out in Houston TX, Hillsborough County FL and Fox Valley WY in the USA, in Nijmegen, the Netherlands, and in Vienna, Austria between March 5, 2009 and January 11,2011 . It was designed to compare manual chest compressions with integrated use of mechanical LDB-CPR in respect to survival to hospital discharge. ${ }^{10,16}$ CIRC followed international CPR Guidelines with the exception that the CPR cycle was 3 min. ${ }^{17}$ In the current paper shocks from CIRC patients with initial VF/VT and LDB-CPR prior to defibrillation were included for further investigation.

\section{Defibrillator data}

All participating sites used right subclavian and left apex pad positioning. The stick-on defibrillator pads were placed before the LDB device was deployed, and were in varying degrees covered by the LDB during chest compressions. The defibrillator energy protocol used was either fixed at $360 \mathrm{~J}$ for all shocks (one study site) or escalating (first shock $200 \mathrm{~J}$, second $200 \mathrm{~J}, 300 \mathrm{~J}$ or $360 \mathrm{~J}$ and third and subsequent $360 \mathrm{~J}$, four study sites). Transthoracic impedance (TTI), ECG signal and defibrillation attempt(s), were continuously recorded and stored by the defibrillators. ECG and TTI data from both LIFEPAK ${ }^{\circledR} 12,15$ and 500 (Physio-Control, Redmond, WA, USA) were analyzed using CODE-STAT ${ }^{\mathrm{TM}} 9.0$ software (Physio-Control, Redmond, WA, USA). ECG was analyzed for initial rhythm, pre-shock rhythm and $5 \mathrm{~s}$ post-shock rhythm. Preand post-shock rhythms were annotated by LW and JO, and 1002 post-shock rhythms (randomly selected by SPSS [Version 22.0, IBM SPSS Inc., Chicago, IL, USA]) were scored by both raters in order to document the inter-rater agreement on rhythm annotations. The rhythm annotation process is described in detail in a recent paper by Olsen et al. ${ }^{13}$

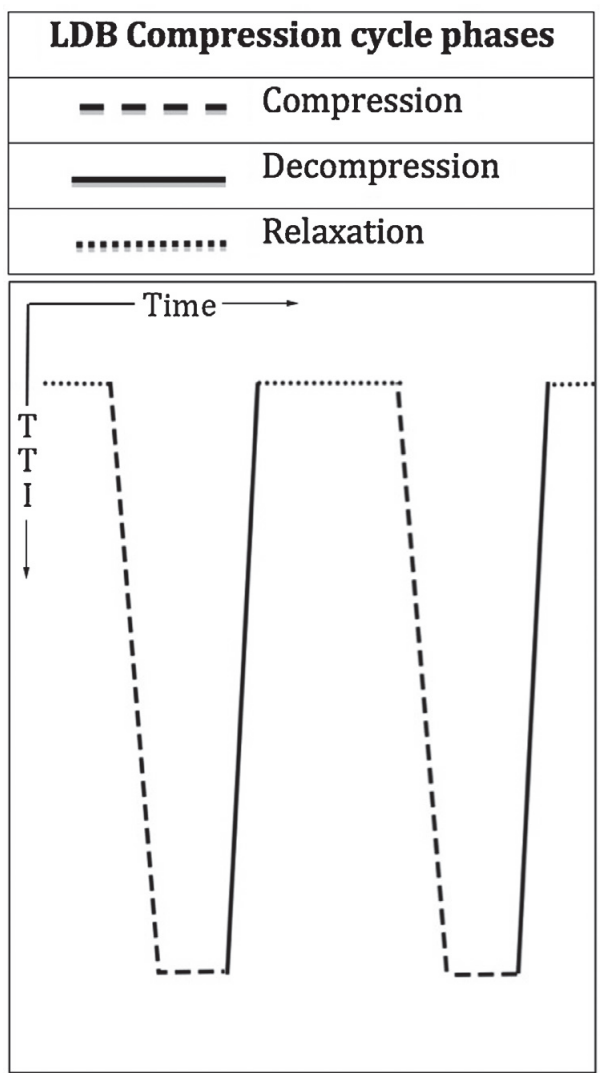

Fig. 1. Sketch of the LDB compression cycle phases defined by changes in transthoracic impedance (TTI). With right subclavian and left apex pads positioning, compression will be illustrated in CODE-STAT ${ }^{\mathrm{TM}}$ as a decreasing TTI graph. If the pads are interchanged, the polarity of the TTI signal will change and compressions will be illustrated as an increasing TTI graph.

\section{Annotation of shocks to LDB compression cycle phases}

The chest compression-decompression cycle of the LDB-device consists of the following phases (Fig. 1): compression phase (the load-distributing band tightens and holds tight), decompression phase (the load-distributing band loosens, no active decompression) and the relaxation phase (load-distributing band stays loose). The compression phase in our study is similar to what is referred to as the downstroke phase, and the decompression phase is similar to the upstroke phase in the pig studies conducted by Li et al. ${ }^{8,9}$ With the LDB-device attached the rescuers should defibrillate during continuous chest compressions according to protocol.

TTI data graphed over time can identify when chest compressions are given. ${ }^{18}$ The LDB-device compresses the chest at a constant rate of $80 \mathrm{~min}^{-1}$, producing a TTI graph of distinctive regularity and morphology, which enables identification of the different LDB compression cycle phases. Based on this and data from Zoll Medical on the LDB device work cycle, we made a transparent grid with the LDB work cycle printed on it, and scaled it to the same calibration as CODE-STAT ${ }^{\mathrm{TM}}$ - displaying ECG and TTI at $50 \mathrm{~mm} / \mathrm{s}$. Physio-Control provided data on the time interval from when the shock is marked as delivered in the CODE-STAT ${ }^{\mathrm{TM}}$ software, to when the shock is actually delivered by the different defibrillators. By using these data and aligning the transparent grid to the CODE-STAT ${ }^{\mathrm{TM}}$ TTI graph we determined in which part of the LDB compression cycle each shock was delivered (Fig. 2). For each patient the first shock plus shocks 2 and 3 if given, were analyzed. One rater (MS) assigned the phase for all shocks, and 

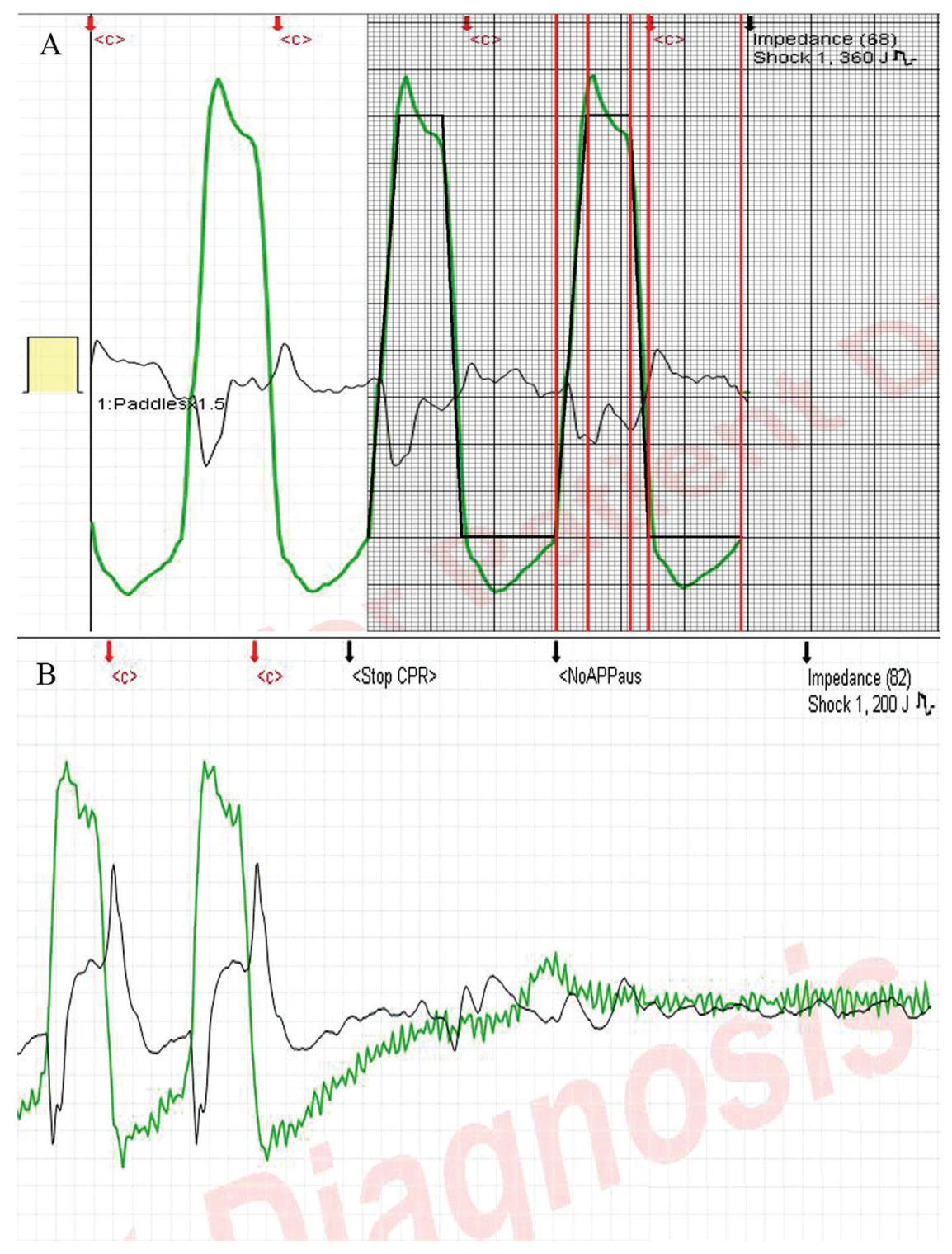

Fig. 2. CODE-STAT TM graph of ECG (black) and LDB chest compressions displayed with a TTI graph (green). (A) Displays a shock during LDB compressions and the right part of the figure includes a transparent with the printed LDB compression cycle phases. (B) Displays a control shock delivered in a chest compression pause. (For interpretation of the references to colour in this figure legend, the reader is referred to the web version of this article.)

a second blinded rater assigned a random $10 \%$ sample selected by SPSS, and inter-rater agreement was determined. Shocks delivered to rhythms other than VF/VT, with missing TTI or ECG signal, or if we were unable to determine in which phase of the LDB compression cycle the shock was delivered, were excluded. A number of first shocks were given after manual compressions and before the LDB-device had been deployed and were therefore also excluded.

In order to mimic the methods used by Li et al. ${ }^{8,9}$ we defined a control group of shocks from the same patient population with initial VF/VT and LDB CPR prior to shock. The controls were however defibrillated in a compression pause due to protocol violation (LDB device stopped by the rescuer in order to defibrillate). In the up-to-three shocks group, controls represented shocks and not patients. Therefore one patient could contribute with shocks to both control group and different chest compression cycle phases, depending on the timing of the shock delivery in relation to chest compressions.

\section{Statistical analyses}

Statistical analyses were performed using SPSS 22.0 (IBM SPSS Inc., Chicago, IL, USA) and Stata ${ }^{\circledR} 14$ (StataCorp LP, College Station, TX, USA). Two analyses were conducted. The first included only the first shock given to each patient. The second also included all first shocks, but added all second and third shocks, if given. The shocks were grouped according to the three compression-decompression phases or the control group, as described above. As all shocks were not necessarily given in the same compression phase in a patient, two or three shocks in a single patient could be assigned to different study groups. Outcome was defibrillation success defined as termination of fibrillation (VF/VT) $5 \mathrm{~s}$ post-shock to any rhythm but VF/VT; TOF. ${ }^{1,2}$ Logistic regression was used to compare TOF rates between LDB chest compression cycle phases and the control group in the first shock analysis. Adjustments were made for witnessed arrest, bystander CPR, defibrillator shock energy and transthoracic impedance. To account for possible correlations between 
the repeated measures in the up-to three shocks group, the general estimating equations (GEE) model with exchangeable matrix structure was used to study the association between TOF rates in the different LDB chest compression cycle phases and the control group. Defibrillator shock energy and transthoracic impedance were identified as possible patient independent confounding variables for defibrillation success, and were adjusted for. Results are presented as Odds ratio with $95 \% \mathrm{CI}$ and $p<0.05$ was considered significant.

Inter-rater agreement was assessed by unweighted Kappa statistics. Kappa values $>0.81$ was considered as excellent agreement.

\section{Results}

In the CIRC trial, 1657 patients received one or more shocks. Of these, 916 had an initial rhythm of VF/VT, and among these 370 received LDB-CPR prior to one or more defibrillation attempts. These patients received 1249 shocks. Shocks included for analysis are presented in Fig. 3 and shock characteristics for the different phases and controls in Table 1 . The TOF success rate was significantly lower for the first shock when delivered in the compression phase of the LDB compression cycle than for control shocks delivered in a LDB compression pause (14\% reduction, $72 \%$ vs. $86 \%$ ). The same results were found for the first up-to-three shocks group ( $11 \%$ reduction, $71 \%$ vs. $82 \%$ ). These results remained unchanged after adjusting for witnessed arrest and bystander CPR (first shock group only), defibrillator shock energy and transthoracic impedance. There were no differences in TOF success rates between the decompression or relaxation phase and the controls in either first shock or up-to-three shocks analysis (Table 2).

The pre-shock pause in the first shock control group was median 12 s with $5-20$ s quartiles (0-9 s: $46 \%, 10-19$ s: $29 \%, 20-29$ s: $18 \%$, $>30$ s: $8 \%$ ). For the up-to-three shocks control group the pre-shock pause was median $13 \mathrm{~s}$ with $4-20 \mathrm{~s}$ quartiles $(0-9 \mathrm{~s}$ : $40 \%, 10-19 \mathrm{~s}$ : $33 \%, 20-29$ s: $15 \%,>30$ s: $12 \%)$.

The Kappa value was 0.87 (95\% CI, $0.84-0.90, p<0.001)$ for the inter-rater agreement assessment on post-shock rhythm annotations and $0.93(95 \% \mathrm{CI}, 0.86-1.00, p<0.001)$ for the LDB compression phase annotations.

The LDB-device unintentionally stopped delivering chest compressions post-shock in $31 \%$ of the 422 defibrillation attempts delivered during LDB chest compressions in the up-to-three shocks group. This was verified by TTI graphs and notions in the case report forms based on ambulance records. It occurred significantly more often when the shock was delivered in the relaxation phase of the LDB compression cycle (compression $17 \%$, decompression $16 \%$, relaxation $49 \%, p<0.001)$.

\section{Discussion}

In this first clinical evaluation of defibrillation attempts during different phases of LDB chest compressions, TOF rates were lower for shocks delivered in the compression phase than in controls with a pre-shock chest compression pause. The present clinical data do not lend support to findings in pigs of higher success rate for shocks delivered in the decompression phase of the chest compression cycle than with a pre-shock pause. ${ }^{8,9}$ A species difference cannot be excluded. It should be noted that the pre-shock pause in the porcine studies was set at $2 \mathrm{~s}$, whereas it in the present paper was longer (median $12 \mathrm{~s}$ in first shock controls and $13 \mathrm{~s}$ in up-tothree shock controls) which represent a clinically more realistic scenario.

In the present study the pre-shock pause was zero for all shocks delivered during chest compressions to the different chest compression cycle phases, but not for the controls. Therefore the results of our analyses should be considered a combined result of both zero pre-shock pause and defibrillation in a specific phase of the LDB chest compression cycle. We have not been able to separate these results in a meaningful way because of the retrospective nature of the study. If we were to select cases for the control groups with a pre-shock pause less then i.e. $2 \mathrm{~s}$, there would not be enough shocks available for a meaningful analysis ( 4 and 11 shocks in first and up-to-three shocks groups, respectively). This may however strengthen the indication that defibrillation during the compression phase of the LDB chest compression cycle reduces TOF rates, as we would expect the effect of zero pre-shock pause alone to result in favourable TOF rates for the compression phase, compared with controls with longer pre-shock pauses as documented earlier. ${ }^{6}$

We do not know the physiological reasons for our results, but hypothesize that chest compressions influence defibrillation success via changes in the position and shape of the heart. Transesophageal echocardiography in humans has demonstrated that chest compressions reduce the size of all cardiac chamber in addition to distorting the ascending aorta and superior vena cava. ${ }^{19}$ The orientation and placement of external defibrillator pads are based on the normal anatomical location of the heart inside the chest. The changes in intrathoracic configurations and consequently changing the angle of the electrodes with chest compressions may influence the approximate $4 \%$ of the current that reaches the heart during shock, ${ }^{20}$ and potentially thereby the TOF rate.

It is also possible that increased physical strain on the heart from simultaneous compressions and defibrillation could reduce the TOF success rate for shocks delivered in the compression phase. Li et al. found that the combined force of the chest compression and shockinduced tetanic contraction of the animal almost doubled when the shock was delivered in the compression phase of the chest compression cycle compared to the decompression or decompressed (relaxation) phase. ${ }^{8,9}$ The force of chest compressions to tetanic contractions was not measured in the CIRC trial.

Ideally the first shock group should have been larger enabling not only TOF analysis, but also analysis of ROSC or longer-term survival. Even though 4231 patients were included in CIRC, there were too few patients with initial VF/VT and pre-shock LDB-CPR for meaningful ROSC analysis since ROSC was too infrequent (3 incidences in the first shock analysis and 10 in the up to three shocks analysis). The first up-to-three shocks analysis was added to increase the number of early resuscitation data, avoiding later shocks as early and late resuscitation represents two different scenarios for defibrillation success. Koster et al. demonstrated that TOF decreases significantly from the first to the fifth shock in patients with frequent refibrillations. ${ }^{21}$ Weisfeldt and Becker suggested that defibrillation should be emphasized in what they labelled an initial electrical phase (up to approximately 4 min of cardiac arrest) and a circulatory phase (up to approximately $10 \mathrm{~min}$ of cardiac arrest), but might not be sufficient in a following metabolic phase (after approximately $10 \mathrm{~min}$ of cardiac arrest).22

The CIRC protocol stated that defibrillation during chest compression should be attempted with the LDB-device maximally compressed, assuming that provider-device delay would cause most shocks to be delivered during the decompression phase. Shocks were however delivered apparently random throughout the compression-decompression cycle, with only $14 \%$ delivered in the decompression phase. Synchronization of shock with a specific compression cycle phase in future research will require automatic synchronization between the mechanical chest compression device and the defibrillator.

Shocks during chest compressions caused frequent unintentional stops in the LDB device after defibrillation. These stops occurred most often after shocks delivered during the relaxation phase of the LDB compression cycle. We hypothesize that the stops 


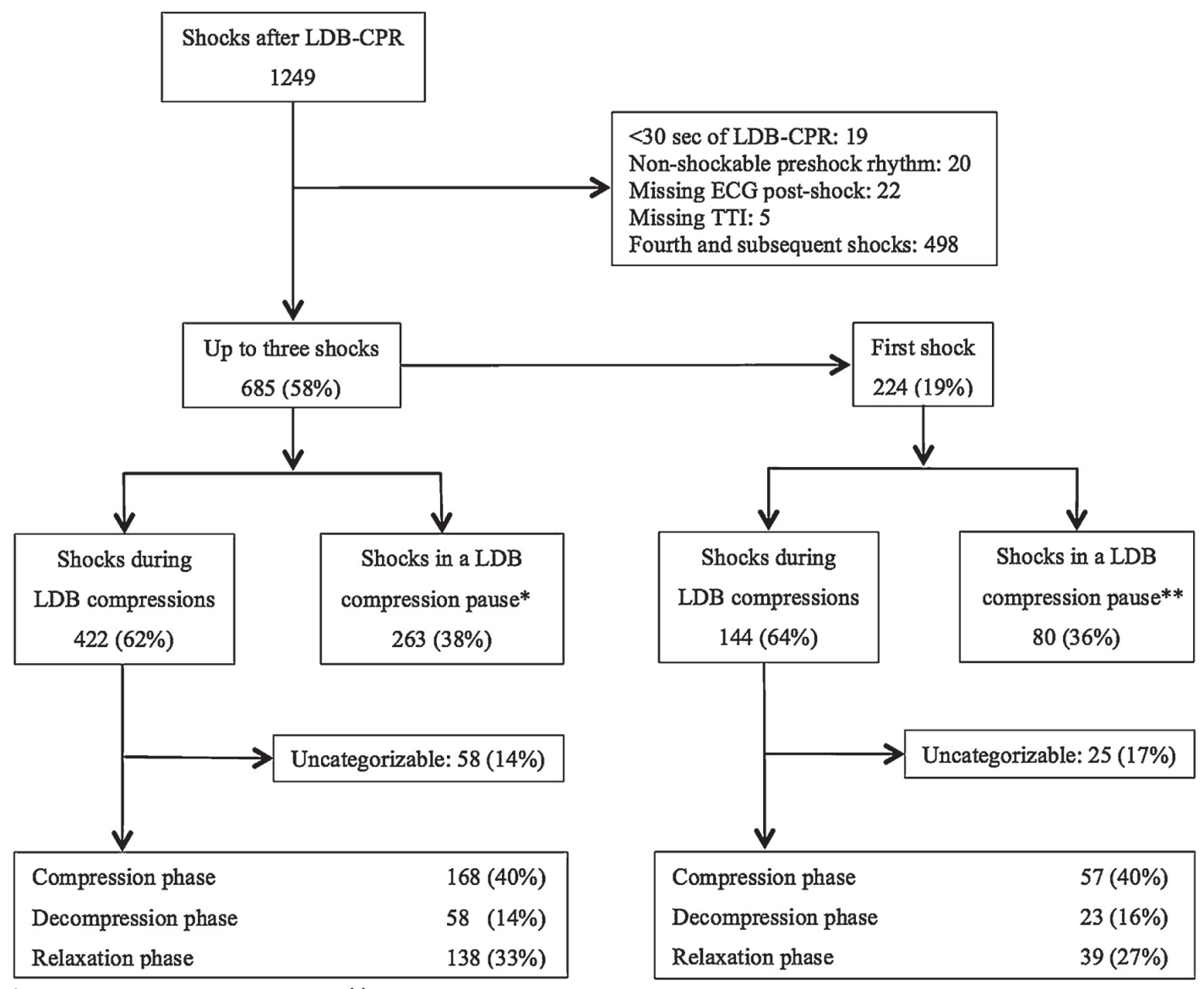

${ }^{*}$ Up to three shocks control group ${ }^{* *}$ First shock control group

Fig. 3. Flow-chart showing all shocks from the 370 included patients and how they are distributed to the different phases of the LDB compression cycle.

Table 1

Shock characteristics for the first shock group and up-to-three shocks group presented in medians and quartiles. Witnessed arrest and bystander CPR are only presented for the first shock group since patients could contribute with shocks to different phases in the up-to-three shocks group.

\begin{tabular}{llll}
\hline First shock group & Control, $n=80$ & Compression, $n=57$ & Decompression, $n=23$ \\
\hline Joules & $360,200-360$ & $360,200-360$ & $200,200-360$ \\
Transthoracic impedance & $86,67-104$ & $77,65-106$ & $91,71-103$ \\
Witnessed arrest (missing $n=9)$ & $58(73 \%)$ & $36(63 \%)$ & $20(87 \%)$ \\
Bystander CPR (missing $n=8)$ & $47(59 \%)$ & $28(49 \%)$ & $16(70 \%)$ \\
Up-to-three shocks group & Control, $n=263$ & Compression, $n=168$ & $200-360$ \\
Joules & $360,200-360$ & $360,200-360$ & Decompression, $n=58$ \\
Transthoracic impedance & $85,69-105$ & $80,69-106$ & $360,200-360$ \\
\hline
\end{tabular}

Transthoracic impedance was measured by the defibrillator before shock and not necessary in the specific chest compression cycle phase as indicated in this table. The impedance value is however the value used for defibrillator shock energy calculations for the shocks delivered in these specific chest compression cycle phases.

occurred due to safety technology built into the device. In the relaxation phase the band is in its loosest position, enabling tetanic body contractions caused by the shock to move some patients' bodies on the LDB device. When this is recognized by LDB device sensors, the system stops for safety reasons. By protocol, the rescuers were instructed to resume compressions manually while resetting the LBD device in these instances. As this occurred after defibrillation it is unlikely to influence defibrillation success defined as no shockable rhythm $5 \mathrm{~s}$ post-shock. A further exploration of this was not within the scope of the present paper. Until this issue is solved we believe crews attempting defibrillation without stopping the LDB device must be aware of these unintentional stops and if needed provide manual chest compression and restart the device as soon as possible.
In a recent analysis of a subset of patients from the LINC trial, Esibov et al. found no significant TOF differences between shocks delivered in chest compression pauses $(39 / 51,76 \%)$ and shocks during chest compressions delivered by the Lucas device (68/99, 69\%, Chi Square test, $p=0.32) .^{23}$ This is also a retrospective study with a lower number of shocks available for analysis, and one might speculate that this difference could be significant if a higher proportion of the data from the LINC trial were available for analysis.

\section{Limitations}

This post hoc investigation was carried out on data from the clinical trial CIRC, and the data were not prospectively collected for the purpose of this study. 
Table 2

The first shock analysis utilizing logistic regression to compare TOF rates between the LDB compression cycle phases and the control group. Unadjusted and adjusted results are presented as OR with 95\% Cl. Adjustment were made for witnessed arrest, bystander CPR, defibrillator shock energy and transthoracic impedance.

\begin{tabular}{|c|c|c|c|c|c|}
\hline First shock & TOF (\%) & Unadjusted OR $(95 \% \mathrm{CI})$ & $p$-value & Adjusted OR (95\% CI) & $p$-value \\
\hline Control group & $69 / 80(86)$ & & & & \\
\hline Compression & $41 / 57(72)$ & $0.41(0.17-0.97)$ & 0.04 & $0.42\left(0.17-1.00^{*}\right)$ & $0.05^{* *}$ \\
\hline Decompression & $18 / 23(78)$ & $0.57(0.18-1.87)$ & 0.36 & $0.56(0.17-1.83)$ & 0.34 \\
\hline Up to three shocks & TOF $(\%)$ & Unadjusted OR (95\% CI) & $p$-value & Adjusted OR (95\% CI) & $p$-value \\
\hline Control group & $216 / 263(82)$ & & & & \\
\hline Compression & $119 / 168(71)$ & $0.55(0.34-0.89)$ & 0.02 & $0.56(0.34-0.91)$ & 0.02 \\
\hline Decompression & $45 / 58(78)$ & $0.70(0.35-1.39)$ & 0.31 & $0.71(0.36-1.39)$ & 0.32 \\
\hline Relaxation & $103 / 138(75)$ & $0.67(0.40-1.14)$ & 0.14 & $0.66(0.38-1.13)$ & 0.13 \\
\hline
\end{tabular}

The up-to-three shocks analysis utilizing the general estimating equations (GEE) model with exchangeable matrix structure was used to study the association between TOF rates in the different LDB chest compression cycle phases and the control group. Unadjusted and adjusted results are presented as OR with $95 \% \mathrm{Cl}$. Adjustment were made for defibrillator shock energy and transthoracic impedance.

${ }^{*}$ Exact OR 95\% CI upper limit $=0.995$.

Ex Exact $p=0.049$.

Although the method used to retrospectively assign defibrillation attempts to different LDB compression cycle phases is new, there was excellent inter-rater agreement with high Kappa scores, indicating that this was likely an appropriate approach. Unfortunately, there were a relatively high number of shocks that we were unable to assign to one of the LDB compression phases (17\% first shocks, $14 \%$ in the up-to-three shocks group). This was likely random, but we cannot rule out the possibility of bias.

The statistical analyses were not adjusted for multiple comparisons. The possibility of type I and type II errors is always present, and the risk of type II error is increased by making multiple comparisons. ${ }^{24,25}$

This is a study of the "electrical outcome" of shocks delivered in different LDB compression cycle phases, not on patient outcome. The first shock analyses were based on independent patient observations, but not the up-to-three shocks analyses where one patient could contribute shocks to several LDB compression cycle phase groups. We have therefore adjusted for multiple observations per patient in the up-to-three shocks analysis by using the GEE model.

Analysing only patients with initial VF/VT may be criticized, but aligns well with recent studies on defibrillation success. ${ }^{3-6}$ These patients represent a more homogeneous group compared to patients with initial non-shockable rhythms, enabling easier comparisons between studies.

These results based on the LDB mechanical chest compression device cannot automatically be extrapolated to other mechanical chest compression devices. The LDB device compresses the chest over a larger area compared to a piston based device, and thus may have a different influence on how the electrical current reaches the heart.

\section{Conclusion}

The TOF rate was lower for defibrillation attempts during the compression phase of the LDB compression cycle than for control shocks delivered in a chest compression pause. The mechanism of this effect requires further investigation and more research is needed to study the timing of defibrillation during continuous mechanical chest compressions, and its relationship to ROSC and survival.

\section{Funding sources}

CIRC trial was funded by ZOLL Medical. Steinberg receives a research scholarship provided by the Norwegian Research Council. Olsen is partly funded by unrestricted grant from Norwegian
Health Region South-East and partly by a research grant from ZOLL Medical to NAKOS.

\section{Disclosures}

All authors' institutions received funding from ZOLL for their participation in the CIRC trial. LW represents NAKOS in the Medical Advisory Board of Physio-Control.

\section{Conflict of interest statement}

The authors have no other relevant financial conflicts of interest to report.

\section{Acknowledgments}

We acknowledge the EMS providers as well as other individuals who made this study possible. We would also like to thank the coordinators and monitors at the participating sites for their persistent and careful work with the data collection. A special thanks to Petter A Steen for valuable critique and revision of the manuscript.

\section{References}

1. Field JM, Hazinski MF, Sayre MR, et al. Part 1: Executive Summary 2010 American Heart Association Guidelines for Cardiopulmonary Resuscitation and Emergency Cardiovascular Care. Circulation 2010;122:S640-56.

2. Nolan JP, Soar J, Zideman DA, et al. European Resuscitation Council Guidelines for Resuscitation 2010 Section 1 Executive summary. Resuscitation 2010;81:1219-76.

3. Cheskes S, Schmicker RH, Christenson J, et al. Perishock pause: an independent predictor of survival from out-of-hospital shockable cardiac arrest. Circulation 2011;124:58-66

4. Cheskes S, Schmicker RH, Verbeek PR, et al. The impact of peri-shock pause on survival from out-of-hospital shockable cardiac arrest during the Resuscitation Outcomes Consortium PRIMED trial. Resuscitation 2014;85:336-42.

5. Sell RE, Sarno R, Lawrence B, et al. Minimizing pre- and post-defibrillation pauses increases the likelihood of return of spontaneous circulation (ROSC). Resuscitation 2010;81:822-5.

6. Edelson DP, Abella BS, Kramer-Johansen J, et al. Effects of compression depth and pre-shock pauses predict defibrillation failure during cardiac arrest. Resuscitation 2006;71:137-45.

7. Deakin CD, Thomsen JE, Lofgren B, Petley GW. Achieving safe hands-on defibrillation using electrical safety gloves - a clinical evaluation. Resuscitation 2015:90:163-7.

8. Li Y, Yu T, Ristagno G, et al. The optimal phasic relationship between synchronized shock and mechanical chest compressions. Resuscitation 2010;81:724-9.

9. Li Y, Wang H, Cho JH, et al. Defibrillation delivered during the upstroke phase of manual chest compression improves shock success. Crit Care Med 2010;38:910-5.

10. Wik L, Olsen JA, Persse D, et al. Manual vs. integrated automatic load-distributing band CPR with equal survival after out of hospital cardiac arrest. The randomized CIRC trial. Resuscitation 2014;85:741-8. 
11. Rubertsson S, Lindgren E, Smekal D, et al. Mechanical chest compressions and simultaneous defibrillation vs conventional cardiopulmonary resuscitation in out-of-hospital cardiac arrest: the LINC randomized trial. JAMA 2014;311:53-61.

12. Perkins GD, Lall $R$, Quinn $T$, et al. Mechanical versus manual chest compression for out-of-hospital cardiac arrest (PARAMEDIC): a pragmatic, cluster randomised controlled trial. Lancet 2014;385:947-55.

13. Olsen JA, Brunborg C, Steinberg M, et al. Pre-shock chest compression pause effects on termination of ventricular fibrillation/tachycardia and return of organized rhythm within mechanical and manual cardiopulmonary resuscitation. Resuscitation 2015:187:3-570.

14. Deakin CD. One swallow does not make a summer. Resuscitation 2015;93: A7-8.

15. Carron PN, Yersin B. Cardiopulmonary resuscitation with mechanical chest compressions and simultaneous defibrillation. JAMA 2014;311:2234.

16. Lerner EB, Persse D, Souders CM, et al. Design of the Circulation Improving Resuscitation Care (CIRC) Trial: a new state of the art design for out-of-hospital cardiac arrest research. Resuscitation 2011:82:294-9.

17. Lexow K, Sunde K. Why Norwegian 2005 guidelines differs slightly from the ERC guidelines (vol 72, pg 490, 2007). Resuscitation 2007;73:494.
18. Stecher FS, Olsen JA, Stickney RE, Wik L. Transthoracic impedance used to evaluate performance of cardiopulmonary resuscitation during out of hospital cardiac arrest. Resuscitation 2008;79:432-7.

19. Pell AC, Guly UM, Sutherland GR, Steedman DJ, Bloomfield P, Robertson C. Mechanism of closed chest cardiopulmonary resuscitation investigated by transoesophageal echocardiography. J Accid Emerg Med 1994;11:139-43.

20. Lerman BB, Deale OC. Relation between transcardiac and transthoracic current during defibrillation in humans. Circ Res 1990;67:1420-6.

21. Koster RW, Walker RG, Chapman FW. Recurrent ventricular fibrillation during advanced life support care of patients with prehospital cardiac arrest. Resuscitation 2008;78:252-7.

22. Weisfeldt ML, Becker LB. Resuscitation after cardiac arrest: a 3-phase timesensitive model. JAMA 2002;288:3035-8.

23. Esibov A, Banville I, Chapman FW, Boomars R, Box M, Rubertsson S. Mechanical chest compressions improved aspects of CPR in the LINC trial. Resuscitation 2015;91:116-21.

24. Rothman KJ. No adjustments are needed for multiple comparisons. Epidemiology 1990;1:43-6.

25. Perneger TV. What's wrong with Bonferroni adjustments. BMJ 1998;316: 1236-8. 



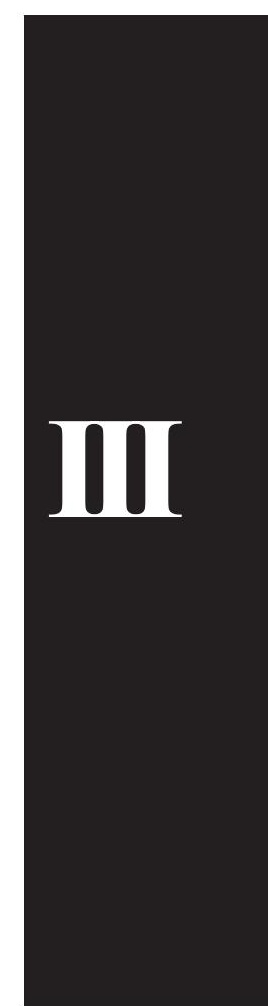





\title{
Haemodynamic outcomes during piston-based mechanical CPR with or without active decompression in a porcine model of cardiac arrest
}

\author{
Mikkel T. Steinberg ${ }^{1,2 *}$, Jan-Aage Olsen ${ }^{2,3}$, Morten Eriksen $^{4}$, Andres Neset $^{5}$, Per Andreas Norseng ${ }^{4}$,
} Jo Kramer-Johansen ${ }^{1,2}$, Bjarne Madsen Hardig ${ }^{6}$ and Lars Wik ${ }^{2}$

\begin{abstract}
Background: Experimental active compression-decompression (ACD) CPR is associated with increased haemodynamic outcomes compared to standard mechanical chest compressions. Since no clinically available mechanical chest compression device is capable of ACD-CPR, we modified the LUCAS 2 (Physio-Control, Lund, Sweden) to deliver ACD-CPR, hypothesising it would improve haemodynamic outcomes compared with standard LUCAS CPR on pigs with cardiac arrest.

Methods: The modified LUCAS delivering $5 \mathrm{~cm}$ compressions with or without $2 \mathrm{~cm}$ active decompression above anatomical chest level was studied in a randomized crossover design on 19 Norwegian domestic pigs. VF was electrically induced and untreated for 2 min. Each pig received ACD-CPR and standard mechanical CPR in three 180-s. phases. We measured aortic, right atrial, coronary perfusion, intracranial and oesophageal pressure, cerebral and carotid blood flow and cardiac output. Two-sided paired samples t-test was used for continuous parametric data and Wilcoxon test for non-parametric data. $P<0.05$ was considered significant.

Results: Due to injuries/device failure, the experimental protocol was completed in nine of 19 pigs. Cardiac output (I/min, median, (25, 75-percentiles): $1.5(1.1,1.7)$ vs. $1.1(0.8,1.5), p<0.01)$, cerebral blood flow (AU, 297 vs. 253, mean difference: $44,95 \% \mathrm{Cl}$; 14-74, $p=0.01)$, and carotid blood flow (I/min, median, (25, 75-percentiles): 97 (70, 106) vs. 83 $(57,94), p<0.01)$ were higher during ACD-CPR compared to standard mechanical CPR. Coronary perfusion pressure (CPP) trended towards higher in end decompression phase.
\end{abstract}

Conclusion: Cardiac output and brain blood flow improved with mechanical ACD-CPR and CPP trended towards higher during end-diastole compared to standard LUCAS CPR.

Keywords: Cardiac arrest, Active decompression, Experimental porcine model

\section{Background}

Mechanical chest compressions during cardiac arrest have improved hemodynamic variables in porcine and human studies and been documented to be safe with equal survival rates to high quality manual chest compressions during OHCA [1-6]. The use of active compression-

\footnotetext{
* Correspondence: mikkelts@medisin.uio.no

${ }^{1}$ Institute of Clinical Medicine, University of Oslo, Oslo, Norway

${ }^{2}$ Norwegian National Advisory Unit on Prehospital Emergency Medicine, Oslo University Hospital, Oslo, Norway

Full list of author information is available at the end of the article
}

decompression CPR (ACD-CPR), with active decompression to a higher level than the normal anatomical level, has showed promising results compared to standard chest compressions in both animals and humans [7-17]. These studies utilized either large mechanical power driven customized devices or a handheld device (CardioPump) to deliver ACD-CPR [7-17]. The mechanical devices used in the animal lab were impossible to bring into the field, and use of the handheld device did not deliver the same level of standardization and continuity as mechanical devices, resulting in lower fractions of ACD-CPR adhering to

() The Author(s). 2018 Open Access This article is distributed under the terms of the Creative Commons Attribution 4.0 International License (http://creativecommons.org/licenses/by/4.0/), which permits unrestricted use, distribution, and reproduction in any medium, provided you give appropriate credit to the original author(s) and the source, provide a link to the Creative Commons license, and indicate if changes were made. The Creative Commons Public Domain Dedication waiver (http://creativecommons.org/publicdomain/zero/1.0/) applies to the data made available in this article, unless otherwise stated. 
guidelines [18, 19]. The handheld device has been studied extensively, both alone and combined with an impedance threshold device (ITD). Systematic reviews conclude that neither manual ACD-CPR nor the ITDdevice during manual CPR improve long time survival. However, Aufderheide et al. demonstrated increased survival when combining the two techniques [20-23].

No commercially available automatic mechanical chest compression device has so far been able to perform ACD-CPR. Such a device would be of both academic and clinical interest since a mechanical device can enable consistent high quality ACD-CPR independent of rescuer fatigue. We hypothesized that the commercially available piston-based battery/mains powered mechanical chest compression device LUCAS 2 (PhysioControl/Jolife AB, Lund Sweden) modified to deliver ACD-CPR, would improve hemodynamic parameters during cardiac arrest in pigs compared with standard mechanical compressions delivered by LUCAS 2 .

\section{Methods \\ Study design}

This study compared mechanical CPR during ventricular fibrillation (VF) with piston-based chest compressions (LUCAS 2) with and without active decompression to $2 \mathrm{~cm}$ above normal anatomical level. Each pig served as its own control in cross over design. After surgery and preparation, but before induction of VF, pigs were randomized by drawing one of 19 envelopes where the sequence of the CPR techniques was written. A balanced design was achieved with each CPR technique performed once or twice on each pig the same number of times (intervention-control-intervention, or controlintervention-control).

The experiments were carried out in accordance with "Regulations on Animal Experimentation" under The Norwegian Animal Welfare Authority Act and approved by Norwegian Animal Research Authority (FOTS-ID 4931).

\section{Animal preparation and instrumentation}

Healthy Norwegian domestic pigs of both genders fasted eight hours prior to the experiment, but had access to water. Anaesthesia was induced with i.m. ketamine $30 \mathrm{mg} / \mathrm{kg}$, atropine $1 \mathrm{mg}$ and morphine $10 \mathrm{mg}$. A venous catheter was placed in the ear for infusion of Ringer acetate $30 \mathrm{ml} / \mathrm{kg} / \mathrm{h}$ and induction of anaesthesia with fentanyl 10 microgram $/ \mathrm{kg}$ and propofol $2 \mathrm{mg} / \mathrm{kg}$ i.v. Anaesthesia was maintained by infusion of fentanyl (3-10 microgram $/ \mathrm{kg} / \mathrm{min}$ ) and propofol $2-10 \mathrm{mg} / \mathrm{kg} / \mathrm{h}$ guided by hemodynamic response and need. The pig was intubated and ventilated with Datex-Ohmeda S5 ventilator $\left(\mathrm{FIO}_{2} 0.3\right.$, respiration rate (RR) $16 / \mathrm{min}$ and tidal volume (TV) $15 \mathrm{ml} / \mathrm{kg}$ ) targeted to expired end tidal carbon dioxide $\left(\mathrm{EtCO}_{2}\right)$ of $5.0 \pm 0.5 \mathrm{kPa}$ measured by Cosmo plus
(Novametrix Medical systems, Wallingford, CT USA). Mean arterial pressure (MAP) was maintained between 65 and $90 \mathrm{mmHg}$ with the use of Ringer Acetate if needed.

The pig was then placed on its back on a U-shaped bed and all limbs were fastened and the head fixated. The temperature was measured by a urine catheter placed via cystotomy and maintained at $38.0 \pm 0.5^{\circ} \mathrm{C}$ with the help of a heating/cooling mattress (Artic Sun, Medivance, Louisville, CO, USA).

Defibrillation pads placed in the upper right quadrant of the chest and lateral to columna on the left side of the chest were connected to a LIFEPAK 12 Monitor/ Defibrillator (Physio-Control, Redmond, WA, US).

The common and external carotid arteries were dissected. A Doppler flow meter probe (model 3SB880, Transonic Systems Inc., Ithaca, NY, USA) was placed on the right common carotid artery, and the external carotid artery was ligated. Two 7F micro-tip pressure transducer catheters (Model SPC 470, Millar Instruments, Houston, TX, USA) were placed, one through the right femoral artery up to the aortic arch and for continuous arterial pressures measurements $(\mathrm{SAP}=$ systolic aortic pressure, $\mathrm{MAP}=$ mean aortic pressure, DAP = diastolic aortic pressure), the second catheter was placed through the left external jugular vein to the right atrium for continuous pressure measurements $(\mathrm{SRAP}=$ systolic right atrial pressure, $\mathrm{MRAP}=$ mean right atrial pressure, $\mathrm{DRAP}=$ diastolic right atrial pressure). A 7.5F Swan-Ganz catheter (Edwards Lifesciences, Irvin, CA, USA) was placed in the pulmonary artery via the right femoral vein for thermodilution cardiac output and wedge pressure measurements. Another 7 . 5F Swan-Ganz catheter was placed in the right atrium through the left femoral vein, and a fluid filled polyethylene catheter was placed in aorta through the left femoral artery. These catheters were used for blood gases.

Oesophageal pressure was measured at the level of the heart using a cylindrical shaped rubber balloon (length $5 \mathrm{~cm}$, perimeter $3.4 \mathrm{~cm}$ ) containing air, glued to an open ended 7-F stiff catheter with multiple side holes, attached to a pressure transducer.

Craniotomy and duratomy were performed $10 \mathrm{~mm}$ anterior of the coronary suture and $15 \mathrm{~mm}$ to the left of the lateral part of the sagittal suture for a laser Doppler flowmeter probe (Modell 407, Perimed AB, Stockholm, Sweden) on the surface of cerebral cortex.

The skin over sternum was dissected and an oval shaped metal plate $(12 \times 6 \mathrm{~cm})$ secured to the sternum with 6-8 screws. A removable metal pin enabled fastening the modified LUCAS 2 device piston to the metal plate in order to achieve active decompression and pulling/lifting of the sternum when placed, and standard chest compressions when removed. The pin could be removed or inserted in 4-5 s. The LUCAS 2 
device used in present study was only physically modified by removing the suction cup, all other modification allowing ACD-CPR with $2 \mathrm{~cm}$ of additional decompression were accomplished by software alteration carried out by Physio-Control/Jolife AB, Lund, Sweden. Two $\mathrm{cm}$ of decompression was chosen because this amount of decompression combined with $5 \mathrm{~cm}$ of compression yielded best hemodynamic results in an earlier study with similar design [8].

\section{Monitored variables}

The following variables were monitored and continuously measured during the interventions: Systemic arterial pressure, right atrial pressure, intrathoracic pressure (oesophagus), cerebral blood flow (laser Doppler), carotid artery flow. Coronary perfusion pressure (CPP) was calculated as the difference between aortic pressure and right atrial pressure. In addition, the following variables were measured at specific time points during the experiment: cardiac output $(\mathrm{CO})$, arterial and central venous blood gases (ABG and CVBG), $\mathrm{EtCO}_{2}$ and bladder temperature.

All continuously measured pressure and flow signals were conditioned with Gould Transducer amplifiers in a Gould 6600 chassis (Gould Electronics) and sampled with a PC data acquisition system (NI SCXI-1000, NI PCI-6036E, National Instruments Company, Austin, TX, USA) with VI Logger software (National Instruments Company, Austin, TX, USA) and broken down to a sampling frequency of 100 per chest compression cycle.

\section{Experimental protocol (Fig. 1)}

We registered baseline measurements of pressures, flow, $\mathrm{CO}$ and $\mathrm{EtCO}_{2}$ after instrumentation and stabilization before induction of VF. A transcardial current (0.9 V DC) induced VF, which was verified by ECG and disappearance of pressures. At the same time anaesthesia, heating, i.v. fluids and ventilations were discontinued. No drugs were given during the three experimental phases. This noncirculatory state was continued for 2 min after VF induction. The chest wall was then «primed» for $30 \mathrm{~s}$. with the mechanical chest compression device with $3 \mathrm{~cm}$ compression depth and a frequency of $102 \pm 2 / \mathrm{min}$. This was done in order to adjust the base level for the chest compression depth due to initial changes in chest configuration caused by chest compressions. Phase 1 started after the chest was "primed". Pressures, flow and $\mathrm{EtCO}_{2}$ were measured continuously, cardiac output was measured at 60 and $150 \mathrm{~s}$ and blood gases (ABG, CVBG) $150 \mathrm{~s}$ into phase 1 . The pigs were manually ventilated $\left(\mathrm{FiO}_{2}\right.$ 1.0) by a person blinded for the $\mathrm{ETCO}_{2}$ value with a Laerdal bag connected to the endotracheal tube, 10-15 pr. min between chest compressions and a tidal volume of approximately $400-500 \mathrm{ml}$.

\section{Phase 1}

The piston was adjusted in order to touch the metal plate on the chest and the first $180 \mathrm{~s}$ phase of CPR was started. Mechanical chest compressions were delivered at a 50/50 compression/decompression phase duty cycle, a rate of $102 \pm 2 / \mathrm{min}$ and depth of $53 \mathrm{~mm} \pm$ $2 \mathrm{~mm}$ (control) or with the same depth and rate in addition to active decompression to $2 \mathrm{~cm}$ above normal anatomical chest level (intervention).

\section{Phase 2}

Active decompression was added or withdrawn based on what was performed in phase 1 .

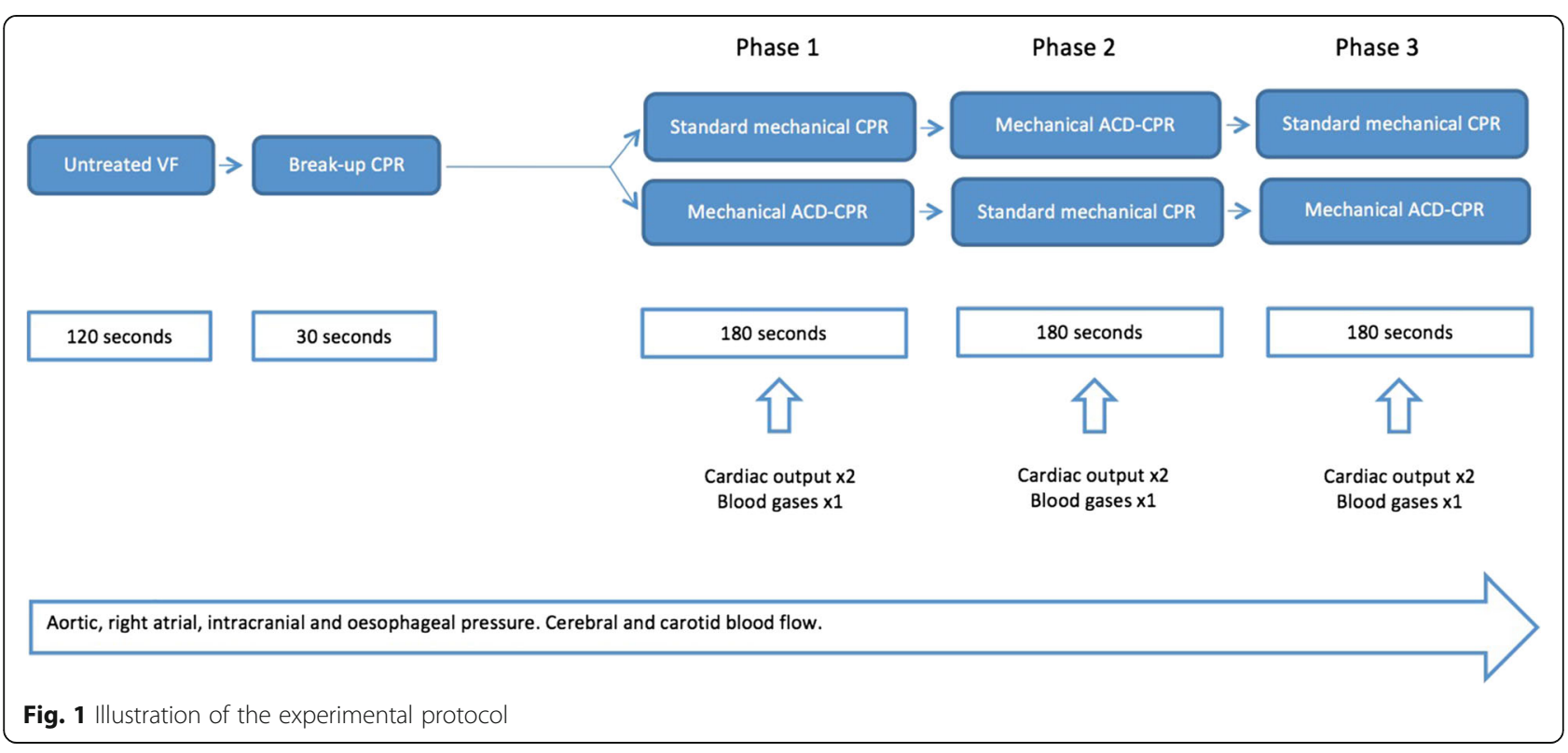




\section{Phase 3}

Active decompression was added or withdrawn again, and the same method (control or intervention) carried out as in phase 1.

After phase three the pigs received an overdose of propofol and $20 \mathrm{ml} 1 \mathrm{M} \mathrm{KCl}$, and CPR was continued for $1 \mathrm{~min}$. The experiment was finished when there was no pressure or flow generating cardiac activity. The chest wall and abdomen were opened in order to detect injuries and verify correct placement of the catheters.

\section{Power analysis}

We know from previous studies during optimally performed mechanical chest compressions (50/50 compression/decompression cycle, depth $4 \mathrm{~cm}$, rate 100/min) during VF in a porcine model that CPP is approximately 15-25 mmHg with standard deviation (SD) of 4 .

Power analysis performed in Sample Power shows that with crossover and paired analysis 10 pigs were needed in order to demonstrate a CPP difference of $10 \mathrm{mmHg}$ with the power of 0.99 and alfa 0.05 . The smallest difference in CPP to be documented with 10 pigs, power of 0 . 9 and SD 4, is $4.5 \mathrm{mmHg}$.

For the secondary endpoint carotid artery flow as percentage of basal flow, analysis indicated a power of 0.80 for a difference of 0.20 (absolute change in percentage flow) with SD 0.20 with 10 experiments.

\section{Statistical analysis}

We compared intervals during continuous mechanical chest compressions with or without active decompression. Two-sided paired samples t-test was used for continuous parametric data and Wilcoxon test for nonparametric data. $P<0.05$ was considered significant. Analyses were performed using IBM SPSS version 23/24 (IBM Corp. Armonk, NY, USA). Primary endpoint was $\mathrm{CPP}$ and secondary endpoints were cerebral blood flow and other haemodynamic parameters.

\section{Results}

\section{Haemodynamic results}

A total of 19 pigs $(34.0 \pm 3.3 \mathrm{~kg}, 20.2 \pm 0.9 \mathrm{~cm}$ AP chest diameter) were used in the present study, whereof 10 were excluded due to device failure or injury during the experiment. Among the nine included pigs, all aspects of the experimental protocol were concluded in eight. The experimental protocol had to be cancelled $45 \mathrm{~s}$ into phase 2 in one pig because of chest compression device failure. Data from both phase 1 and 2 in this pig were included in the analysis. The order of the three experimental phases was equally distributed among the remaining eight pigs. Descriptive characteristics and baseline values are presented in Table 1.
Table 1 Pig characteristic and pre-VF basal haemodynamic

\begin{tabular}{|c|c|}
\hline$N=9$ & $\begin{array}{l}\text { Mean } \pm \text { SD or } \\
\text { Median \& quartiles }\end{array}$ \\
\hline Weight (kg) & $34 \pm 3.3$ \\
\hline Anterior-Posterior chest-diameter $(\mathrm{cm})$ & $20 \pm 0.9$ \\
\hline Temperature (Celsius) & $38 \pm 0.6$ \\
\hline Cardiac Output (I/min) & $3.6(3.1,4.5)$ \\
\hline $\mathrm{EtCO}_{2}(\mathrm{kPa})$ & $5.4 \pm 0.8$ \\
\hline Aortic pressure $(\mathrm{mmHg})$ & $87 \pm 6.9$ \\
\hline Right atrial pressure $(\mathrm{mmHg})$ & $6.9(6.4,11)$ \\
\hline Coronary perfusion pressure $(\mathrm{mmHg})$ & $77(69,84)$ \\
\hline Intracranial pressure $(\mathrm{mmHg})$ & $14 \pm 4.4$ \\
\hline Oesophageal pressure $(\mathrm{mmHg})$ & $40 \pm 38$ \\
\hline Cerebral flow (AU) & $452 \pm 182$ \\
\hline Carotid flow (ml/min) & $181 \pm 18$ \\
\hline $\mathrm{pH}$ - Arterial blood gas & $7.4 \pm 0.2$ \\
\hline $\mathrm{pCO}_{2}$ - Arterial blood gas $(\mathrm{kPa})$ & $6.0 \pm 0.6$ \\
\hline $\mathrm{pO}_{2}$ - Arterial blood gas $(\mathrm{kPa})$ & $10.8 \pm 1.3$ \\
\hline $\mathrm{pH}$ - Venous blood gas & $7.3 \pm 0.02$ \\
\hline $\mathrm{pCO}_{2}-$ Venous blood gas $(\mathrm{kPa})$ & $7.3 \pm 0.8$ \\
\hline $\mathrm{pO}_{2}$ - Venous blood gas $(\mathrm{kPa})$ & $4.4 \pm 0.6$ \\
\hline
\end{tabular}

There were no significant differences in mean aortic, right atrial, oesophageal or intracranial pressures, $\mathrm{EtCO}_{2}$, arterial or venous blood gases between ACD-CPR and standard mechanical CPR. Cardiac output, cerebral and carotid blood flows were significantly higher during ACP-CPR (Table 2).

When analysing the different phases of the CPR cycle (Figs. 2, 3 and Table 3), aortic pressure was significantly higher in the peak compression phase during ACD-CPR. ICP was significantly lower in the end decompression phase during ACD-CPR. CPP trended towards higher values during $\mathrm{ACD}-\mathrm{CPR}$ in the late decompression phase $(p=0.06)$. Cerebral blood flow was significantly higher during ACD-CPR during all phases of the CPR cycle, while carotid artery flow did not show any significant differences in any specific CPR cycle phase.

\section{Injuries}

Experimental/device failure and injuries during instrumentation or early experimental phase warranted the additional use of ten pigs in order to finish the study. The reasons for exclusion were as follows: A sternum fixation screw punctured the heart $[n=1]$. Sternal fracture and puncture of the right atrium $[n=1]$. Loosening of sternal screws and plate $[n=2]$ or breaking of sternal plate $[n=1]$. Loss of cardiac output-values and/ 
Table 2 Comparison between standard mechanical CPR and ACD CPR

\begin{tabular}{|c|c|c|c|c|c|c|}
\hline & $\begin{array}{l}\text { Standard CPR } \\
\text { mean } \pm S D\end{array}$ & $\begin{array}{l}\text { ACD-CPR } \\
\text { mean } \pm S D\end{array}$ & $\begin{array}{l}\text { Mean difference } \\
(95 \% \mathrm{Cl})\end{array}$ & $\begin{array}{l}\text { Standard CPR } \\
\text { median } \\
\text { (quartiles) }\end{array}$ & $\begin{array}{l}\text { ACD-CPR } \\
\text { median } \\
\text { (quartiles) }\end{array}$ & $p$-value \\
\hline \multicolumn{7}{|l|}{ Blood pressures (mmHg) } \\
\hline Aortic pressure & & & & $55(51,70)$ & $60(51,70)$ & 0.86 \\
\hline Right atrial pressure & $54 \pm 16$ & $55 \pm 16$ & $1.0(-4.0,6.0)$ & & & 0.66 \\
\hline Coronary perfusion pressure & $2.2 \pm 22$ & $0.6 \pm 20$ & $-2.7(-10,4.7)$ & & & 0.42 \\
\hline Oesophageal pressure & $57 \pm 38$ & $60 \pm 39$ & $2.6(-2.6,7.8)$ & & & 0.28 \\
\hline Intracranial pressure & $24 \pm 6.4$ & $23 \pm 6.7$ & $-0.7(-1.6,0.1)$ & & & 0.09 \\
\hline \multicolumn{7}{|l|}{ Blood flow } \\
\hline Cerebral flow (AU) & $253 \pm 268$ & $297 \pm 264$ & $44(14,74)$ & & & 0.01 \\
\hline Carotid artery flow (ml/min) & & & & $83(57,94)$ & $97(70,106)$ & $<0.01$ \\
\hline \multicolumn{7}{|l|}{ Manual measurements } \\
\hline Cardiac output (I/min) & & & & $1.1(0.8,1.5)$ & $1.5(1.1,1.7)$ & $<0.01$ \\
\hline End tidal $\mathrm{CO}_{2}(\mathrm{kPa})$ & $2.2 \pm 0.8$ & $2.5 \pm 1.0$ & $0.3(-0.1,0.7)$ & & & 0.15 \\
\hline \multicolumn{7}{|l|}{ Blood gases (kPa) } \\
\hline $\mathrm{pH}$ - Arterial & & & & $7.3(7.2,7.4)$ & $7.3(7.2,7.4)$ & 0.33 \\
\hline $\mathrm{pCO}_{2}$ - Arterial & & & & $5.6(4.9,8.0)$ & $5.4(4.4,8.1)$ & 0.89 \\
\hline $\mathrm{pO}_{2}$ - Arterial & $22 \pm 20$ & $25 \pm 21$ & $2.5(-6.2,11)$ & & & 0.52 \\
\hline $\mathrm{pH}$ - Venous & & & & $7.1(7.1,7.2)$ & $7.2(7,1,7.2)$ & 0.09 \\
\hline $\mathrm{pCO}_{2}$ - Venous & $11 \pm 1.9$ & $11 \pm 1.9$ & $-0.3(-0.5,0.0)$ & & & 0.06 \\
\hline $\mathrm{pO}_{2}$ - Venous & & & & $3.1(3.0,3.6)$ & $3.3(3.1,3.6)$ & 0.11 \\
\hline
\end{tabular}

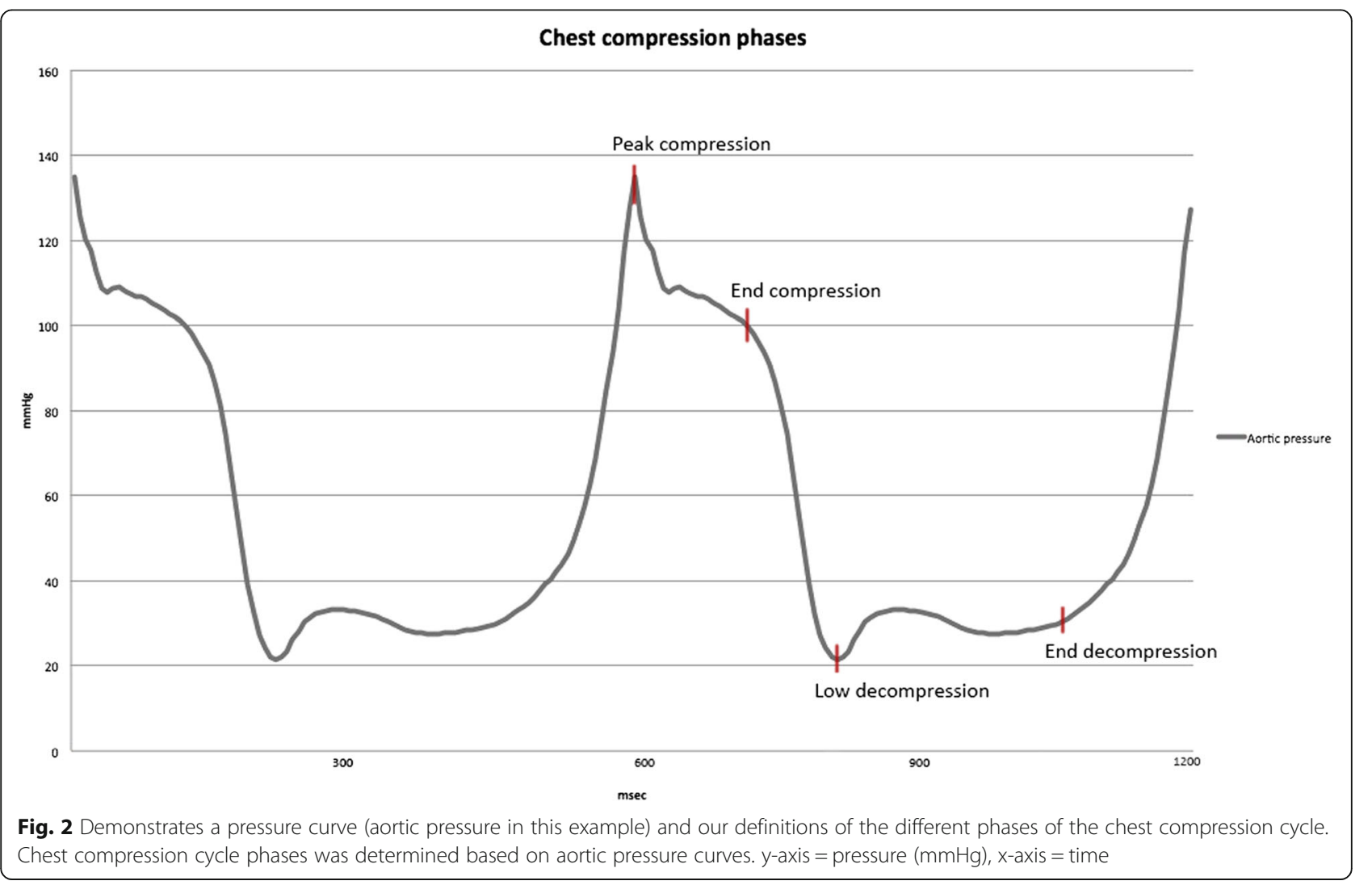




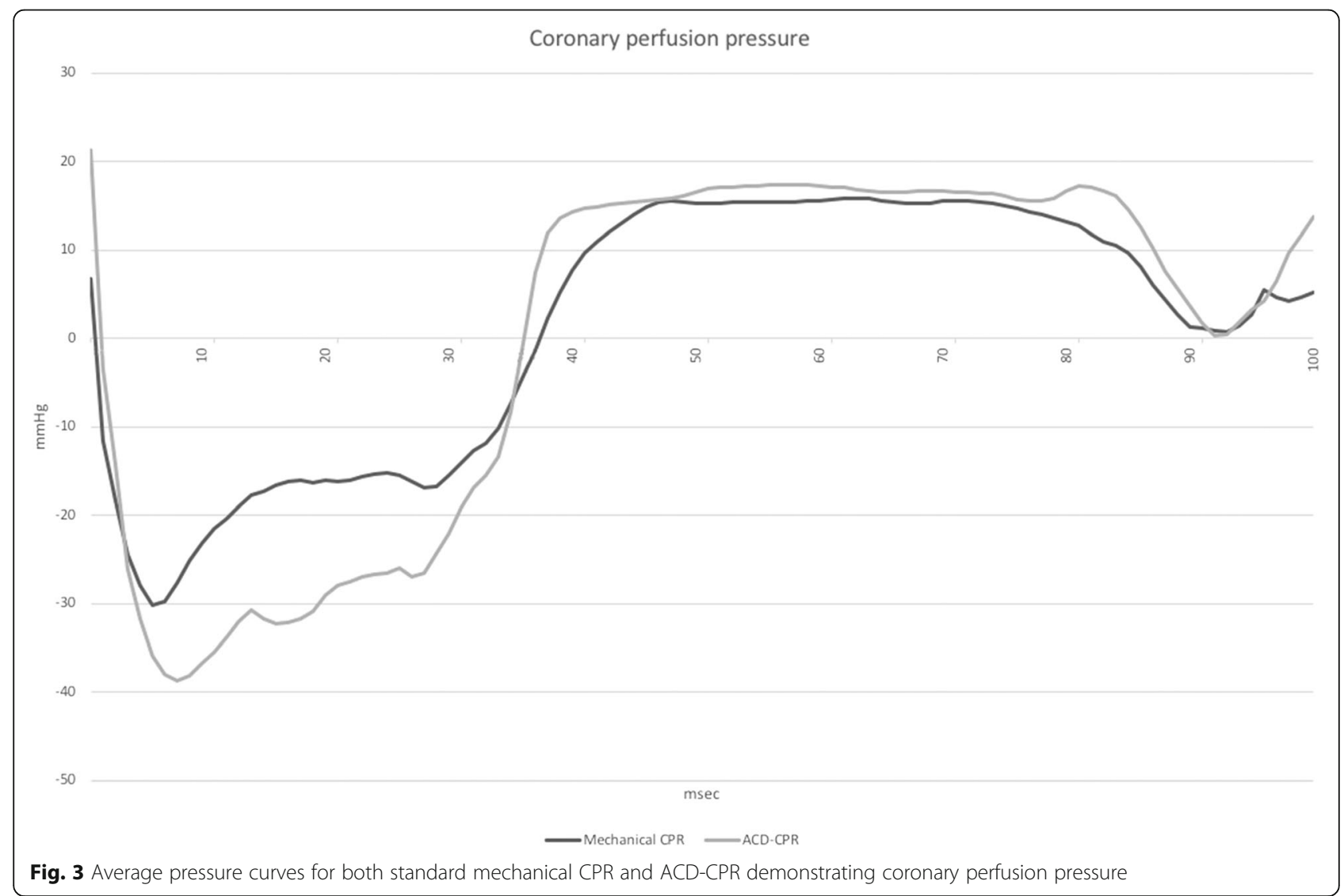

or large thoracic bleeding $[n=3]$. Compression device failure $[n=2]$.

\section{Discussion}

Our findings of increased cardiac output and cerebral blood flow during ACD-CPR are supported by earlier studies on dogs by Cohen et al. and Chang et al. $[9,12]$ As in most other studies they used a manual handheld device deliver ACD-CPR. Lindner et al. were the first to document higher cerebral blood flow during ACD-CPR when using piston based mechanical CPR in both $\mathrm{ACD}$ and control pigs [10].

No differences in mean pressures were demonstrated in present study, but aortic pressure was higher during peak compression with ACD-CPR, and CPP trended to be higher during end decompression with ACD-CPR (Fig. 2). Both these results are similar to what Wik et al. demonstrated in 1996 [8]. They also demonstrate lower oesophageal pressures during the end decompression phase with ACD-CPR, in addition to higher right atrial pressures during the peak and end compression phase of ACD-CPR. The present absolute values were similar, but we were unable to demonstrate significant differences. The data from Wik et al. were manually extracted based on printed pressure curves. Our data were collected by a real time data acquisition system and presented generally higher both aortic and right atrial pressures. Both studies found negative CPP values during what is equivalent to systolic parts of the chest compression cycle. This supports that CPP and thereby myocardial perfusion only takes place in the diastolic phase of mechanical ACDCPR. CPP is not a major determinant of myocardial blood flow within the physiological range of arterial blood pressure. Myocardial perfusion is however directly related to CPP in low-pressure scenarios such as CPR [24]. CPR-induced high intrathoracic pressure in the compression phase has low impact on myocardial perfusion because this pressure is also applied to the right side of the heart, thus not generating the arteriovenous pressure difference needed for coronary perfusion [25]. A higher aortic pressure in the decompression phase combined with a reduction in right atrial pressure is therefore the key to achieving increased myocardial perfusion during CPR. In the present study there was only a not significant trend $(p=0.06)$ towards higher CPP in the end decompression phase. We did not find an additional decrease in oesophageal pressure as a proxy for reduced intrathoracic pressure during ACD-CPR. One could speculate that the possibility for a further reduction in right atrial pressure could be achieved by combining ACD-CPR with an ITD, as demonstrated by Aufderheide et al. [23] Langhelle et al. also demonstrated a significant 
Table 3 Standard mechanical CPR compared with ACD CPR during different CPR cycle phases

\begin{tabular}{|c|c|c|c|c|c|c|}
\hline & $\begin{array}{l}\text { Standard CPR } \\
\text { mean } \pm S D\end{array}$ & $\begin{array}{l}\text { ACD-CPR } \\
\text { mean } \pm S D\end{array}$ & $\begin{array}{l}\text { Mean difference } \\
(95 \% \mathrm{Cl})\end{array}$ & $\begin{array}{l}\text { Standard CPR } \\
\text { median } \\
\text { (quartiles) }\end{array}$ & $\begin{array}{l}\text { ACD-CPR } \\
\text { median } \\
\text { (quartiles) }\end{array}$ & $p$-value \\
\hline \multicolumn{7}{|l|}{ Blood pressures (mmHg) } \\
\hline \multicolumn{7}{|l|}{ Aortic pressure } \\
\hline Peak compression & $180 \pm 78$ & $196 \pm 79$ & $16(1.1,30)$ & & & 0.04 \\
\hline End compression & $73 \pm 65$ & $68 \pm 85$ & $-4.9(-29,19)$ & & & 0.65 \\
\hline Low decompression & $10 \pm 11$ & $8.7 \pm 13$ & $-1.4(-8.6,5.8)$ & & & 0.66 \\
\hline End decompression & $28 \pm 10$ & $30 \pm 9.1$ & $2.0(-1.1,5.2)$ & & & 0.18 \\
\hline \multicolumn{7}{|l|}{ Right atrial pressure } \\
\hline Peak compression & $172 \pm 71$ & $180 \pm 75$ & $7.8(-17,33)$ & & & 0.49 \\
\hline End compression & $91 \pm 31$ & $97 \pm 32$ & $6.3(-3.2,16)$ & & & 0.16 \\
\hline Low decompression & & & & $7.7(5.5,22)$ & $13(5.6,21)$ & 0.59 \\
\hline End decompression & & & & $11(11,18)$ & $11(9.4,17)$ & 0.11 \\
\hline \multicolumn{7}{|c|}{ Coronary perfusion pressure } \\
\hline Peak compression & $8.3 \pm 39$ & $16 \pm 44$ & $7.8(-9.8,25)$ & & & 0.34 \\
\hline End compression & & & & $-21(-39,6.0)$ & $-19(-39,6.5)$ & 0.59 \\
\hline Low decompression & $-1.2 \pm 14$ & $-6.2 \pm 17$ & $-4.9(-11,1.4)$ & & & 0.11 \\
\hline End decompression & $12 \pm 11$ & $16 \pm 9.4$ & $4.3(-0.2,8.7)$ & & & 0.06 \\
\hline \multicolumn{7}{|l|}{ Intracranial pressure } \\
\hline Peak compression & & & & $38(28,44)$ & $41(24,45)$ & 0.37 \\
\hline End compression & $35 \pm 10$ & $35 \pm 12$ & $-0.2(-4.2,3.8)$ & & & 0.91 \\
\hline Low decompression & $23 \pm 8.3$ & $24 \pm 6.7$ & $1.4(-2.1,4.8)$ & & & 0.39 \\
\hline End decompression & $15 \pm 4.9$ & $14 \pm 5.2$ & $-0.7(-1.2,-0.1)$ & & & 0.03 \\
\hline \multicolumn{7}{|l|}{ Oesophageal pressure } \\
\hline Peak compression & $106 \pm 83$ & $118 \pm 94$ & $13(-17,42)$ & & & 0.35 \\
\hline End compression & $76 \pm 48$ & $84 \pm 51$ & $7.4(-3.3,18)$ & & & 0.15 \\
\hline Low decompression & $40 \pm 37$ & $40 \pm 34$ & $-0.4(-12,11)$ & & & 0.94 \\
\hline End decompression & $39 \pm 29$ & $39 \pm 32$ & $0.0(-7.8,7.7)$ & & & 0.99 \\
\hline \multicolumn{7}{|l|}{ Blood flow } \\
\hline \multicolumn{7}{|l|}{ Cerebral flow (AU) } \\
\hline Peak compression & $248 \pm 268$ & $285 \pm 261$ & $38(9.8,66)$ & & & 0.01 \\
\hline End compression & $244 \pm 255$ & $278 \pm 251$ & $34(8.7,60)$ & & & 0.02 \\
\hline Low decompression & $250 \pm 257$ & $288 \pm 254$ & $38(5.3,70)$ & & & 0.03 \\
\hline End decompression & $266 \pm 283$ & $316 \pm 283$ & $51(18,82)$ & & & $<0.01$ \\
\hline \multicolumn{7}{|c|}{ Carotid artery flow (ml/min) } \\
\hline Peak compression & $288 \pm 93$ & $311 \pm 106$ & $23(-16,62)$ & & & 0.21 \\
\hline End compression & & & & $159(59,321)$ & $176(100,223)$ & 0.17 \\
\hline Low decompression & & & & $7.7(-99,51)$ & $17(-47,97)$ & 0.37 \\
\hline End decompression & & & & $22(-19,98)$ & $38(-7.7,117)$ & 0.09 \\
\hline
\end{tabular}

decrease in right atrial pressure with ACD-CPR, both with and without ITD. The mean decrease was greater for ACD-CPR alone, but ACD-CPR and ITD combined resulted in a greater decrease in the early decompression phase. These differences were only statistical significant when compared with manual CPR, not when ACD with or without ITD were compared [26].

As already mentioned, both present and earlier studies indicate that the diastolic/decompression phase of the chest compression cycle is the period of coronary 
perfusion. Current and earlier papers have presented several points of measurement during the diastolic/decompression phase in order to demonstrate the change in pressure throughout the chest compression cycle [7, $8,26]$.

In addition to pressures we also measured flow during the different phases of the chest compression cycle. Our data shows higher mean cerebral blood flow during ACD-CPR, with significantly higher blood flow during all chest compression phases. Carotid artery flow did also show higher mean values in the ACD group, however no chest compression phase demonstrated significantly higher values. Similar hemodynamic benefits have been found in humans with ACD-CPR, but not improved short or long-term survival [13-17, 21]. In a similar study design Langhelle et al. did not demonstrate differences in brain blood flow for ACD-CPR with or without ITD vs. standard CPR. They found increased coronary flow for ACD-CPR both with and without an ITD vs. standard CPR, but no significant difference between ACD-CPR with vs. without ITD [26].

There were no differences in blood gases between ACD-CPR and standard mechanical CPR, and there was no hyperventilation with potential impact on cerebral blood flow.

All clinical studies included in earlier systematic reviews of ACD-CPR utilized a handheld suction-based device to deliver ACD-CPR [20-22]. This device is reported to require more energy than regular manual $\mathrm{CPR}$, and it has been documented that CPR quality suffers with significantly lower compression rate, depth and duration, in addition to inadequate decompression force compared to both regular manual and mechanical CPR $[18,19,27]$. This could partly explain why hemodynamic benefits of ACD-CPR in experimental studies failed to result in better clinical outcomes.

\section{Limitations}

The results from this experimental porcine study are not directly transferable to clinical cardiac arrest. A high number of pigs were excluded from the trial because of injuries and failure during instrumentation and the experimental phase. The mechanical chest compression device was fastened to the sternum with screws on the pigs in present study. The clinical version of the device uses a suction cup to adhere to the human chest. Injuries during instrumentation would therefore not be applicable to the clinical setting of cardiac arrest. Among the ten exclusions, four were related to sternal plate/ screw failure and two to machine failure. The remaining four pigs had severe injuries early in the experimental phase. These injuries were mainly due to rupture of large vessels in/out of the heart, heart tamponade, punctured right atrium, in addition to one episode of sternal fracture. We hypothesise that these injuries may have been a result of how the chest compression piston was fastened to a plate screwed directly on to the pig's sternum, resulting in a very direct transfer of forces. The pull on this plate would be especially large during the transition between compression and decompression, and a suction cup would absorb a lot of this energy. The combination of such large drag forces and the fact that a pig's pericardium adheres directly to the inside of the sternum could explain the injuries during the experimental phases of present study [28]. Future studies should take this into account and consider the use of a suction cup modified to a pig's chest. We have no way to analyse if there were significant differences in injuries between the ACD-CPR and standard CPR as each pig was its own control.

We cannot rule out a carry-over effect from control to intervention or vice versa because of the cross-over nature of the study design. The three-phase design does take this into account and should at least partly make this kind of bias less likely by repeating the first modality.

We cannot rule out that the surgical preparation of the pig with dissection of the carotid arteries and catheter placement through jugular veins could alter the blood flow entering and exiting the brain.

No studies have to our knowledge validated the use of Swan Ganz catheters for measuring cardiac output during cardiac arrest. They are widely used for measuring pulmonary pressure in both pulmonary, cardiac and resuscitation research. Correct placement of all catheters was confirmed after completion of the experiment by autopsy.

The experimental protocol included two minutes of untreated VF. This is shorter than in most clinical situations, particularly for unwitnessed cardiac arrest or when bystander CPR is initiated after telephone instructions. The results are therefore not directly transferable.

An ITD was not included in the study although such devices have demonstrated both haemodynamic and clinical benefits earlier. We wanted to study the effect of mechanical active decompression alone in order to study the outcome of one intervention at a time. Including an ITD could be a natural next step.

\section{Conclusion}

ACD-CPR delivered by a modified clinically used mechanical chest compression device with decompression to $2 \mathrm{~cm}$ above the resting level of the chest resulted in higher cardiac output, cerebral and carotid blood flow in addition to a trend towards higher enddiastolic CPP compared to standard mechanical chest compressions.

\section{Abbreviations}

ABG: Arterial blood gas; ACD-CPR: Active compression-decompression CPR; CO: Cardiac output; CPP: Coronary perfusion pressure; CPR: Cardiopulmonary 
resuscitation; CVBG: Central venous blood gas; DAP: Diastolic aortic pressure; DRAP: Diastolic right atrial pressure; $\mathrm{ETCO}_{2}$ : Expired end tidal carbon dioxide; ITD: Impedance threshold device; MAP: Mean aortic pressure; MRAP: Mean right atrial pressure; SAP: Systolic aortic pressure; SD: Standard deviation; SRAP: Systolic right atrial pressure; VF: Ventricular fibrillation

\section{Acknowledgements}

A special thank you to Petter Andreas Steen for his insightful help drafting and revising the manuscript.

\section{Funding}

The trial was funded by author's institutions, NAKOS and Institute of Experimental Medical Research, Oslo University Hospital. Olsen has received a research grant from Zoll Medical to NAKOS. Physio-Control Inc/Jolife provided the modified LUCAS device.

\section{Availability of data and materials}

The datasets used and analysed during the current study are available from the corresponding author on reasonable request.

\section{Authors' contributions}

MS contributed with data collection, data analysis and drafting of the manuscript. JAO contributed with data collection, data analysis and critical revision of the manuscript. ME contributed with data collection and critical revision of the manuscript. AN contributed with data collection and critical revision of the manuscript. PAN contributed with data collection, data analysis and critical revision of the manuscript. JK-J contributed with data collection and critical revision of the manuscript. BMH contributed with the modification of the LUCAS device, data collection and critical revision of the manuscript. LW contributed with idea and study design, data collection and critical revision of the manuscript. All authors read and approved the final manuscript

\section{Ethics approval and consent to participate}

The experiments were carried out in accordance with "Regulations on Animal Experimentation" under The Norwegian Animal Welfare Authority Act and approved by Norwegian Animal Research Authority (FOTS-ID 4931)

\section{Competing interests}

Steinberg, Kramer-Johansen, Neset, Eriksen \& Norseng have no conflicting interests. Wik is NAKOS rep. in medical advisory board Physio-Control, principal investigator for CIRC and LUCAS ACD study, patent holder of patents licensed to Zoll and Physio-Control. Hardig is employed by Physio-Control Inc./Jolife, the manufacturer and provider of the LUCAS device.

\section{Publisher's Note}

Springer Nature remains neutral with regard to jurisdictional claims in published maps and institutional affiliations.

\section{Author details}

${ }^{1}$ Institute of Clinical Medicine, University of Oslo, Oslo, Norway. ${ }^{2}$ Norwegian National Advisory Unit on Prehospital Emergency Medicine, Oslo University Hospital, Oslo, Norway. ${ }^{3}$ Department of Oncology, Oslo University Hospital, Oslo, Norway. ${ }^{4}$ Institute of Experimental Medical Research, Oslo University Hospital, Oslo, Norway. ${ }^{5}$ County Governor of Rogaland, Stavanger, Norway. ${ }^{6}$ Physio-Control/Jolife AB, Ideon Science Park, Lund, Sweden.

Received: 21 November 2017 Accepted: 10 April 2018 Published online: 24 April 2018

\section{References}

1. Halperin HR, Paradis N, Omato JP, Zviman M, Johnson J, Kern KB. Improved hemodynamics with a novel chest compression device in a porcine model of cardiac arrest. Circulation. 2002;106(19):538.

2. Ward KR, Menegazzi JJ, Zelenak RR, Sullivan RJ, McSwain NE Jr. A comparison of chest compressions between mechanical and manual CPR by monitoring end-tidal PCO2 during human cardiac arrest. Ann Emerg Med. 1993:22(4):669-74

3. McDonald JL. Systolic and mean arterial pressures during manual and mechanical CPR in humans. Ann Emerg Med. 1982;11(6):292-5.

4. Perkins GD, Lall R, Quinn T, Deakin CD, Cooke MW, Horton J, et al. Mechanical versus manual chest compression for out-of-hospital cardiac arrest (PARAMEDIC): a pragmatic, cluster randomised controlled trial. Lancet. 2014;385(9972):947-55.

5. Wik L, Olsen JA, Persse D, Sterz F, Lozano M Jr, Brouwer MA, et al. Manual vs. integrated automatic load-distributing band CPR with equal survival after out of hospital cardiac arrest. The randomized CIRC trial. Resuscitation. 2014; 85(6):741-8.

6. Rubertsson S, Lindgren E, Smekal D, Ostlund O, Silfverstolpe J, Lichtveld RA, et al. Mechanical chest compressions and simultaneous defibrillation vs conventional cardiopulmonary resuscitation in out-of-hospital cardiac arrest: the LINC randomized trial. JAMA. 2014;311(1):53-61.

7. Wik L, Naess PA, llebekk A, Steen PA. Simultaneous active compressiondecompression and abdominal binding increase carotid blood flow additively during cardiopulmonary resuscitation (CPR) in pigs. Resuscitation. 1994;28(1):55-64

8. Wik L, Naess PA, llebekk A, Nicolaysen G, Steen PA. Effects of various degrees of compression and active decompression on haemodynamics, end-tidal $\mathrm{CO} 2$, and ventilation during cardiopulmonary resuscitation of pigs. Resuscitation. 1996;31(1):45-57.

9. Cohen TJ, Tucker KJ, Redberg RF, Lurie KG, Chin MC, Dutton JP, et al. Active compression-decompression resuscitation: a novel method of cardiopulmonary resuscitation. Am Heart J. 1992;124(5):1145-50.

10. Lindner $\mathrm{KH}$, Pfenninger EG, Lurie KG, Schurmann W, Lindner IM, Ahnefeld FW. Effects of active compression-decompression resuscitation on myocardial and cerebral blood flow in pigs. Circulation. 1993;88(3): 1254-63.

11. Tucker K, Khan JH, Savitt MA. Active compression-decompression resuscitation: effects on pulmonary ventilation. Resuscitation. 1993;26(2):125-31.

12. Chang MW, Coffeen P, Lurie KG, Shultz J, Bache RJ, White CW. Active compression-decompression CPR improves vital organ perfusion in a dog model of ventricular fibrillation. Chest. 1994:106(4):1250-9.

13. Cohen TJ, Tucker KJ, Lurie KG, Redberg RF, Dutton JP, Dwyer KA, et al. Active compression-decompression. A new method of cardiopulmonary resuscitation. Cardiopulmonary resuscitation working group. JAMA. 1992; 267(21):2916-23.

14. Guly UM, Robertson CE. Active decompression improves the haemodynamic state during cardiopulmonary resuscitation. Br Heart J. 1995; 73(4):372-6.

15. Tucker KJ, Redberg RF, Schiller NB, Cohen TJ. Active compressiondecompression resuscitation: analysis of transmitral flow and left ventricular volume by transesophageal echocardiography in humans. Cardiopulmonary resuscitation working group. J Am Coll Cardiol. 1993;22(5):1485-93.

16. Shultz JJ, Coffeen P, Sweeney M, Detloff B, Kehler C, Pineda E, et al. Evaluation of standard and active compression-decompression CPR in an acute human model of ventricular fibrillation. Circulation. 1994;89(2): 684-93

17. Orliaguet GA, Carli PA, Rozenberg A, Janniere D, Sauval P, Delpech P. Endtidal carbon dioxide during out-of-hospital cardiac arrest resuscitation: comparison of active compression-decompression and standard CPR. Ann Emerg Med. 1995;25(1):48-51.

18. Skogvoll E, Wik L. Active compression-decompression cardiopulmonary resuscitation (ACD-CPR) compared with standard CPR in a manikin modeldecompression force, compression rate, depth and duration. Resuscitation. 1997;34(1):11-6.

19. Sunde K, Wik L, Steen PA. Quality of mechanical, manual standard and active compression-decompression CPR on the arrest site and during transport in a manikin model. Resuscitation. 1997;34(3):235-42.

20. Wang $\mathrm{CH}$, Tsai MS, Chang WT, Huang $\mathrm{CH}, \mathrm{Ma} \mathrm{MH}$, Chen WJ, et al. Active compression-decompression resuscitation and impedance threshold device for out-of-hospital cardiac arrest: a systematic review and metaanalysis of randomized controlled trials. Crit Care Med. 2015;43(4):889-96.

21. Lafuente-Lafuente C, Melero-Bascones M. Active chest compressiondecompression for cardiopulmonary resuscitation. Cochrane Database Syst Rev. 2013;(9):1465-1858.

22. Cabrini L, Beccaria P, Landoni G, Biondi-Zoccai GG, Sheiban I, Cristofolini M et al. Impact of impedance threshold devices on cardiopulmonary resuscitation: a systematic review and meta-analysis of randomized controlled studies. Crit Care Med. 2008;36(5):1625-32.

23. Aufderheide TP, Frascone RJ, Wayne MA, Mahoney BD, Swor RA, Domeier RM, et al. Comparative effectiveness of standard CPR versus active compression decompression CPR with augmentation of negative intrathoracic pressure for treatment of out-of-hospital cardiac arrest: 
results from a randomized prospective study. Lancet. 2011;377(9762): $301-11$.

24. Mosher P, Ross J Jr, McFate PA, Shaw RF. Control of coronary blood flow by an autoregulatory mechanism. Circ Res. 1964;14:250-9.

25. Ditchey RV, Winkler JV, Rhodes CA. Relative lack of coronary blood flow during closed-chest resuscitation in dogs. Circulation. 1982;66(2):297-302.

26. Langhelle A, Stromme T, Sunde K, Wik L, Nicolaysen G, Steen PA. Inspiratory impedance threshold valve during CPR. Resuscitation. 2002;52(1):39-48.

27. Shultz JJ, Mianulli MJ, Gisch TM, Coffeen PR, Haidet GC, Lurie KG. Comparison of exertion required to perform standard and active compression-decompression cardiopulmonary resuscitation. Resuscitation. 1995;29(1):23-31.

28. R N. Lehrbuch der Anatomie der Haustiere Band III: Kreislaufsystem, Haut und Hautorgane: Verlag Paul Parey; 1976. p. 17-18.

Ready to submit your research? Choose BMC and benefit from:

- fast, convenient online submission

- thorough peer review by experienced researchers in your field

- rapid publication on acceptance

- support for research data, including large and complex data types

- gold Open Access which fosters wider collaboration and increased citations

- maximum visibility for your research: over 100M website views per year

At BMC, research is always in progress.

Learn more biomedcentral.com/submissions 